JOURNAL OF THE

AMERICAN MATHEMATICAL SOCIETY

Volume 15, Number 3, Pages 715-786

S 0894-0347(02)00389-2

Article electronically published on April 5, 2002

\title{
CONSTRUCTION OF DISCRETE SERIES FOR CLASSICAL $p$-ADIC GROUPS
}

\author{
COLETTE MCEGLIN AND MARKO TADIĆ
}

\section{INTRODUCTION}

The goal of this paper is to complete (after [M2]) the classification of irreducible square integrable representations of classical $p$-adic groups, under a natural local assumption (see below). This classification (see Theorem 6.1) also implies a parameterization of irreducible tempered representations of these groups (Theorem 13.1). Therefore, it implies a classification of the non-unitary duals of these groups (modulo cuspidal data).

The classical groups whose classification of irreducible square integrable representations we give are symplectic, orthogonal and unitary groups over a nonarchimedean local field $F$. For simplicity, in this introduction we shall explain the classification in the case of symplectic and odd-orthogonal groups (in the case of unitary groups, the Galois interpretation of the classification is substantially more complicated). Denote by $G$ a symplectic or odd-orthogonal group on the space (of the corresponding type) of dimension $2 n$ or $2 n+1$ respectively.

The classification of irreducible square integrable representations is directly related to the parameterization of irreducible square integrable representations in terms of dual objects, which is predicted by Langlands program. These dual objects consist of two parts. The first part is a semi-simple morphism

$$
\varphi: W_{F} \times S L(2, \mathbb{C}) \rightarrow{ }^{L} G,
$$

where ${ }^{L} G$ is the dual group of $G$ (in the case that we consider, even for a non-split orthogonal group, we can replace ${ }^{L} G$ by its connected component; see [M3]). The morphism $\varphi$ has to be algebraic and discrete (by discrete we mean that it does not factor through a proper Levi subgroup). The second part is a morphism

$$
\epsilon: \mathrm{Cent}_{L_{G}}(\operatorname{Im}(\varphi)) \rightarrow\{ \pm 1\}
$$

with the following restriction to the center of ${ }^{L} G$ if $G$ is odd-orthogonal: the restriction of $\epsilon$ to the center of ${ }^{L} G$ is trivial if the anisotropic kernel has dimension 1 , and is -1 if it has dimension 3 . In fact, to take complete care of the anisotropic kernel, we need to require a condition on $\operatorname{det}(\varphi)$, which is not now important to us. This is explained in [M3] for the case of even-orthogonal groups. The condition on

Received by the editors December 1, 2000 and, in revised form, January 2, 2002.

1991 Mathematics Subject Classification. Primary 22E50, 22E35; Secondary 11F70, 11S37.

Key words and phrases. Classical groups, $p$-adic fields, irreducible square integrable representations, irreducible tempered representations, non-unitary dual, local Langlands correspondences.

The second author was partly supported by Croatian Ministry of Science and Technology grant \# 37001. 
$\epsilon$ and $\operatorname{det}(\varphi)$ enables us to avoid requiring in the definition of being discrete that the Levi factors of ${ }^{L} G$ are defined over $F$ (we shall assume that $\varphi$ and $\epsilon$ satisfy the above requirements).

A simple form of the parameterization of irreducible square integrable representations predicted by Langlands program would be a bijection between sets of all (isomorphism classes of) irreducible square integrable representations of $G$ and conjugacy classes of pairs

$$
(\varphi, \epsilon)
$$

as above. This parameterization needs to satisfy a number of properties to be unique (these properties concern liftings and the local harmonic analysis). Our elementary method does not give information in that direction. Further, although our classification is on the line of the parameterization of irreducible square integrable representations predicted by Langlands program, it does not prove that such a simple form of the parameterization of irreducible square integrable representations exists. The problem is caused by the cuspidal representations, as we shall explain later. A pair $(\varphi, \epsilon)$, which should correspond to a cuspidal representation, should have a description in terms of cuspidal sheaves à la Lusztig. In our case, we can give a much more elementary description of such a pair.

First of all, it is easy to describe $\operatorname{Cent}_{L_{G}}(\operatorname{Im}(\varphi))$, especially in the discrete case. Consider the decomposition of $\varphi$ into irreducible components:

$$
\varphi=\bigoplus_{(\rho, a)} \rho \otimes E_{a} .
$$

In the above direct sum, $\rho$ are irreducible representations of $W_{F}$ (necessarily orthogonal or symplectic, which follows from the property that $\varphi$ is discrete) and $E_{a}$ is the irreducible (complex) algebraic representation of $S L(2, \mathbb{C})$ of dimension $a \in \mathbb{N}$. This decomposition must be multiplicity free (this follows from the property that $\varphi$ is discrete). The parity of $a$ is uniquely determined by $\rho$ and $G$. Denote by

$$
\operatorname{Jord}(\varphi)
$$

the set of all indexes $(\rho, a)$ in the above direct sum decomposition. In particular, for $(\rho, a) \in \operatorname{Jord}(\varphi), \rho \otimes E_{a}$ factors through an orthogonal subgroup of ${ }^{L} G$ if $G$ is symplectic, and through a symplectic subgroup if $G$ is orthogonal. In both cases, this subgroup has a center isomorphic to $\mathbb{Z} / 2 \mathbb{Z}$, and further

$$
\operatorname{Cent}_{L_{G}}(\operatorname{Im}(\varphi)) \simeq \prod_{(\rho, a) \in \operatorname{Jord}(\varphi)}(\mathbb{Z} / 2 \mathbb{Z}),
$$

where one needs to take elements of determinant one if $G$ is symplectic (in the orthogonal case, we have no restrictions).

Now we shall consider certain mappings

$$
\epsilon: \operatorname{Jord}(\varphi) \rightarrow\{ \pm 1\}
$$

(they correspond to morphisms of $\operatorname{Cent}_{L_{G}}(\operatorname{Im}(\varphi))$ into $\left.\{ \pm 1\}\right)$. It is convenient to define

$\operatorname{Jord}^{+}(\varphi):=\operatorname{Jord}(\varphi) \cup\{(\rho, 0)$; there exists $a \in 2 \mathbb{N}$ such that $(\rho, a) \in \operatorname{Jord}(\varphi)\}$.

We shall extend $\epsilon$ as above to $\epsilon^{+}$on $\operatorname{Jord}^{+}(\varphi)$ by defining $\epsilon^{+}(\rho, 0)=1$. We say that $(\varphi, \epsilon)$ is cuspidal if and only if, for any $(\rho, a) \in \operatorname{Jord}^{+}(\varphi)$ such that $a \geq 2$, we 
have $(\rho, a-2) \in \operatorname{Jord}^{+}(\varphi)$ and

$$
\epsilon(\rho, a) \neq \epsilon^{+}(\rho, a-2) .
$$

Cuspidal pairs should correspond to cuspidal representations. Let us note that in the cuspidal case we do not have a lot of evidence that the set of all equivalence classes of irreducible cuspidal representations is in a bijection with the set of all conjugacy classes of cuspidal pairs $(\varphi, \epsilon)$. The case which is well understood and which gives the evidence is the case where all $\rho$ in $\operatorname{Jord}(\varphi)$ are quadratic characters (see [M3]). Besides this, we can expect progress in the level 0 case, which would provide further evidence.

To avoid a hypothesis regarding the cuspidal case, we proceed in the following way. On the side of the irreducible representations of $G$, we have the notion of the cuspidal support. A weaker notion of the cuspidal support is the notion of a partial cuspidal support. The definition is the following. Let $\pi$ be an irreducible representation of $G$. Denote by $\pi_{c u s p}$ an irreducible cuspidal representation of a subgroup $G^{\prime}$ of $G$ from the same series of groups (symplectic or odd-orthogonal), which satisfies the following condition: there exist a non-negative integer $k$ and an irreducible representation $\tau$ of $G L(k, F)$ such that $G L(k, F) \times G^{\prime}$ is (isomorphic to) a Levi factor of a (standard) parabolic subgroup $P$ of $G$ and $\pi$ is (isomorphic to) a subquotient of the parabolically induced representation $\tau \rtimes \pi_{c u s p}=\operatorname{Ind}_{P}^{G}\left(\tau \otimes \pi_{\text {cusp }}\right)$. Such $\pi_{\text {cusp }}$ always exists, it is unique (up to an isomorphism) and it is called the partial cuspidal support of $\pi$.

The partial cuspidal support has an analogue on the Galois side, for the pair $(\varphi, \epsilon)$. A combinatorial exercise (see section 14) implies that for each fixed pair $(\varphi, \epsilon)$ as above, there exists a unique cuspidal pair $\left(\varphi_{\varphi, \epsilon, c u s p}, \epsilon_{\varphi, \epsilon, c u s p}\right)$ (called the cuspidal support of $(\varphi, \epsilon))$ corresponding to some subgroup $G^{\prime}$ of $G$ as above, which satisfies one of the following two conditions (these conditions are related to two possible types of $(\varphi, \epsilon))$ :

(1) We shall say that a pair $(\varphi, \epsilon)$ is alternated if $\epsilon\left(\rho, a_{-}\right) \neq \epsilon(\rho, a)$ for each pair $\left(\rho, a_{-}\right),(\rho, a) \in \operatorname{Jord}(\varphi)$ which satisfies: $a_{-}<a$ and $(\rho, b) \notin \operatorname{Jord}(\varphi)$ for any $a_{-}<b<a$. If $(\varphi, \epsilon)$ is alternated, the cuspidal support $\left(\varphi_{\varphi, \epsilon, c u s p}, \epsilon_{\varphi, \epsilon, c u s p}\right)$ must satisfy the following: There exists an injection

$$
\psi: \operatorname{Jord}(\varphi) \rightarrow \operatorname{Jord}^{+}\left(\varphi_{\varphi, \epsilon, \text { cusp }}\right)
$$

whose image contains $\operatorname{Jord}\left(\varphi_{\varphi, \epsilon, c u s p}\right)$. Further, for any irreducible representation $\rho$ such $(\rho, b) \in \operatorname{Jord}(\varphi)$ for some positive integer $b$, there needs to exist a monotone mapping $\psi_{\rho}$ (i.e. preserves the ordering of $\mathbb{Z}_{+}$) such that

$$
\psi(\rho, a)=\left(\rho, \psi_{\rho}(a)\right)
$$

and

$$
\epsilon(\rho, a)=\epsilon_{\varphi, \epsilon, \text { cusp }}\left(\rho, \psi_{\rho}(a)\right)
$$

for any $(\rho, a) \in \operatorname{Jord}(\varphi)$.

Let us note that the condition in the definition of the cuspidal pair determines what is the image of $\psi$.

(2) Suppose that $(\varphi, \epsilon)$ is not alternated. Then there exist $\left(\rho, a_{-}\right)$and $(\rho, a) \in$ $\operatorname{Jord}(\varphi), a_{-}<a$, which satisfy the following properties:

$$
\text { if } a_{-}<b<a \text {, then }(\rho, b) \notin \operatorname{Jord}(\varphi)
$$


and

$$
\epsilon\left(\rho, a_{-}\right)=\epsilon(\rho, a) .
$$

Define

$$
\varphi_{1}:=\bigoplus_{\left(\rho^{\prime}, a^{\prime}\right) \in \operatorname{Jord}(\varphi) \backslash\left\{\left(\rho, a_{-}\right),(\rho, a)\right\}} \rho^{\prime} \otimes E_{a^{\prime}} .
$$

This is a parameter for a subgroup $G_{1}$ of $G$ of the same type. There is an obvious way to define a restriction $\epsilon_{1}$ of $\epsilon$ corresponding to the group $G_{1}$. Applying this construction several times, we shall come from $(\varphi, \epsilon)$ in finitely many steps to some $\left(\varphi^{\prime}, \epsilon^{\prime}\right)$ which is of alternated type. We define $\left(\varphi_{\varphi, \epsilon, \text { cusp }}, \epsilon_{\varphi, \epsilon, \text { cusp }}\right)$ to be the cuspidal support of $\left(\varphi^{\prime}, \epsilon^{\prime}\right)$ (as defined in (1)).

The definition of $\varphi_{\varphi, \epsilon, \text { cusp }}$ depends on both $\varphi$ and $\epsilon$. The fibers of the mapping $(\varphi, \epsilon) \mapsto\left(\varphi_{\varphi, \epsilon, \text { cusp }}, \epsilon_{\varphi, \epsilon, \text { cusp }}\right)$ have the following simple property. To any $(\varphi, \epsilon)$, we can associate $\left(\varphi, \Delta_{\epsilon}\right)$, where $\Delta_{\epsilon}$ is the morphism

$$
\operatorname{Jord}^{+}(\varphi) \times \operatorname{Jord}^{+}(\varphi) \rightarrow\{ \pm 1\},
$$

defined only on the pairs $(\rho, a),\left(\rho^{\prime}, a^{\prime}\right)$ for which $\rho=\rho^{\prime}$. On such a pair, $\Delta_{\epsilon}$ is defined by

$$
\Delta_{\epsilon}\left((\rho, a),\left(\rho, a^{\prime}\right)\right)=\epsilon(\rho, a) \epsilon\left(\rho, a^{\prime}\right)^{-1} .
$$

An easy combinatorial exercise (see section 14) shows that the fibers of the mapping

$$
(\varphi, \epsilon) \mapsto\left(\varphi, \Delta_{\epsilon}\right)
$$

are subsets of the fibers of the mapping

$$
(\varphi, \epsilon) \mapsto \varphi_{\varphi, \epsilon, \text { cusp }}
$$

Now we can explain our classification of (equivalence classes of) irreducible square integrable representations of $G$. Recall that in M2 to each irreducible square integrable representation $\pi$ of $G$ is associated (using elementary techniques) a set $\operatorname{Jord}(\pi)$ of a similar type as $\operatorname{Jord}(\varphi)$ (see section 2, where we recall the definition). We use the local Langlands correspondence for general linear groups to identify these two type of sets $(\operatorname{Jord}(\pi)$ 's and $\operatorname{Jord}(\varphi)$ 's). There is still a problem with the parity: parity of $a$ for $(\rho, a) \in \operatorname{Jord}(\varphi)$ depends on $G$, and on whether $\rho$ is orthogonal or symplectic. On the other side, the parity of $a$ for $(\rho, a) \in \operatorname{Jord}(\pi)$ depends on $G$, and on the pole of a certain $L$-function. Conjecturally, the pole (or holomorphy) reflects exactly the orthogonality or the symplecticity property. This is true on the Galois side, but it is not yet proved that the Langlands correspondence preserves this property (G. Henniart has recently announced a proof). In this introduction, we shall assume this property. In the rest of the paper, we shall not use the $L$-group explicitly. Therefore we do not (need to) assume this. In [M2] a mapping $\Delta_{\pi}$ which is of the same type as the $\Delta_{\epsilon}$ 's considered above is associated to $\pi$. Actually, in [M2] the notation $\epsilon_{\pi}$ is used instead of $\Delta_{\pi}$. It would be more consistent if $\Delta_{\pi}$ was used there (see section 2, the part following (2-1), for the definition of $\epsilon_{\pi}$ which corresponds to $\Delta_{\pi}$ in the present paper). With our elementary techniques, we are not able to prove the dimension relation:

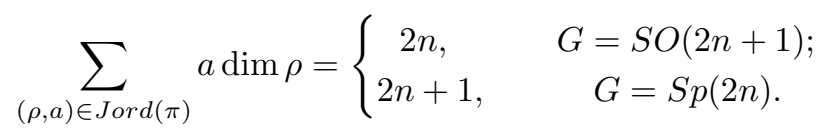


We do not use this equality in the paper, except here in the introduction. We will only assume the inequality $\leq$ which is now known by [M5. This is the easier of two inequalities, which would give the equality ( $\geq$ is the difficult part). Let us note that this dimension property can be proved if we assume that some Arthur's conjectures hold. Therefore, we can (and it makes sense to) take this dimension equality as a hypothesis (but only in the introduction).

Let us now state the principal result of [M2]. Assume that the basic assumption (which will be discussed later) holds. Then the mapping

$$
\pi \mapsto\left(\pi_{\text {cusp }}, \operatorname{Jord}(\pi), \Delta_{\pi}\right),
$$

which is defined on the set of all equivalence classes of irreducible square integrable representations of $G$, is injective, and it has the property that

$$
\operatorname{Jord}\left(\pi_{\text {cusp }}\right)=\operatorname{Jord}\left(\varphi_{\varphi, \epsilon, \text { cusp }}\right)
$$

for any $(\varphi, \epsilon)$ such that $\operatorname{Jord}(\varphi)=\operatorname{Jord}(\pi)$ and $\Delta_{\epsilon}=\Delta_{\pi}$ (we use the identification of $\operatorname{Jord}(\varphi)$ 's and $\operatorname{Jord}(\pi)$ 's resulting from the local Langlands correspondences for general linear groups). The last assertion is the admissibility condition from the introduction of [M2].

The aim of this paper is to prove that the mapping $\pi \mapsto\left(\pi_{\text {cusp }}, \operatorname{Jord}(\pi), \Delta_{\pi}\right)$ given above is surjective. The main consequence of this proof is the fact that the equivalence classes of irreducible square integrable representations of $G$ are classified by the triples

$$
\left(\pi_{\text {cusp }}, \varphi, \Delta_{\epsilon}\right) .
$$

In such a triple, $\pi_{\text {cusp }}$ is a cuspidal representation of a subgroup of $G$ from the same series of groups (symplectic or orthogonal). Further, $\varphi$ and $\Delta_{\epsilon}$ are coming in the way that we have explained above from a pair $\varphi, \epsilon$ which satisfies

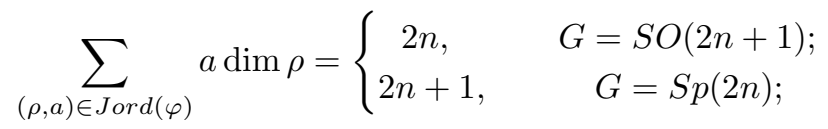

and

$$
\operatorname{Jord}\left(\pi_{\text {cusp }}\right)=\operatorname{Jord}\left(\varphi_{\varphi, \epsilon, \text { cusp }}\right) .
$$

The property that $\varphi$ is discrete has a simple translation: $\operatorname{Jord}(\varphi)$ is multiplicity free.

The surjectivity is proved under the basic assumption (BA) (see the second section), which describes the reducibility points of a representation induced by an irreducible cuspidal representation in terms of the Jordan bloc. This basic assumption was supposed to hold in [M2. In [M1] it is proved that (BA) follows from a weak form of Arthur's conjectures (they are recalled in [M1]). Note that here we do not use any global work and that (BA) is purely a local statement. Our basic assumption provides us with a possibility to compute Plancherel measures (modulo holomorphic invertible functions) in terms of Jordan blocs. This point of view was already present in [Sh1]. There F. Shahidi proved (BA) for generic cuspidal representations. Therefore, if $\pi_{\text {cusp }}$ is generic, we do not need to assume in our paper that (BA) holds (i.e. our paper has no hypothesis if $\pi_{c u s p}$ is generic).

In fact, in this paper we avoid the use of the dual group in order to minimize the assumptions on which this paper relies. We proceed in this paper much more technically (and more directly). As explained above, our classification starts from 
cuspidal representations. We classify all irreducible square integrable subquotients of the (generalized) principal series (i.e. all non-cuspidal irreducible square integrable representations). A generalized principal series is an induced representation:

$$
\left(\prod_{k=1}^{\ell} \nu^{r_{k}} \rho_{k}\right) \rtimes \pi_{\text {cusp }}
$$

where $\ell \geq 0, \rho_{k}$ are irreducible unitarizable representations of a general linear group, $\nu$ is $|\operatorname{det}|_{F}$ and $r_{k} \in \mathbb{R}$. Further, $\pi_{\text {cusp }}$ is an irreducible cuspidal representation of some subgroup $G^{\prime}$ of $G$ from the same series of groups (symplectic or orthogonal; see the first section for details regarding the notation). We do the classification using only a (natural) assumption about reducibility points of parabolically induced representations of the following type:

$$
\nu^{x} \rho_{k} \rtimes \pi_{c u s p},
$$

where $k \in \mathcal{K}$ and $x \in \mathbb{R}$. Our basic assumption is that this induced representation is irreducible except when one of the following two possibilities occur:

(i) $x=0$ or $\pm 1 / 2,\left(\rho_{k}, a\right) \notin \operatorname{Jord}\left(\pi_{\text {cusp }}\right)$ for all $a \in \mathbb{N}$ and $\nu^{x} \rho_{k} \rtimes 1$ reduces (here we assume for simplicity that the group is split);

(ii) $|x| \geq 1$ and $\left(\rho_{k}, 2|x|-1\right) \in \operatorname{Jord}\left(\pi_{\text {cusp }}\right)$ but $\left(\rho_{k}, 2|x|+1\right) \notin \operatorname{Jord}\left(\pi_{\text {cusp }}\right)$

(see section 2, and also section 12 for another interpretation; see also section 13 of [T2 for supporting evidence regarding (ii)). Moreover, we assume that a reducibility point as above, for fixed $\rho_{k}$, is unique up to a sign. G. Muić and F. Shahidi have explained that this uniqueness follows from the work of A. Silberger ([Si]).

We shall now briefly describe the content of the paper. First, let us recall that in the paper we shall never use the $L$-group explicitly. Thus, there are no morphisms $\varphi$ in the paper. It is replaced by its Jordan blocs, i.e. by a set Jord satisfying a parity condition (and a dimension condition). This explains why $\varphi$ 's disappear in the rest of the paper. There is also a significant difference between the use of the notation $\epsilon$ in this introduction, and the use of this notation in the rest of the paper. In the rest of the paper, except for the introduction, $\epsilon$ will denote the partial function which we have denoted by $\Delta_{\epsilon}$ in this introduction. Nevertheless, in our description of the content of the paper which follows, we continue with the notation which we have used above.

The first section introduces the notation. To simplify the exposition of the paper, after introducing notation for classical groups, we restrict ourselves until the end of section 14 to the case of symplectic and odd-orthogonal groups. The necessary modifications and comments regarding unitary and even-orthogonal groups are given in sections 15 and 16. The second section recalls in detail the basic assumption and the admissible triples. The following three sections collect preliminary results that we shall use in the proof of the square integrability. The sixth section states the main result of the paper. In the seventh section, we prove surjectivity for triples $\left(\pi_{c u s p}, \varphi, \Delta_{\epsilon}\right)$ in the case that $\varphi, \epsilon$ are alternated, i.e. satisfy the above condition (1) with respect to its partial cuspidal support (this is clearly a property of $\Delta_{\epsilon}$; it does not depend on a choice of $\epsilon$ ). We call this the alternated case. The other case is called the mixed case. The proof in the mixed case proceeds by induction.

Suppose that $\pi_{\text {cusp }}$, Jord (i.e. $\varphi$ ) and $\epsilon$ are fixed, and that

$$
\operatorname{Jord}\left(\pi_{\text {cusp }}\right)=\operatorname{Jord}\left(\varphi_{\varphi, \epsilon, \text { cusp }}\right) .
$$


Let $\left(\rho, a_{-}\right),(\rho, a) \in J_{o r d}, a_{-}<a$, be such that $\epsilon\left(\rho, a_{-}\right)=\epsilon(\rho, a)$ and $(\rho, b) \notin J o r d$ for $a_{-}<b<a$. Define $\varphi_{1}, \epsilon_{1}$ (and $G_{1}$ ) in the same way as we did in the definition of $\left(\varphi_{\varphi, \epsilon, c u s p}, \epsilon_{\varphi, \epsilon, c u s p}\right)$. By the inductive assumption, we know that there exists an irreducible square integrable representation $\pi_{1}$ of $G_{1}$ corresponding to $\left(\pi_{\text {cusp }}, \varphi_{1}, \Delta_{\epsilon_{1}}\right)$. Denote by $\delta\left(\left[\nu^{-\left(a_{-}-1\right) / 2} \rho, \nu^{(a-1) / 2} \rho\right]\right)$ the irreducible essentially square integrable representation of $G L\left(d_{\rho}\left(a+a_{-}\right) / 2, F\right)$, where $\rho$ is a representation of $G L\left(d_{\rho}, F\right)$, which is a unique irreducible subrepresentation of the induced representation

$$
\nu^{(a-1) / 2} \rho \times \nu^{(a-1) / 2-1} \times \cdots \times \nu^{-\left(a_{-}-1\right) / 2} \rho .
$$

It is proved in [M2] (and recalled here) that the induced representation

$$
\delta\left(\left[\nu^{-\left(a_{-}-1\right) / 2} \rho, \nu^{(a-1) / 2} \rho\right]\right) \rtimes \pi_{1}
$$

has exactly two irreducible subrepresentations, and that these subrepresentations are not isomorphic. For the proof of the surjectivity of the mapping

$$
\pi \mapsto\left(\pi_{\text {cusp }}, \operatorname{Jord}(\pi), \Delta_{\pi}\right),
$$

it is enough to prove that these two subrepresentations are square integrable. This is enough for the following reason. If we know that these subrepresentations are square integrable, then their parameters must be among those computed in [M2]. They are extensions of $\varphi_{1}, \Delta_{\epsilon_{1}}$. Since there exist exactly two parameters for $G$ which extend $\varphi_{1}, \Delta_{\epsilon_{1}}$ in this case, we get that they correspond to these irreducible square integrable subrepresentations, and one of them must correspond to $\varphi, \Delta_{\epsilon}$.

The square integrability of these irreducible subrepresentations is the most difficult part of the paper. We first solve the case where $\pi_{1}$ is cuspidal in the ninth section (earlier, in the eighth section we prove a very useful lemma about tempered representations with the same infinitesimal characters). In section 10, we prove the square integrability in the case when all the Jacquet modules of $\pi_{1}$ have good properties relative to $a$ and $a_{-}$(this includes for example the case when $a$ is the greatest element such that $(\rho, a) \in J$ ord $)$. Section 11 completes the proof, arguing with an inductive argument with respect to $a$.

In section 12, we discuss very briefly the basic assumption. Section 13 describes the irreducible tempered representations. Tempered representations can also be classified with triples as above. We have only to suppress the condition that the Jord's are multiplicity free. We have not developed this point of view here, which is by our basic assumption a direct consequence of Harish-Chandra's result on the intertwining algebras. Section 14 proves two exercises which we have mentioned earlier in this introduction, and gives simple examples of admissible triples. At the end, sections 15 and 16 explain modifications and comments which are necessary for the classifications obtained in previous sections, and proofs, to hold for unitary and even-orthogonal groups.

\section{Notation}

In this section, first we shall recall some notation for general linear groups (more details can be found in $[\mathrm{Z}]$ ).

We fix a local non-archimedean field $F$. We do not assume any restriction on the characteristic, but the reader has to be aware that our basic assumption (BA) has only been verified in [M5]) under the assumption of some Arthur's conjectures, which need the hypothesis that the characteristic of the field is 0 . 
By $F^{\prime}$ we shall denote in this paper either $F$ or a separable quadratic extension of $F$. This will depend on the following: if we are working with symplectic or orthogonal groups, then $F^{\prime}$ will denote $F$, and if we are working with unitary groups, then $F^{\prime}$ will denote the separable quadratic extension which enters the definition of the unitary groups.

If $F^{\prime}$ is a separable quadratic extension, we shall denote by $\theta$ the non-trivial element of the Galois group $\operatorname{Gal}\left(F^{\prime} / F\right)$ of $F^{\prime}$ over $F$. Otherwise (i.e. if $F^{\prime}=F$ ), $\theta$ will denote the identity mapping on $F$.

The modulus character of $F^{\prime}$ will be denoted by ||$_{F^{\prime}}$.

By a representation of a reductive group $G$ over $F$, we shall always mean in this paper a smooth representation. We denote by $\mathfrak{R}(G)$ the Grothendieck group of the category of all representations of $G$ of finite length. There is a natural ordering $\leq$ on $\mathfrak{R}(G)$. These orderings also determine natural ordering on a direct sum of Grothendieck groups of categories, for different reductive groups. For a finite length representation $\pi$ of $G$, we shall denote its semi-simplification by s.s. $(\tau)$, and consider it as an element of $\mathfrak{R}(G)$. For two finite length representations $\pi_{1}, \pi_{2}$ of $G$, we will shorten s.s. $\left(\pi_{1}\right) \leq$ s.s. $\left(\pi_{2}\right)$ to $\pi_{1} \leq \pi_{2}$.

For two representations $\pi_{1}, \pi_{2}$, of general linear groups over $F^{\prime}$, Bernstein and Zelevinsky defined a parabolically induced representation $\pi_{1} \times \pi_{2}$ in a natural way (see [Z]). Denote $R=\bigoplus_{n \geq 0} \mathfrak{R}\left(G L\left(n, F^{\prime}\right)\right)$. Then $\times$ factors in a natural way to $\times: R \times R \rightarrow R$. The multiplication factors through $R \otimes R$ in a unique way by a map, which will be denoted by $m: R \times R \rightarrow R$.

Recall that Bernstein and Zelevinsky also defined a comultiplication $m^{*}: R \rightarrow$ $R \otimes R$ using Jacquet modules. We shall briefly recall the definition of $m^{*}$ (for more details see $[\underline{\mathrm{Z}})$. For integers $0 \leq k \leq n$, there exists a unique standard parabolic subgroup $P_{(k, n-k)}=M_{(k, n-k)} N_{(k, n-k)}$ of $G L\left(n, F^{\prime}\right)$ whose Levi factor $M_{(k, n-k)}$ is naturally isomorphic to $G L\left(k, F^{\prime}\right) \times G L\left(n-k, F^{\prime}\right)$ (the standard parabolic subgroup is that one which contains the Borel subgroup consisting of an upper triangular in $\left.G L\left(n, F^{\prime}\right)\right)$. The normalized Jacquet module of a finite length representation $\pi$ of $G L\left(n, F^{\prime}\right)$ with respect to $P_{(k, n-k)}$ will be denoted by $r_{(k, n-k)}(\pi)$. Now one can view in a natural way

$$
\text { s.s. }\left(r_{(k, n-k)}(\pi)\right) \in \mathfrak{R}\left(G L\left(k, F^{\prime}\right)\right) \otimes \mathfrak{R}\left(G L\left(n-k, F^{\prime}\right)\right) \subseteq R \otimes R .
$$

Define

$$
m^{*}(\pi)=\sum_{k=0}^{n} \text { s.s. }\left(r_{(k, n-k)}(\pi)\right) \in R \otimes R .
$$

Extend $m^{*}$ additively to a mapping $m^{*}: R \rightarrow R \otimes R$. With these two operations, $m$ and $m^{*}, R$ is a Hopf algebra. In particular, $m^{*}$ is multiplicative (we shall use this property quite often, very often even without mentioning it).

The character $|\operatorname{det}|_{F^{\prime}}$ of $G L\left(n, F^{\prime}\right)$ will be denoted by $\nu$. For an irreducible essentially square integrable representation $\delta$ of $G L\left(n, F^{\prime}\right)$, there exist a unique $e(\delta) \in \mathbb{R}$ and an irreducible unitarizable square integrable representation $\delta^{u}$ such that

$$
\delta=\nu^{e(\delta)} \delta^{u} .
$$

Let $\rho$ be an irreducible cuspidal representation of a general linear group (over $\left.F^{\prime}\right)$. For $n \in \mathbb{Z}_{+}$, denote

$$
\left[\rho, \nu^{n} \rho\right]=\left\{\rho, \nu \rho, \ldots, \nu^{n} \rho\right\}
$$


$\left(\mathbb{Z}_{+}\right.$denotes the set of all non-negative integers). We shall call $\Delta=\left[\rho, \nu^{n} \rho\right]$ a segment (in irreducible cuspidal representations of general linear groups). The representation $\rho \times \nu \rho \times \cdots \times \nu^{n} \rho$ has a unique irreducible quotient (resp. irreducible subrepresentation), which we denote by $\delta(\Delta)($ resp. $\mathfrak{s}(\Delta))$. The representation $\delta(\Delta)$ is essentially square integrable. For $n<0$, we shall assume $\left[\rho, \nu^{n} \rho\right]$ to be $\emptyset$. We take $\delta(\emptyset)=1$ (identity in $R$, which is the trivial representation of $G L\left(0, F^{\prime}\right)$ ).

We shall recall now some notation for classical $p$-adic groups. More details regarding this notation can be found in $\mathrm{MViW}$ and $\mathrm{T5}$. We shall consider the following series $S_{n}$ of classical groups over $F$ (and we shall fix one of these series of classical groups).

Symplectic groups $S p(2 n, F)$ form a series of groups where $n$ denotes the split semi-simple rank of the group. The group $S p(2 n, F)$ will be denoted by $S_{n}$. We have here the notion of the Witt tower (we shall denote by $V_{n}$ the symplectic space of dimension $2 n$ in this tower; we shall denote $V_{0}$ also by $Y_{0}$ in the case of symplectic groups).

In the case of odd-orthogonal groups, we also have the Witt tower: this means that we fix an anisotropic orthogonal vector space $Y_{0}$ over $F$ of odd dimension (1 or 3), and we look at the Witt tower based on $Y_{0}$. For each $n$ such that $2 n+1 \geq$ $\operatorname{dim} Y_{0}$, there is exactly one space $V_{n}$ in the Witt tower whose dimension is $2 n+1$. We denote by $S_{n}$ the special orthogonal group of this space (recall that in this case the orthogonal group of $V_{n}$ is a product of its center and $S_{n}$ ).

In the case of even-orthogonal groups, we fix an anisotropic orthogonal space $Y_{0}$ over $F$ of even dimension, and consider the Witt tower based on $Y_{0}$. If $2 n \geq$ $\operatorname{dim}_{F}\left(Y_{0}\right)$, then there is exactly one space $V_{n}$ in the Witt tower of dimension $2 n$. The orthogonal group of $V_{n}$ will be denoted by $S_{n}$.

In the case of symplectic or orthogonal series $S_{n}$ of groups, $F^{\prime}$ will denote $F$.

In the case of unitary groups, $F^{\prime}$ will denote a separable quadratic extension of $F$. We shall fix an anisotropic unitary space $Y_{0}$ over $F^{\prime}$, and consider the Witt tower of unitary spaces $V_{n}$ based on $Y_{0}$.

Suppose that $\operatorname{dim}_{F^{\prime}}\left(Y_{0}\right)$ is odd (i.e. 1). Then for each $2 n+1 \geq \operatorname{dim}_{F^{\prime}}\left(Y_{0}\right)$, there exists a unique space $V_{n}$ in the Witt tower of dimension $2 n+1$. The unitary group of this space will be denoted by $S_{n}$.

If $\operatorname{dim}_{F^{\prime}}\left(Y_{0}\right)$ is even, then for each $2 n \geq \operatorname{dim}_{F^{\prime}}\left(Y_{0}\right)$ one takes a unique space $V_{n}$ in the Witt tower of dimension $2 n$, and denotes its unitary group by $S_{n}$.

We fix a minimal parabolic subgroup in $S_{n}$. We shall consider in this paper only standard parabolic subgroups, i.e. the parabolic subgroups which contain the fixed minimal parabolic subgroup. For more information regarding convenient matrix realizations of groups $S_{n}$ and for a description of their standard parabolic subgroups for $S O(s n+1, F)$ and $S p(2 n, F)$, one can consult [T5].

Fix one of the series $S_{n}$ of groups that we have defined. Let $n^{\prime}$ be the Witt index of $V_{n}$ ( $n^{\prime}$ is $n-1 / 2 \operatorname{dim}_{F^{\prime}}\left(Y_{0}\right)$ if $V_{n}$ is a symplectic or even-orthogonal or evenunitary group, otherwise $n^{\prime}$ is $\left.n-1 / 2\left(\operatorname{dim}_{F^{\prime}}\left(Y_{0}\right)-1\right)\right)$. For each $0 \leq k \leq n^{\prime}$, there exists a standard parabolic subgroup $P_{(k)}=M_{(k)} N_{(k)}$ of $S_{n}$, whose Levi subgroup $M_{(k)}$ is naturally isomorphic to $G L\left(k, F^{\prime}\right) \times S_{n-k}$ (see [T5] and [B] for the series of symplectic and split orthogonal groups; the isomorphism in the case of other series of groups is defined in an analogous way). This parabolic subgroup is the stabilizer of an isotropic space of dimension $k$. For representations $\pi$ and $\sigma$ of $G L\left(k, F^{\prime}\right)$ and $S_{n-k}$ respectively, the representation parabolically induced by $\pi \otimes \sigma$ is denoted by 
A simple but very useful property of $\rtimes$ is that for two representations $\pi_{1}$ and $\pi_{2}$ of general linear groups (over $F^{\prime}$ ) we have

$$
\pi_{1} \rtimes\left(\pi_{2} \rtimes \sigma\right) \cong\left(\pi_{1} \times \pi_{2}\right) \rtimes \sigma
$$

(see [T5]).

For $\pi$ as above, denote by $\check{\pi}$

$$
g \mapsto \tilde{\pi}(\theta(g)),
$$

where $\tilde{\pi}$ denotes the contragredient representation of $\pi$. If $\pi$ and $\sigma$ are representations of finite length, then

$$
\text { S.s. }(\pi \rtimes \sigma)=\text { s.s. }(\check{\pi} \rtimes \sigma) \text {. }
$$

In particular, if $\pi \rtimes \sigma$ is irreducible, then $\pi \rtimes \sigma \cong \check{\pi} \rtimes \sigma$.

We shall say that a representation $\pi$ of a general linear group over $F^{\prime}$ is $F^{\prime} / F$ selfdual if

$$
\pi \cong \check{\pi}
$$

If $F^{\prime}=F$, then we shall also simply say that $\pi$ is selfdual.

For a representation $\tau$ of $S_{n}$, we denote the normalized Jacquet module of $\tau$ with respect to $P_{(k)}$ by $s_{(k)}(\tau)$. All the Jacquet modules that we shall consider in this paper will be normalized Jacquet modules with respect to standard parabolic subgroups (and obvious Levi decompositions).

Denote $R(S)=\bigoplus \mathfrak{R}\left(S_{n}\right)$, where the sum runs over all the groups from the series $S_{n}$ of the classical groups that we have fixed (in other words, the sum runs over all integers $n \geq 1 / 2\left(\operatorname{dim}_{F^{\prime}}\left(Y_{0}\right)-1\right)$ if we consider odd-orthogonal or odd-unitary groups, and over all $n \geq 1 / 2 \operatorname{dim}_{F^{\prime}}\left(Y_{0}\right)$ otherwise). Then $\rtimes$ defines in a natural way a mapping $\rtimes: R \times R(S) \rightarrow R(S)$. Let

$$
\mu^{*}(\tau)=\sum_{k=0}^{n^{\prime}} \text { S.S. }\left(s_{(k)}(\tau)\right),
$$

where $\tau$ is an irreducible representation of $S_{n}\left(n^{\prime}\right.$ is the Witt index of $\left.V_{n}\right)$. Extend $\mu^{*}$ additively to $\mu^{*}: R(S) \rightarrow R \otimes R(S)$.

Let $\quad M^{*}=(m \otimes 1) \circ\left({ }^{`} \otimes m^{*}\right) \circ \kappa \circ m^{*}: R \rightarrow R \otimes R$, where ${ }^{`}: R \rightarrow R$ is a homomorphism defined by $\pi \mapsto \check{\pi}$ on irreducible representations, and $\kappa: R \times R \rightarrow$ $R \times R$ maps $\sum x_{i} \otimes y_{i}$ to $\sum y_{i} \otimes x_{i}$. Note that $M^{*}(\pi)$ depends also on the series $S_{n}$ of the groups that we have fixed.

For a finite length representation $\pi$ of $G L\left(k, F^{\prime}\right)$, the component of $M^{*}(\pi)$ which is in $\mathfrak{R}\left(G L\left(k, F^{\prime}\right)\right) \otimes \mathfrak{R}\left(G L\left(0, F^{\prime}\right)\right)$ will be denoted by

$$
M_{G L}^{*}(\pi) \otimes 1 \text {. }
$$

For a finite length representation $\tau$ of $S_{q}, \mu^{*}(\tau)$ will denote $\mu^{*}$ (s.s. $\left.(\tau)\right)$. A similar convention will be used for $M^{*}$ and $M_{G L}^{*}$.

Let $\pi$ be a representation of $G L\left(k, F^{\prime}\right)$ of finite length and let $\sigma$ be an irreducible cuspidal representation of $S_{n}$. Suppose that $\tau$ is a subquotient of $\pi \rtimes \sigma$. Then we shall denote $s_{(k)}(\tau)$ also by

$$
s_{G L}(\tau) .
$$

In the sequel, until the end of section 14, we shall assume that the series of groups $S_{n}$ consists of symplectic or odd-orthogonal groups. The main reason for this restriction is to simplify the exposition in these sections. The proofs for these 
groups apply also to even-orthogonal and unitary groups (the notation that we use is prepared also for those cases). In sections 15 and 16 we shall describe differences and comment on the case of unitary and even-orthogonal groups respectively. Recall that for symplectic and orthogonal groups we have $F^{\prime}=F$ and $\check{\pi}=\tilde{\pi}$. Nevertheless, we shall also use in sections $2-14$ the notations $F^{\prime}$ and $\check{\pi}$ (instead of $F$ and $\pi$, which we could also use). The reason for this is that with such a choice of notation, sections 2-14 will also apply to unitary groups, after a few comments, without any significant changes.

For $\pi \in R$ and $\sigma \in R(S)$ we have

$$
\mu^{*}(\pi \rtimes \sigma)=M^{*}(\pi) \rtimes \mu^{*}(\sigma) .
$$

For split groups $S_{n}$, this follows from Theorems 5.4 and 6.5 of [T5]. For a non-split odd-orthogonal series of groups $S_{n}$, we have the same root system of type $B$ as in the split case, and the same Weyl group. Now the above formula for non-split oddorthogonal groups follows in the same way as in section 6 of [T5]. More precisely, the calculations in section 4 of [T5] are independent of the groups (but depend on the root systems), and they imply (an analogue of) Lemma 5.1 for non-split oddorthogonal groups. Now formula (1-1) follows from this lemma in completely the same way as T5, Theorem 5.2, follows from Lemma 5.1, by a formal computation* *

Let $\pi$ be a finite length representation of a general linear group and let $\tau$ be a similar representation of $S_{n}$. Then (1-1) implies

$$
\text { s.s. }\left(s_{G L}(\pi \rtimes \tau)\right)=M_{G L}^{*}(\pi) \times \text { s.s. }\left(s_{G L}(\tau)\right)
$$

( $\times$ in the above formula denotes multiplication in $R$ of $M^{*}(\pi)$ with the factors on the left-hand side of $\otimes$ in s.s. $\left.\left(s_{G L}(\tau)\right)\right)$.

In this paper, we shall use formulas for $M^{*}(\delta(\Delta))$ and $M_{G L}^{*}(\delta(\Delta))$ several times. We write these formulas here for the segments that we shall consider most often. Let $a_{-}, a \in \mathbb{N}\left(\mathbb{N}\right.$ denotes the positive integers). Suppose $a-a_{-} \in 2 \mathbb{N}$. Let $\rho$ be an irreducible $F^{\prime} / F$-selfdual cuspidal representation of a general linear group. Then the formula for $m^{*}(\delta(\Delta))$ implies

$$
\begin{gathered}
M^{*}\left(\delta\left(\left[\nu^{-\left(a_{-}-1\right) / 2} \rho, \nu^{(a-1) / 2} \rho\right]\right)\right) \\
=\sum_{i=-\left(a_{-}-1\right) / 2-1}^{(a-1) / 2} \sum_{j=i}^{(a-1) / 2} \delta\left(\left[\nu^{-i} \rho, \nu^{(a--1) / 2} \rho\right]\right) \\
\times \delta\left(\left[\nu^{j+1} \rho, \nu^{(a-1) / 2} \rho\right]\right) \otimes \delta\left(\left[\nu^{i+1} \rho, \nu^{j} \rho\right]\right) .
\end{gathered}
$$

To get $M_{G L}^{*}$, we need to take $j=i$ in the above formula. Thus

$$
\begin{aligned}
M_{G L}^{*}\left(\delta\left(\left[\nu^{-\left(a_{-}-1\right) / 2} \rho, \nu^{(a-1) / 2} \rho\right]\right)\right) & \\
= & \sum_{i=-\left(a_{-}-1\right) / 2-1}^{(a-1) / 2} \delta\left(\left[\nu^{-i} \rho, \nu^{\left(a_{-}-1\right) / 2} \rho\right]\right) \times \delta\left(\left[\nu^{i+1} \rho, \nu^{(a-1) / 2} \rho\right]\right) .
\end{aligned}
$$

* The second author is thankful to T. Springer who informed him in 1997 about the paper "An application of Hopf-algebra techniques to representations of finite classical groups" by Marc A. A. van Leeuwen (Journal of Algebra 140 (1991), 210-246). In that paper, formula (1-1) is computed in the case of finite classical groups (Theorem 3.2.1). Note that the formula is simpler in the finite field case. The reason for this is the cocommutativity of the Hopf algebra attached to representations of finite general linear groups. 
Denote by $\gamma$ that part of the sum in (1-4) corresponding to indexes

$$
\begin{gathered}
-\left(a_{-}-1\right) / 2-1 \leq i \leq\left(a_{-}-1\right) / 2 \\
\left(\text { resp. }-\left(a_{-}-1\right) / 2-1 \leq i \leq\left(a_{-}-1\right) / 2-1\right) .
\end{gathered}
$$

Then $\gamma$ satisfies condition (3-9) (resp. (3-10)) of Lemma 3.5.

Finally, we have

$$
\begin{aligned}
M^{*}\left(\delta\left(\left[\nu^{-\left(a_{-}-1\right) / 2} \rho, \nu^{\left(a_{-}-1\right) / 2} \rho\right]\right)\right) & \\
=\sum_{i^{\prime}=-\left(a_{-}-1\right) / 2-1}^{\left(a_{-}-1\right) / 2} & \sum_{j^{\prime}=i^{\prime}}^{\left(a_{-}-1\right) / 2} \delta\left(\left[\nu^{-i^{\prime}} \rho, \nu^{\left(a_{-}-1\right) / 2} \rho\right]\right) \\
& \times \delta\left(\left[\nu^{j^{\prime}+1} \rho, \nu^{\left(a_{-}-1\right) / 2} \rho\right]\right) \otimes \delta\left(\left[\nu^{i^{\prime}+1} \rho, \nu^{j^{\prime}} \rho\right]\right) .
\end{aligned}
$$

Clearly, $M_{G L}^{*}\left(\delta\left(\left[\nu^{-\left(a_{-}-1\right) / 2} \rho, \nu^{\left(a_{-}-1\right) / 2} \rho\right]\right)\right)$ satisfies condition (3-9) of Lemma 3.5.

\section{INVARIANTS OF SQUARE INTEGRABLE REPRESENTATIONS OF CLASSICAL GROUPS}

In this section we shall recall the invariants attached in $\mathrm{M} 2$ to square integrable representations, and some of their properties.

Let $y \in \mathbb{N}$. If we are considering the series of groups $S p(2 n)($ resp. $S O(2 n+1)$ ), then $R_{y}$ will denote the representation of $G L(y, \mathbb{C})$ on $\wedge^{2} \mathbb{C}^{y}\left(\right.$ resp. $\left.\operatorname{Sym}^{2} \mathbb{C}^{y}\right)$.

To any irreducible square integrable representation $\pi$ of $S_{n}$, [M2 associates three objects:

$$
\operatorname{Jord}(\pi), \pi_{\text {cusp }} \text { and } \epsilon_{\pi} .
$$

By definition, $(\rho, a) \in \operatorname{Jord}(\pi)$ if and only if $\rho$ is an irreducible $F^{\prime} / F$-selfdual cuspidal representation of a general linear group $G L\left(d_{\rho}, F^{\prime}\right)$ (this defines $d_{\rho}$ ) and $a \in \mathbb{N}$ such that

$$
a \text { is even if } L\left(\rho, R_{d_{\rho}}, s\right) \text { has a pole at } s=0 \text {, and odd otherwise, }
$$

and

$$
\delta(\rho, a) \rtimes \pi_{0} \text { is irreducible, }
$$

where $\delta(\rho, a)$ denotes $\delta\left(\left[\nu^{-(a-1) / 2} \rho, \nu^{(a-1) / 2} \rho\right]\right)$.

For an irreducible $F^{\prime} / F$-selfdual cuspidal representation of a general linear group $\rho$, denote

$$
\operatorname{Jord}_{\rho}(\pi)=\{a ;(\rho, a) \in \operatorname{Jord}(\pi)\} .
$$

In the above definition, $L\left(\rho, R_{d_{\rho}}, s\right)$ is the $L$-function defined by Shahidi. We only need a definition and we do not use any property of the $L$-functions except in section 12. Therefore, we could also take $L\left(\rho, R_{d_{\rho}}, s\right)$ to be the $L$-function defined via the Langlands correspondence on the Galois side. Henniart has announced recently that both definitions give the same $L$-function. The basic problem in the definition of the Jordan block is to give a definition of the parity of $a$ 's for which $(\rho, a)$ lies in the Jordan block in a such a way that (BA) holds. In [M5] we have given another definition which depends on the cuspidal support, but which avoids any use of $L$-functions (Remark 2.3 of this paper gives one possible definition of the Jordan block without use of $L$-functions; further Remark 14.5 is also along these lines). 
Let $\pi_{c u s p}$ be an irreducible cuspidal representation of some $S_{q}$ and let $\rho$ be an irreducible $F^{\prime} / F$-selfdual cuspidal representation of a general linear group. In this paper we shall assume that the following basic assumption holds:

$$
\begin{aligned}
& \nu^{ \pm\left(a_{\rho}+1\right) / 2} \rho \rtimes \pi_{\text {cusp }} \text { reduces for } \\
& a_{\rho}=\left\{\begin{array}{l}
\max \operatorname{Jord}_{\rho}\left(\pi_{\text {cusp }}\right) \quad \text { if } \operatorname{Jord}_{\rho}\left(\pi_{\text {cusp }}\right) \neq \emptyset, \\
0 \quad \text { if } L\left(\rho, R_{d_{\rho}}, s\right) \text { has a pole at } s=0 \text { and } \operatorname{Jord}_{\rho}\left(\pi_{\text {cusp }}\right)=\emptyset, \\
-1 \quad \text { otherwise; }
\end{array}\right. \\
& \text { moreover, there are no other reducibility points in } \mathbb{R} \text {. }
\end{aligned}
$$

Note that a part of the assumption (BA) is that $\operatorname{Jord}_{\rho}\left(\pi_{\text {cusp }}\right)$ are finite sets. An additional comment regarding this conjecture can be found in section 12.

The partial cuspidal support of $\pi, \pi_{c u s p}$, is defined to be the cuspidal irreducible representation of $S_{n^{\prime}}$, with $n^{\prime} \leq n$, such that there exist an irreducible representation $\sigma$ of $G L\left(n-n^{\prime}, F^{\prime}\right)$ and an embedding

$$
\pi \hookrightarrow \sigma \times \pi_{\text {cusp }} .
$$

Note that the notion of partial cuspidal support is well defined in this way for any irreducible representation of $S_{n}$.

Let $\pi$ be an irreducible square integrable representation of $S_{n}$ and let $\pi_{\text {cusp }}$ be the partial support of $\pi$, where $\pi_{\text {cusp }}$ is a cuspidal representation of some $S_{n^{\prime}}\left(n^{\prime} \leq n\right)$. We know that the following inequality holds:

$$
\sum_{(\rho, a) \in \operatorname{Jord}\left(\pi_{\text {cusp }}\right)} a d_{\rho} \leq \begin{cases}2 n^{\prime} & \text { if } S_{n^{\prime}}=S O\left(2 n^{\prime}+1\right), \\ 2 n^{\prime}+1 & \text { if } S_{n^{\prime}}=S p(2 n) .\end{cases}
$$

This inequality has been proved recently by elementary methods without assuming any hypothesis (see Corollary 4.3 of [M5]). Let us note that it is expected that the equality holds in (A). To prove the equality, it remains to prove the other inequality. A difficult problem would be to prove this other inequality $\geq$ in (A). Using (BA) it is proved in $\mathrm{M} 2$ that

$$
\sum_{(\rho, a) \in \operatorname{Jord}(\pi)} a d_{\rho}-\sum_{\left(\rho^{\prime}, a^{\prime}\right) \in \operatorname{Jor} d\left(\pi_{c u s p}\right)} a^{\prime} d_{\rho^{\prime}}=2\left(n-n^{\prime}\right)
$$

(see 4.1 and 4.2 there). Now (A) and (2-1) imply that $\operatorname{Jord}(\pi)$ is finite. This is not a very deep result. In fact, we hope that for an irreducible cuspidal representation $\pi$ and an odd-orthogonal group $G, \operatorname{Jord}(\pi)$ determines $\pi$ (this is proved in some very special cases in [M5]). The first information that we would need in that direction is that $\operatorname{Jord}(\pi)$ is big enough!

At the end, M2 associates to $\pi$ a partially defined function $\epsilon_{\pi}$ from $\operatorname{Jord}\left(\pi_{0}\right)$ into $\{ \pm 1\}$ (we shall explain below precisely what we mean by partially defined function). The definition of $\epsilon_{\pi}$ is in terms of Jacquet modules of $\pi$, except in one case, in which normalized intertwining operators are used. Let $(\rho, a) \in \operatorname{Jord}(\pi)$. Assume that there exists $a^{\prime}$ such that $\left(\rho, a^{\prime}\right) \in \operatorname{Jord}(\pi)$ and $a^{\prime}<a$. Let $a_{-}$be the biggest possible such $a^{\prime}$. In that case, we define:

$$
\begin{array}{ll}
\epsilon_{\pi}(\rho, a) \epsilon_{\pi}\left(\rho, a_{-}\right)=1 \quad \Leftrightarrow \quad \text { there exists a representation } \pi^{\prime} \text { of } S_{n-\left(a-a_{-}\right) / 2} \\
\text { such that } \pi \hookrightarrow \delta\left(\left[\nu^{\left(a_{-}+1\right) / 2} \rho, \nu^{(a-1) / 2} \rho\right]\right) \times \pi^{\prime} .
\end{array}
$$


This is the most important property of $\epsilon_{\pi}$. With such a definition, we can formally define $\epsilon_{\pi}(\rho, a) \epsilon_{\pi}\left(\rho, a^{\prime}\right)^{-1} \in\{ \pm 1\}$ for all $\rho, a, a^{\prime}$ such that $(\rho, a),\left(\rho, a^{\prime}\right) \in \operatorname{Jord}(\pi)$. Of course if $a=a^{\prime}$, then the number is 1 . If $(\rho, a) \in \operatorname{Jord}(\pi)$ and $a$ is even, then we put formally $\epsilon_{\pi}(\rho, 0)=1$ and allow $a^{\prime}=0$ in the above construction. In this way, $\epsilon_{\pi}(\rho, a)$ is defined for all $a$ even. There is one case of odd $a$ where we can and do define $\epsilon_{\pi}(\rho, a)$ : it is the case where $\rho$ is such that there exists $a$ odd with $(\rho, a) \in \operatorname{Jord}(\pi)$ but there does not exist $b$ such that $(\rho, b) \in \operatorname{Jord}\left(\pi_{\text {cusp }}\right)\left(\pi_{\text {cusp }}\right.$ is the partial cuspidal support of $\pi$ ). In this case the definition is not canonical. Now (BA) in this case implies that the induced representation $\rho \times \pi_{\text {cusp }}$ is reducible. It reduces in the sum of two inequivalent irreducible subrepresentations. We choose one of those irreducible subrepresentations. After this choice, we can define $\epsilon_{\pi}(\rho, a)$ for any $a$ such that $(\rho, a) \in \operatorname{Jord}(\pi)$. This is done in [M2] (beginning of section 6). In this way $\epsilon_{\pi}(\rho, a) \epsilon_{\pi}\left(\rho, a^{\prime}\right)^{-1}$ is defined for all $\left(\rho, a, a^{\prime}\right)$ such that $(\rho, a),\left(\rho, a^{\prime}\right) \in$ $\operatorname{Jord}(\pi)$. Further $\epsilon_{\pi}(\rho, a)$ is defined if $(\rho, a) \in \operatorname{Jord}(\pi)$ and $a$ is even, or $a$ odd but there does not exists $b$ such that $(\rho, b) \in \operatorname{Jord}\left(\pi_{\text {cusp }}\right)$.

In [M2] it is proved that $\operatorname{Jord}\left(\pi_{0}\right), \pi_{c u s p}, \epsilon_{\pi_{0}}$ form an admissible triple. A general definition of an admissible triple will be recalled below. The fundamental result of [M2] (see section 7 of [M2]) is that the mapping

$$
\pi \mapsto\left(\operatorname{Jord}(\pi), \pi_{\text {cusp }}, \epsilon_{\pi}\right)
$$

is an injective mapping from the set of all equivalence classes of irreducible square integrable representations of groups $S_{n}$ into the set of all admissible triples (assuming that (BA) holds). Before we give the definition of an admissible triple, we shall give two remarks which are related to the admissibility condition.

The first one is a result which we shall use later, which tells us that, for an irreducible cuspidal representation $\pi_{\text {cusp }}$ of $S_{n}$, the following holds:

$$
(\rho, a) \in \operatorname{Jord}\left(\pi_{\text {cusp }}\right) \text { and } a>2 \quad \Rightarrow \quad(\rho, a-2) \in \operatorname{Jord}\left(\pi_{\text {cusp }}\right) .
$$

In M1 this assertion and (BA) are obtained simultaneously as consequences of some Arthur's conjectures. One can check easily that (BA) implies (2-3) (one can find the proof of this implication in section 12). In fact, (2-3) follows from the fact that there is only one reducibility point in $\{x \in \mathbb{R} ; x \geq 0\}$ in the case of the parabolic induction from a maximal parabolic subgroup by an irreducible cuspidal representation.

For an irreducible square integrable representation $\pi$ of $S_{n}$ define the multiset

$$
\operatorname{Supp}(\operatorname{Jord}(\pi))=\sum_{(\rho, a) \in \operatorname{Jord}(\pi)}\left[\nu^{-(a-1) / 2} \rho, \nu^{(a-1) / 2} \rho\right]
$$

(multiset means that elements are counted with multiplicity; see [Z] ). It is evident how to reconstruct $\operatorname{Jord}(\pi)$ from the multiset $\operatorname{Supp}(\operatorname{Jord}(\pi))$. Now

$$
\operatorname{Supp}\left(\operatorname{Jord}\left(\pi_{\text {cusp }}\right)\right) \subseteq \operatorname{Supp}(\operatorname{Jord}(\pi)) .
$$

The above inclusion follows from the admissibility of the triple $\left(\operatorname{Jord}(\pi), \pi_{\text {cusp }}, \epsilon_{\pi}\right)$.

Now we shall define the notion of an admissible triple.

Fix an irreducible cuspidal representation $\pi_{\text {cusp }}$ of some $S_{q}$. Let Jord be a finite set of pairs $(\rho, a)$, where $\rho$ are $F^{\prime} / F$-selfdual irreducible cuspidal representations of general linear groups and $a \in \mathbb{N}$ is such that condition (J-1) holds for each of these pairs. We shall say that Jord has degree $n$ if (2-1) holds (the sum in (2-1) runs over Jord instead of $\operatorname{Jor} d(\pi))$. We define the multiset $\operatorname{Supp}(J$ ord $)$ in the same way as we did for $\operatorname{Jord}(\pi)$. For an $F^{\prime} / F$-selfdual irreducible cuspidal representation $\rho$ 
of a general linear group, one defines $\operatorname{Jord}_{\rho}=\{a ;(\rho, a) \in \operatorname{Jord}\}$. For $a \in \operatorname{Jord}_{\rho}$ we denote

$$
a_{-}=\max \left\{b \in \operatorname{Jord}_{\rho} ; b<a\right\},
$$

if $\left\{b \in \operatorname{Jord}_{\rho} ; b<a\right\} \neq \emptyset$ (and then we say that $a_{-}$exists, or that it is defined). Otherwise, we shall say that $a$ does not have $a_{-}$, or that $a$ is minimal in $\operatorname{Jord}_{\rho}$.

Let $\epsilon$ be a partially defined function from a subset of $\operatorname{Jord} \cup($ Jord $\times$ Jord $)$ into $\{ \pm 1\}$ in the following sense. For $(\rho, a) \in \operatorname{Jord}, \epsilon(\rho, a)$ is not defined if and only if $a$ is odd and $\left(\rho, a^{\prime}\right) \in \operatorname{Jord}\left(\pi_{\text {cusp }}\right)$ for some $a^{\prime} \in \mathbb{N}$. Further $\epsilon$ is defined on a pair $(\rho, a),\left(\rho^{\prime}, a^{\prime}\right) \in$ Jord if and only if $\rho=\rho^{\prime}$ and $a \neq a^{\prime}$.

We shall require that $\epsilon$ satisfies the following properties. Let $(\rho, a),\left(\rho, a^{\prime}\right) \in$ Jord, $a \neq a^{\prime}$. If $\epsilon(\rho, a)$ is defined, then $\epsilon$ on this pair has the value

$$
\epsilon(\rho, a) \epsilon\left(\rho, a^{\prime}\right)^{-1} \text {. }
$$

If $\epsilon(\rho, a)$ is not defined, then we shall also formally denote the value of $\epsilon$ on this pair by $\epsilon(\rho, a) \epsilon\left(\rho, a^{\prime}\right)^{-1}$ (although $\epsilon(\rho, a)$ and $\epsilon\left(\rho, a^{\prime}\right)$ are not defined). In this case (when $\epsilon(\rho, a)$ is not defined), for different $a, a^{\prime}, a^{\prime \prime} \in \operatorname{Jord}_{\rho}$ we require that

$$
\epsilon(\rho, a) \epsilon\left(\rho, a^{\prime \prime}\right)^{-1}=\left(\epsilon(\rho, a) \epsilon\left(\rho, a^{\prime}\right)^{-1}\right)\left(\epsilon\left(\rho, a^{\prime}\right) \epsilon\left(\rho, a^{\prime \prime}\right)^{-1}\right)
$$

(recall that the notation in the above formula is formal). In this case, we also require for different $a, a^{\prime} \in \operatorname{Jord}_{\rho}$ :

$$
\epsilon(\rho, a) \epsilon\left(\rho, a^{\prime}\right)^{-1}=\epsilon\left(\rho, a^{\prime}\right) \epsilon(\rho, a)^{-1} .
$$

In the case that $\epsilon(\rho, a) \epsilon\left(\rho, a^{\prime}\right)^{-1} \neq 1$ (resp. $\epsilon(\rho, a) \epsilon\left(\rho, a^{\prime}\right)^{-1}=1$ ), we shall write formally

$$
\epsilon(\rho, a) \neq \epsilon\left(\rho, a^{\prime}\right) \quad\left(\text { resp. } \epsilon(\rho, a)=\epsilon\left(\rho, a^{\prime}\right)\right),
$$

even if $\epsilon(\rho, a)$ and $\epsilon\left(\rho, a^{\prime}\right)$ are not defined.

Suppose that we have the triple Jord, $\pi_{c u s p}, \epsilon$ as above. Let $(\rho, a) \in$ Jord be such that $a_{-} \in \operatorname{Jord}_{\rho}$ is defined and

$$
\epsilon(\rho, a)=\epsilon\left(\rho, a_{-}\right) .
$$

Denote $J_{o r d}=\operatorname{Jord} \backslash\left\{a, a_{-}\right\}$. Let $\epsilon^{\prime}$ be the partially defined function on Jord which we get by restricting $\epsilon$ to $J o r d^{\prime}$. Then $\epsilon^{\prime}$ again satisfies the above requirements with respect to $J o r d^{\prime}$ and $\pi_{c u s p}$. If we have two triples $J_{o r d}, \pi_{c u s p}, \epsilon$ and $J \operatorname{Jord}^{\prime}, \pi_{\text {cusp }}, \epsilon^{\prime}$ as above, then we shall say that $J_{o r d}, \pi_{c u s p}, \epsilon^{\prime}$ is subordinated to Jord, $\pi_{\text {cusp }}, \epsilon$.

Let $J$ ord $, \pi_{\text {cusp }}, \epsilon$ be a triple as above. For $(\rho, a) \in$ Jord denote

$$
\operatorname{Jord}_{\rho}^{\prime}\left(\pi_{\text {cusp }}\right)= \begin{cases}\operatorname{Jord}_{\rho}\left(\pi_{\text {cusp }}\right) \cup\{0\} & \text { if } a \text { is even and } \epsilon\left(\rho, \min \operatorname{Jord}_{\rho}\right)=1, \\ \operatorname{Jord}_{\rho}\left(\pi_{\text {cusp }}\right) & \text { otherwise. }\end{cases}
$$

The two notations $J_{o r d}$ and $J_{o r d}\left(\pi_{\text {cusp }}\right)$ will not be used simultaneously, to avoid confusion.

Now we shall define the admissible triple. Let $J o r d, \pi_{c u s p}, \epsilon$ be a triple as above. First we have the following definition.

We shall say that Jord, $\pi_{c u s p}, \epsilon$ is an admissible triple of alternated type if for each $(\rho, a) \in$ Jord the following two conditions hold:

$$
\begin{aligned}
& \epsilon(\rho, a) \neq \epsilon\left(\rho, a_{-}\right) \quad \text { if } a_{-} \text {is defined, and } \\
& \text { card } \operatorname{Jord}_{\rho}^{\prime}\left(\pi_{\text {cusp }}\right)=\text { card } \operatorname{Jord}_{\rho} .
\end{aligned}
$$


We shall say that a triple $J o r d, \pi_{\text {cusp }}, \epsilon$ is admissible, if there exists a sequence of triples $\operatorname{Jord}_{i}, \pi_{\text {cusp }}, \epsilon_{i}, 1 \leq i \leq k$, such that

$\left(J o r d, \pi_{c u s p}, \epsilon\right)=\left(\operatorname{Jord}_{1}, \pi_{\text {cusp }}, \epsilon_{1}\right)$,

$\operatorname{Jord}_{i+1}, \pi_{\text {cusp }}, \epsilon_{i+1}$ is subordinated to $J_{o r d}, \pi_{c u s p}, \epsilon_{i}$ for $1 \leq i \leq k-1$, and

$\operatorname{Jord}_{k}, \pi_{\text {cusp }}, \epsilon_{k}$ is an admissible triple of alternated type.

An admissible triple which is not of alternated type will be called an admissible triple of mixed type.

Suppose that $J o r d, \pi_{c u s p}, \epsilon$ is an admissible triple of alternated type. Then (2-6) implies that for each $(\rho, a) \in$ Jord there exists a unique monotone bijection

$$
\phi_{\rho}: \operatorname{Jord}_{\rho} \rightarrow \operatorname{Jord}_{\rho}^{\prime}\left(\pi_{\text {cusp }}\right) \text {. }
$$

We shall prove that to each admissible triple $J o r d, \pi_{c u s p}, \epsilon$ of degree $n$ there is attached an irreducible square integrable representation $\pi$ of $S_{n}$ with that invariant, i.e. that $\left(\operatorname{Jord}(\pi), \pi_{\text {cusp }}, \epsilon_{\pi}\right)=\left(\operatorname{Jord}, \pi_{\text {cusp }}, \epsilon\right)$ (where $\pi_{\text {cusp }}$ is the partial cuspidal support of $\pi)$.

To illustrate the notion of $\operatorname{Jord}(\pi)$, we shall now give a proposition which is the basic method for computing $\operatorname{Jord}(\pi)$ from $\operatorname{Jord}\left(\pi_{\text {cusp }}\right)$. This result is already contained in [M2]. It will be used several times in the sequel.

2.1. Proposition. Let $\pi^{\prime}$ be an irreducible square integrable representation of $S_{q}$ and let $x, y \in(1 / 2) \mathbb{Z}$ such that $x-y \in \mathbb{Z}_{+}$. Let $\rho$ be an $F^{\prime} / F$-selfdual cuspidal unitarizable representation of $G L\left(d_{\rho}\right)$. We assume that $x, y \in \mathbb{Z}$ if and only if $L\left(\rho, R_{d_{\rho}}, s\right)$ has no pole at $s=0$ (see the beginning of this section for the definition of $\left.R_{d_{\rho}}\right)$. Further, suppose that there is an irreducible square integrable representation $\pi$ embedded in the induced representation

$$
\pi \hookrightarrow \nu^{x} \rho \times \cdots \times \nu^{x-i+1} \rho \times \cdots \times \nu^{y} \rho \times \pi^{\prime} .
$$

Then:

(i) If $y>0$, then $2 y-1 \in \operatorname{Jord}_{\rho}\left(\pi^{\prime}\right)$ and

$$
\operatorname{Jord}_{\rho}(\pi)=\left(\operatorname{Jord}_{\rho}\left(\pi^{\prime}\right) \backslash\{(\rho, 2 y-1)\}\right) \cup\{(\rho, 2 x+1)\} .
$$

(ii) If $y \leq 0$, then

$$
\operatorname{Jord}_{\rho}(\pi)=\operatorname{Jord}_{\rho}\left(\pi^{\prime}\right) \cup\{(\rho, 2 x+1),(\rho,-2 y+1)\} .
$$

In particular, $2 x+1$ and $-2 y+1$ are not in $\operatorname{Jord}_{\rho}\left(\pi^{\prime}\right)$.

2.2. Remark. If $x$ does not satisfy the condition regarding the holomorphy of the $L$-function of the proposition, then $(\rho, 2 x+1)$ and $(\rho, 2 y+1)$ are not in $\operatorname{Jord}_{\rho}(\pi)$ and $\operatorname{Jord}_{\rho}\left(\pi^{\prime}\right)$ by the definition of the Jordan blocks. Further, in this case, the embedding (2-8) cannot happen (recall that $\pi^{\prime}$ and $\pi$ are square integrable).

Proof. To compute $\operatorname{Jord}_{\rho}(\pi)$ in terms of $\operatorname{Jord}_{\rho}\left(\pi^{\prime}\right)$, we have to compare Plancherel measures. For $z \in(1 / 2) \mathbb{N}$, we denote by $\mu(z, \pi)(s)$ the meromorphic function (in $s \in \mathbb{C})$, which is the composite of two standard intertwining operators:

$$
\nu^{s} \delta(\rho, z) \rtimes \pi^{\prime} \rightarrow \nu^{-s} \delta(\rho, z) \rtimes \pi^{\prime} \rightarrow \nu^{s} \delta(\rho, z) \rtimes \pi^{\prime}
$$

(here $\delta(\rho, z)$ denotes $\delta\left(\left[\nu^{-(z-1) / 2} \rho, \nu^{(z-1) / 2} \rho\right]\right)$, as before). We are interested in $\mu(z, \pi)(s)$ modulo holomorphic invertible functions of $s$ only. We define $\mu\left(z, \pi^{\prime}\right)(s)$ in an analogous way for $\pi^{\prime}$. By the results of Shahidi, the computation of the 
standard intertwining operators for $G L(d)$ is very well known for arbitrary $d$. This gives the following equality modulo a holomorphic invertible function of $s$ :

$$
\begin{aligned}
& \mu(z, \pi)(s)=\mu\left(z, \pi^{\prime}\right)(s) \\
& L(\delta(\rho, z) \times \rho, s-x) L(\delta(\rho, z) \times \rho, s-y+1)^{-1} \\
& L(\delta(\rho, z) \times \rho, y+s) L(\delta(\rho, z) \times \rho, x+s+1)^{-1} \\
& \quad L(\delta(\rho, z) \times \rho,-s-x) L(\delta(\rho, z) \times \rho,-s-y+1)^{-1} \\
& L(\delta(\rho, z) \times \rho, y-s) L(\delta(\rho, z) \times \rho, x-s+1)^{-1} .
\end{aligned}
$$

The product of $L$-functions is just the product of the normalizing factors in the intertwining operator corresponding to $G L$ 's factors. Usual simplifications are used to get this formula (see [MW], I). By a general result of Harish-Chandra ([W1]) we know that at $s=0, \mu(z, \pi)$ has order 0 or 2 . Moreover, by the definition of $\operatorname{Jord}_{\rho}(\pi)$ :

$$
z \in \operatorname{Jord}_{\rho}(\pi) \Leftrightarrow \operatorname{ord}_{s=0}(\mu(z, \pi))=2 .
$$

We need to look at the different $L$-factors using a general result of [JPSS] (Theorem 8.2 there):

$$
L\left(\delta(\rho, z) \times \rho, s^{\prime}\right)=L\left(\rho \times \rho, s^{\prime}+(z-1) / 2\right) .
$$

For $s^{\prime \prime} \in \mathbb{R}$, the function $L\left(\rho \times \rho, s^{\prime \prime}\right)$ is non-zero and the only pole here is at $s^{\prime \prime}=0$. The pole is simple. It is obvious that at $s=0,(2-9)$ and $(2-10)$ above have the same order.

Now $s=0$ is a pole for $\mu(z, \pi)(s)$ if and only if either it is a pole of $\mu\left(z, \pi^{\prime}\right)(s)$ and not a zero of the factor (2-9) or it is a pole of the factor (2-9). Moreover, if $s=0$ is a pole of the factor (2-9), it cannot be a pole for $\mu\left(z, \pi^{\prime}\right)(s)$ by the result of Harish-Chandra.

To prove the lemma, we first need to observe the following facts:

$$
L(\delta(\rho, z) \times \rho, s-x)
$$

has a (simple) pole at $s=0$ if and only if $x=(z-1) / 2$;

$$
L(\delta(\rho, z) \times \rho, s-y+1)^{-1}
$$

has a (simple) zero at $s=0$ if and only if $y-1=(z-1) / 2$;

$$
L(\delta(\rho, z) \times \rho, y+s)
$$

has a (simple) pole at $s=0$ if and only if $y=-(z-1) / 2$;

$$
L(\delta(\rho, z) \times \rho, x+s+1)^{-1}
$$

has a (simple) zero at $s=0$ if and only if $x+1=-(z-1) / 2$ (this cannot happen since $x+1=-(z-1) / 2$ implies $z=-2 x-1 \leq-2$, which is impossible).

These observations imply that $2 x+1 \in \bar{J}_{\operatorname{ord}}(\pi)$ and $2 x+1 \notin \operatorname{Jord}_{\rho}\left(\pi^{\prime}\right)$. Further, $a^{\prime} \neq 2 x+1$ is an element of $\operatorname{Jord}_{\rho}\left(\pi^{\prime}\right)$ if and only if it is an element of $\operatorname{Jord}_{\rho}(\pi)$, except if $a^{\prime}=-2 y+1$ (which implies $y \leq 0$ ) or $a^{\prime}=2 y-1$ (which implies $y>0)$. In the case $a^{\prime}=-2 y+1$, we have $a^{\prime} \notin \operatorname{Jord}_{\rho}\left(\pi^{\prime}\right)$ but $a^{\prime} \in \operatorname{Jord}_{\rho}(\pi)$. The case $a^{\prime}=-2 y+1$ gives that $a^{\prime} \notin \operatorname{Jord}_{\rho}(\pi)$ and $a^{\prime} \in \operatorname{Jord}_{\rho}\left(\pi^{\prime}\right)$, to avoid a zero of $\mu\left(a^{\prime}, \pi\right)(s)$ at $s=0$. 
2.3. Remark. In this remark we shall suppose that $S_{n}$ is the split group $S p(2 n, F)$ or $S O(2 n+1, F)$.

(i) By [Sh1], the condition (J-1) is equivalent to

$$
a \text { is even if } \nu^{1 / 2} \rho \rtimes 1 \text { reduces and odd otherwise }
$$

$$
\text { (i.e. if } \rho \rtimes 1 \text { or } \nu \rho \rtimes 1 \text { reduces). }
$$

(ii) If $a>1$, then by the fourth section of [T2], (J-1) is equivalent to the fact that

$$
\delta(\rho, a) \rtimes 1 \text { reduces }
$$

(here, 1 denotes the trivial representation of $S_{0}$ ).

\section{General technical lemmas}

In this section we shall collect some facts from representation theory. Most of the results are for classical groups. Some of them are very simple and well known. First we shall recall of the following simple fact.

3.1. Lemma (see [B-Z], 2.4). Let $\sigma$ be an irreducible cuspidal subquotient of a Jacquet module $r_{M}^{G}(\pi)$ of an irreducible representation $\pi$ of a connected reductive $p$-adic group $G$ with respect to a parabolic subgroup $P=M N$ of $G$. Then

$$
\pi \hookrightarrow \operatorname{Ind}_{P}^{G}(\sigma) .
$$

Proof. By Frobenius reciprocity, we have to prove that $\sigma$ is a quotient of the Jacquet module of $\pi$ relative to $P$. This Jacquet module, denoted $\pi_{P}$, is a representation of finite length of $M$. Bernstein has proved that one can decompose such a representation into a direct sum of representations:

$$
\pi_{P}=\pi_{P}[\sigma] \oplus \pi_{P}^{\prime},
$$

where all irreducible subquotients of $\pi_{P}[\sigma]$ are isomorphic to $\sigma$ and no irreducible subquotient of $\pi_{P}^{\prime}$ is isomorphic to $\sigma$. By hypothesis, $\pi_{P}[\sigma]$ is non-zero. From this it follows that $\sigma$ is a quotient of $\pi_{P}[\sigma]$ and of $\pi_{P}$. The lemma is proved.

3.2. Lemma. Let $\pi$ be an irreducible representation of a reductive p-adic group and let $P=M N$ be a parabolic subgroup of $G$. Suppose that $M$ is a direct product of two reductive subgroups $M_{1}$ and $M_{2}$. Let $\tau_{1}$ be an irreducible representation of $M_{1}$ and let $\tau_{2}$ be a representation of $M_{2}$. Suppose

$$
\pi \hookrightarrow \operatorname{Ind}_{P}^{G}\left(\tau_{1} \otimes \tau_{2}\right) .
$$

Then there exists an irreducible representation $\tau_{2}^{\prime}$ such that

$$
\pi \hookrightarrow \operatorname{Ind}_{P}^{G}\left(\tau_{1} \otimes \tau_{2}^{\prime}\right) .
$$

Proof. First note that there is a non-zero intertwining morphism from the Jacquet module $r_{M}^{G}(\pi)$ of $\pi$ (with respect to $P=M N$ ) into $\tau_{1} \otimes \tau_{2}$. The image $X$ has a finite length as a representation of $M$. Therefore, $X$ has an irreducible quotient, say $\tau_{1}^{\prime} \otimes \tau_{2}^{\prime}$. To see the lemma, it is enough to show $\tau_{1}^{\prime} \cong \tau_{1}$.

Now $\tau_{1} \otimes \tau_{2}$ (resp. $\tau_{1}^{\prime} \otimes \tau_{2}^{\prime}$ ) is, as a representation of $M_{1}$, a sum of copies of $\tau_{1}\left(\right.$ resp. $\left.\tau_{1}^{\prime}\right)$. From the existence of a non-zero $M_{1}$-intertwining morphism from the subspace $X$ of $\tau_{1} \otimes \tau_{2}$ into $\tau_{1}^{\prime} \otimes \tau_{2}^{\prime}$, we get $\tau_{1}^{\prime} \cong \tau_{1}$ (since $X$ is a semi-simple representation of $M_{1}$, and it is isomorphic to a direct sum of copies of $\tau_{1}$ ). 
3.3. Lemma. Let $\pi$ be an irreducible representation of $S_{q}$. The following three sets of irreducible cuspidal representations $\tau$ of general linear groups coincide.

(1) The set of all $\tau$ for which there exists an irreducible subquotient $\sigma \otimes \pi_{\text {cusp }}$ of $s_{G L}(\pi)$, such that $\tau$ is in the support of $\sigma$ (the support is defined in Proposition 1.10 of $[\mathrm{Z}]$ ).

(2) The set of all $\tau$ such that there exists an irreducible cuspidal subquotient $\rho_{1} \otimes \cdots \otimes \rho_{k} \otimes \sigma$ of a standard Jacquet module of $\pi$ and index $i$ such that $\tau \cong$ $\rho_{i}$ (standard Jacquet module means that it is a Jacquet module with respect to a standard parabolic subgroup).

(3) The set of all $\tau$ such that there exist irreducible cuspidal representations $\rho_{1}, \ldots, \rho_{k}$ and $\sigma$ of general linear groups and of some $S_{q^{\prime}}$ respectively, and an index $i$ such that

$$
\pi \hookrightarrow \rho_{1} \times \cdots \times \rho_{k} \rtimes \sigma
$$

and $\tau \cong \rho_{i}$.

Irreducible cuspidal representations of general linear groups characterized by one of the above descriptions will be called factors of $\pi$.

Proof. For $i=1,2,3$, denote the set described in (i) by $X_{i}$. Proposition 1.10 of $[\mathrm{Z}]$ and induction in stages imply $X_{1} \subseteq X_{2}$. The transitivity of Jacquet modules and the definition of the support in Proposition 1.10 of [Z] imply $X_{2} \subseteq X_{1}$. Frobenius reciprocity implies $X_{3} \subseteq X_{2}$. At the end, $X_{2} \subseteq X_{1}$ follows from Lemma 3.1.

The following lemma is essentially a claim about the representations of general linear groups.

3.4. Lemma. Let $\pi$ be an irreducible representation of $S_{q}$ and let $\tau$ be a factor of $\pi$ such that $\nu^{-1} \tau$ is not a factor of $\pi$.

(i) Then there exists $z \in \mathbb{Z}_{+}$and an irreducible representation $\sigma$ of some $S_{q^{\prime}}$, $q^{\prime}<q$, such that

$$
\pi \hookrightarrow \delta\left(\left[\tau, \nu^{z} \tau\right]\right) \rtimes \sigma .
$$

(ii) Let $\rho_{1}^{\prime} \otimes \rho_{2}^{\prime} \otimes \cdots \otimes \rho_{l}^{\prime} \otimes \pi_{\text {cusp }}$ be an irreducible cuspidal subquotient of a Jacquet module of $\pi$, such that $\tau \cong \rho_{j^{\prime}}^{\prime}$. Suppose that there exists $j \in \mathbb{Z}_{+}$such that $\nu^{j+1} \tau \neq \rho_{i}^{\prime}$ for all $i<j^{\prime}$. Then one can find an embedding in (i) for which $z \leq j$.

(iii) Let $\rho_{1}^{\prime} \otimes \rho_{2}^{\prime} \otimes \cdots \otimes \rho_{l}^{\prime} \otimes \pi_{\text {cusp }}$ be an irreducible cuspidal subquotient of a Jacquet module of $\pi$. Suppose that $\tau \cong \rho_{m}^{\prime}$ for some $m$. Then there exist $k \in \mathbb{Z}_{+}$, $k \leq m-1$, and irreducible cuspidal representations $\rho_{i}, k+2 \leq i \leq l$, such that

$$
\begin{aligned}
& \pi \hookrightarrow \delta\left(\left[\tau, \nu^{k} \tau\right]\right) \times \rho_{k+2} \times \cdots \times \rho_{l} \rtimes \pi_{c u s p} ; \\
& \rho_{i}^{\prime} \cong \rho_{i} \text { for } m+1 \leq i \leq l ; \\
& \nu^{k} \tau, \nu^{k-1} \tau, \ldots, \tau, \rho_{k+2}, \rho_{k+3}, \ldots, \rho_{m} \text { is a permutation of } \rho_{1}^{\prime}, \rho_{2}^{\prime}, \ldots, \rho_{m}^{\prime} .
\end{aligned}
$$

Proof. Obviously, (ii) implies (i). Now we shall show that (iii) implies (ii). Suppose that we have proved (iii). Applying (iii) to (ii) for $m=j^{\prime}$, we get $\pi \hookrightarrow \delta\left(\left[\tau, \nu^{k} \tau\right]\right) \times$ $\rho_{k+2} \times \cdots \times \rho_{l} \rtimes \pi_{c u s p}$, where (ii) and (iii) imply $k \leq j$. Now Lemma 3.2 implies (ii).

It remains to prove (iii). We shall do it now.

Look at all possible embeddings like

$$
\pi \hookrightarrow\left(\prod_{j=1}^{l} \rho_{j}\right) \rtimes \pi_{c u s p}
$$


such that (2) and (3) hold. By Lemma 3.1, there exists at least one such embedding. For such an embedding, we know that $\rho_{i} \cong \tau$ for at least one $i \leq j$. Among all such embeddings, choose one with minimal possible index $i$ such that $\rho_{i} \cong \tau$. Clearly, $i \leq m$. The condition of the lemma implies

$$
\rho_{j} \not \nu^{-1} \rho_{i} \text { for } 1 \leq j \leq i-1 \text {. }
$$

Denote

$$
\pi^{\prime}=\left(\prod_{j=i+1}^{l} \rho_{j}\right) \rtimes \pi_{c u s p} \quad \text { and } \quad \tau=\left(\bigotimes_{j=i+1}^{l} \rho_{j}\right) \otimes \pi_{c u s p} .
$$

Let $j^{\prime} \in\{0,1, \ldots, i-1\}$ be maximal such that

$$
\rho_{i-j}=\nu^{j} \rho_{i} \text { for } j=0,1, \ldots, j^{\prime},
$$

and

$$
\pi \hookrightarrow\left(\prod_{j=1}^{i-j^{\prime}-1} \rho_{j}\right) \times \delta\left(\left[\rho_{i}, \nu^{j^{\prime}} \rho_{i}\right]\right) \rtimes \pi^{\prime} .
$$

Clearly, there is at least one such $j^{\prime} \geq 0$ satisfying (3-4) and (3-5) (it is $j^{\prime}=0$ ). We shall prove $j^{\prime}=i-1$. This will complete the proof of the lemma.

Suppose $j^{\prime}<i-1$. Look at $\rho_{i-j^{\prime}-1}$. If $\rho_{i-j^{\prime}-1} \times \delta\left(\left[\rho_{i}, \nu^{j^{\prime}} \rho_{i}\right]\right)$ is irreducible, then $\rho_{i-j^{\prime}-1} \times \delta\left(\left[\rho_{i}, \nu^{j^{\prime}} \rho_{i}\right]\right) \cong \delta\left(\left[\rho_{i}, \nu^{j^{\prime}} \rho_{i}\right]\right) \times \rho_{i-j^{\prime}-1}$. This would imply the existence of an embedding of $\pi$ like in (3-2), with $\rho_{i}$ at the $(i-1)$-th place. Therefore, $\rho_{i-j^{\prime}-1} \times \delta\left(\left[\rho_{i}, \nu^{j^{\prime}} \rho_{i}\right]\right)$ reduces. There are two possibilities for that. The possibility $\rho_{i-j^{\prime}-1} \cong \nu^{-1} \rho_{i}$ cannot happen by the assumption of the lemma. Thus,

$$
\rho_{i-j^{\prime}-1}=\nu^{j^{\prime}+1} \rho_{i} .
$$

This implies that (3-4) holds for $j^{\prime}+1$. Further, (3-5), (3-6) and the Frobenius reciprocity imply that we have a non-zero intertwining $\phi$ from the corresponding Jacquet module $r_{M}^{S_{q}}(\pi)$ of $\pi$ into

$$
\left(\bigotimes_{j=1}^{i-j^{\prime}-2} \rho_{j}\right) \otimes \nu^{j^{\prime}+1} \rho_{i} \times \delta\left(\left[\rho_{i}, \nu^{j^{\prime}} \rho_{i}\right]\right) \otimes \tau
$$

Note that (3-7) is a length two representation with a unique irreducible subrepresentation, which is $\left(\bigotimes_{j=1}^{i-j^{\prime}-2} \rho_{j}\right) \otimes \delta\left(\left[\rho_{i}, \nu^{j^{\prime}+1} \rho_{i}\right]\right) \otimes \tau$. Since $j^{\prime}$ is maximal, the image of $\phi$ cannot be this irreducible subrepresentation (otherwise, Frobenius reciprocity would imply that (3-5) holds also for $j^{\prime}+1$, and this would contradict the assumption that $j^{\prime}$ is maximal, since we have seen already that (3-4) holds for $\left.j^{\prime}+1\right)$. Therefore, the image is the whole (3-7). From this (and transitivity of Jacquet modules) we conclude that

$$
\left(\bigotimes_{j=1}^{i-j^{\prime}-2} \rho_{j}\right) \otimes \delta\left(\left[\rho_{i}, \nu^{j^{\prime}} \rho_{i}\right]\right) \otimes \nu^{j^{\prime}+1} \rho_{i} \otimes \tau
$$

is a subquotient of a corresponding Jacquet module of $\pi$. Now Lemma 3.1 implies that $\rho_{i}$ can show up in embeddings like (3-2) at the $(i-1)$-th place, which contradicts our choice of $i$. This contradiction ends the proof that $j^{\prime}=i-1$. 
Now we shall recall the notion of a strongly positive (or strictly positive) irreducible representation of $S_{q}$, defined in $\mathrm{M} 2$. One says that $\pi$ is strongly positive if for any factor $\tau$ of $\pi$ we have

$$
e(\tau)>0 .
$$

Casselman's square integrability criterion implies that each strongly positive irreducible representation is square integrable.

In this paper, we shall use the following several times.

3.5. Lemma. Let $\pi$ and $\tau$ be irreducible representations of $S_{q}$ and $S_{q^{\prime}}$ respectively. Suppose that $\tau$ is tempered (resp. square integrable) and $q>q^{\prime}$. Let $\gamma$ be a representation of $G L\left(q-q^{\prime}, F^{\prime}\right)$ of finite length. Suppose

$$
s_{G L}(\pi) \leq \gamma \times s_{G L}(\tau),
$$

and suppose that for each irreducible cuspidal subquotient $\rho_{1} \otimes \cdots \otimes \rho_{k}$ of a standard Jacquet module (with respect to the subgroup of upper triangular matrices; see $[\mathbf{Z}]$ ) of $\gamma$, we have

$$
\begin{array}{r}
\sum_{i=1}^{j} e\left(\rho_{i}\right) d_{\rho_{i}} \geq 0 \\
\text { (resp. } \left.\sum_{i=1}^{j} e\left(\rho_{i}\right) d_{\rho_{i}}>0\right)
\end{array}
$$

for all $j=1, \ldots, k$ (recall that $d_{\rho_{i}}$ is defined by the condition that $\rho_{i}$ is a representation of $\left.G L\left(d_{\rho_{i}}, F^{\prime}\right)\right)$. Then $\pi$ is tempered (resp. square integrable).

Proof. First note $\pi_{\text {cusp }}=\tau_{\text {cusp }}$. Further, (3-8) implies that for each irreducible cuspidal subquotient

$$
\rho_{1} \otimes \cdots \otimes \rho_{n} \otimes \pi_{c u s p}
$$

of a standard Jacquet module of $\pi$, there exists a partition of $\{1, \ldots, n\}$ into two subsets

$$
i_{1}<i_{2}<\cdots<i_{l} \text { and } j_{1}<j_{2}<\cdots<j_{m} \text {, }
$$

such that

$$
\rho_{i_{1}} \otimes \cdots \otimes \rho_{i_{l}}
$$

is a subquotient of a standard Jacquet module of $\gamma$ and

$$
\rho_{j_{1}} \otimes \cdots \otimes \rho_{i_{m}} \otimes \pi_{\text {cusp }}
$$

is a subquotient of a standard Jacquet module of $\tau$. Then transitivity of Jacquet modules implies that $\rho_{j_{1}} \otimes \cdots \otimes \rho_{i_{m}} \otimes \pi_{\text {cusp }}$ is a subquotient of a standard Jacquet module of $s_{G L}(\tau)$.

By Casselman's square integrability criterion and the description of the positive simple roots in the case of classical groups, $\pi$ is square integrable if and only if for each subquotient (3-11) we have

$$
\sum_{i=1}^{j} e\left(\rho_{i}\right) d_{\rho_{i}}>0 \quad \text { for every } j=1, \ldots, n .
$$

For details of Casselman's square integrability criterion in the symplectic case one can see section 6 of [T6] (the case for $S O(2 k+1, F)$ goes completely in the same 
way; see [T5] for roots in this case). Suppose that $\tau$ is square integrable and $\gamma$ satisfies (3-10). Then (3-10), square integrability criterion (3-12) applied to $\tau$, and the above description of the subquotients (3-11) in terms of Jacquet modules of $\gamma$ and $\tau$ imply that $\pi$ is square integrable. In the same way one proves the claim of the lemma in the tempered case.

Now we shall write a direct consequence of 5.1.2 from [M2].

3.6. Lemma. Suppose that $\pi$ is an irreducible square integrable representation of $S_{q}$. Let $\nu^{x} \rho \otimes \tau$ be an irreducible subquotient of a standard Jacquet module of $\pi$, where $\rho$ is an irreducible $F^{\prime} / F$-selfdual cuspidal representation of $G L\left(p, F^{\prime}\right), x \in \mathbb{R}$, and $\tau$ is an irreducible representation of $S_{q^{\prime}}$. Then

$$
(\rho, 2 x+1) \in \operatorname{Jord}(\pi) .
$$

Proof. Take irreducible cuspidal representations $\rho_{1}, \ldots, \rho_{l}$ and $\sigma$ of general linear groups and (some) $S_{q^{\prime \prime}}$ respectively, such that

$$
\tau \hookrightarrow \rho_{1} \times \cdots \times \rho_{l} \rtimes \sigma .
$$

By the transitivity of Jacquet modules (and the Frobenius reciprocity), $\nu^{x} \rho \otimes \rho_{1} \times$ $\cdots \otimes \rho_{l} \otimes \sigma$ is a(n irreducible cuspidal) subquotient of a standard Jacquet module of $\pi$. Lemma 3.1 implies

$$
\pi \hookrightarrow \nu^{x} \rho \times \rho_{1} \times \cdots \times \rho_{l} \rtimes \sigma .
$$

Lemma 3.2 implies the existence of an irreducible representation $\tau^{\prime}$ such that

$$
\pi \hookrightarrow \nu^{x} \rho \rtimes \tau^{\prime} .
$$

Now 5.1.2 of [M2] implies the lemma.

\section{ON IRREDUCIBLE SUBREPRESENTATIONS}

In this section we shall present some facts related to irreducible subrepresentations and their uniqueness. These facts shall be used frequently later.

4.1. Lemma. Let $n$ be a non-zero integer, let $\rho$ be an irreducible cuspidal $F^{\prime} / F$ selfdual representation of a general linear group and let $\pi_{c u s p}$ be an irreducible cuspidal representation of $S_{q}$. Suppose that $\alpha, \beta:\{1, \ldots, n\} \rightarrow \mathbb{R}$ are functions which satisfy

$$
\begin{aligned}
& \beta(i)-\alpha(i) \in \mathbb{Z}_{+} \text {for all } i, \\
& \alpha(i)>0 \text { for all } i, \quad \text { and } \\
& \beta(1)<\beta(2)<\cdots<\beta(n) .
\end{aligned}
$$

Then the representation

$$
\left(\prod_{i=1}^{n} \delta\left(\left[\nu^{\alpha(i)} \rho, \nu^{\beta(i)} \rho\right]\right)\right) \rtimes \pi_{c u s p}
$$

has a unique irreducible subrepresentation.

Proof. For the proof of the lemma (by Frobenius reciprocity) it is enough to show that the multiplicity of

$$
\left(\bigotimes_{i=1}^{n} \delta\left(\left[\nu^{\alpha(i)} \rho, \nu^{\beta(i)} \rho\right]\right)\right) \otimes \pi_{c u s p}
$$


in the corresponding standard Jacquet module of $\left(\prod_{i=1}^{n} \delta\left(\left[\nu^{\alpha(i)} \rho, \nu^{\beta(i)} \rho\right]\right)\right) \rtimes \pi_{\text {cusp }}$ is one.

For a moment suppose that $\tau_{1}, \tau_{2}, \ldots, \tau_{\ell}$ are finite length representations of general linear groups. Then (1-2), applied (several times) to $\tau_{1} \times \tau_{2} \times \cdots \times \tau_{\ell} \rtimes \pi_{\text {cusp }}$, gives

$$
\begin{aligned}
& \text { s.s. }\left(s_{G L}\left(\tau_{1} \times \tau_{2} \times \cdots \times \tau_{\ell} \rtimes \pi_{\text {cusp }}\right)\right) \\
& \quad=M_{G L}^{*}\left(\tau_{1}\right) \times M_{G L}^{*}\left(\tau_{2}\right) \times \cdots \times M_{G L}^{*}\left(\tau_{\ell}\right) \otimes \pi_{\text {cusp }}
\end{aligned}
$$

since $\pi_{\text {cusp }}$ is cuspidal. Now applying this observation to $\left(\prod_{i=1}^{n} \delta\left(\left[\nu^{\alpha(i)} \rho, \nu^{\beta(i)} \rho\right]\right)\right) \rtimes$ $\pi_{\text {cusp }}$ and using formula (1-4) we get

$$
\begin{aligned}
\text { s.s. }\left(s_{G L}\right. & \left.\left(\left(\prod_{i=1}^{n} \delta\left(\left[\nu^{\alpha(i)} \rho, \nu^{\beta(i)} \rho\right]\right)\right) \rtimes \pi_{c u s p}\right)\right) \\
& =\left(\prod_{i=1}^{n}\left(\sum_{j_{i}=\alpha(i)}^{\beta(i)+1} \delta\left(\left[\nu^{j_{i}} \rho, \nu^{\beta(i)} \rho\right]\right) \times \delta\left(\left[\nu^{-j_{i}+1} \rho, \nu^{-\alpha(i)} \rho\right]\right)\right)\right) \otimes \pi_{\text {cusp }} .
\end{aligned}
$$

The above formula also follows from an explicit description of Jacquet modules of parabolically induced representations in 1.3 of [B-Z] (after some computation). Now (4-2) and the above formula imply that it is enough to see that the multiplicity of (4-4) in a corresponding Jacquet module of $\left(\prod_{i=1}^{n} \delta\left(\left[\nu^{\alpha(i)} \rho, \nu^{\beta(i)} \rho\right]\right)\right) \otimes \pi_{\text {cusp }}$ is one (since to get (4-4) for a subquotient of a corresponding Jacquet module of a term in the above formula, one needs to take $j_{i}=\alpha(i)$ for all $i$, by (4-2)). To see this, it is enough to show that the multiplicity of

$$
\bigotimes_{i=1}^{n} \delta\left(\left[\nu^{\alpha(i)} \rho, \nu^{\beta(i)} \rho\right]\right)
$$

in a corresponding Jacquet module of

$$
\prod_{i=1}^{n} \delta\left(\left[\nu^{\alpha(i)} \rho, \nu^{\beta(i)} \rho\right]\right)
$$

is one.

Note that the multiplicativity of $m^{*}$ (recall that $R$ is a Hopf algebra) implies

$$
\begin{aligned}
m^{*}\left(\prod_{i=1}^{n} \delta\left(\left[\nu^{\alpha(i)} \rho, \nu^{\beta(i)} \rho\right]\right)\right) & \\
& =\prod_{i=1}^{n}\left(\sum_{j_{i}=\alpha(i)-1}^{\beta(i)} \delta\left(\left[\nu^{j_{i}+1} \rho, \nu^{\beta(i)} \rho\right]\right) \otimes \delta\left(\left[\nu^{\alpha(i)} \rho, \nu^{j_{i}} \rho\right]\right)\right) .
\end{aligned}
$$

Let $\prod_{i=1}^{n} \delta\left(\left[\nu^{\alpha(i)} \rho, \nu^{\beta(i)} \rho\right]\right)$ and $\delta\left(\left[\nu^{\alpha(i)} \rho, \nu^{\beta(i)} \rho\right]\right)$ be representations of $G L\left(p^{\prime}, F^{\prime}\right)$ and $G L\left(k_{i}, F^{\prime}\right)$, respectively.

Now we shall show that the multiplicity of $\bigotimes_{i=1}^{n} \delta\left(\left[\nu^{\alpha(i)} \rho, \nu^{\beta(i)} \rho\right]\right)$ in a corresponding Jacquet module of $\prod_{i=1}^{n} \delta\left(\left[\nu^{\alpha(i)} \rho, \nu^{\beta(i)} \rho\right]\right)$ is one, by induction with respect to $n$. For $n=1$ the claim obviously holds. Suppose $n>1$.

We shall compute the multiplicity of $\bigotimes_{i=1}^{n} \delta\left(\left[\nu^{\alpha(i)} \rho, \nu^{\beta(i)} \rho\right]\right)$ in a corresponding Jacquet module of $\prod_{i=1}^{n} \delta\left(\left[\nu^{\alpha(i)} \rho, \nu^{\beta(i)} \rho\right]\right)$ using (4-6) and transitivity of Jacquet modules. First we consider the Jacquet module with respect to $G L\left(p^{\prime}-k_{n}, F^{\prime}\right) \times$ 
$G L\left(k_{n}, F^{\prime}\right)$. To get $\bigotimes_{i=1}^{n} \delta\left(\left[\nu^{\alpha(i)} \rho, \nu^{\beta(i)} \rho\right]\right)$ for a subquotient of a corresponding Jacquet module of a term in (4-6), we must take $j_{n}=\beta(n)$ (note that $\nu^{\beta(n)} \rho$ shows up in the support of the last tensor factors of $\bigotimes_{i=1}^{n} \delta\left(\left[\nu^{\alpha(i)} \rho, \nu^{\beta(i)} \rho\right]\right)$, and then use (4-3)). Therefore, $\delta\left(\left[\nu^{\alpha(n)} \rho, \nu^{\beta(n)} \rho\right]\right)$ shows up on the right-hand side of $\otimes$. Since we are considering the Jacquet module with respect to $G L\left(p^{\prime}-k_{n}, F^{\prime}\right) \times G L\left(k_{n}, F^{\prime}\right)$, we must have $j_{i}=\alpha(i)-1$ for $1 \leq i \leq n-1$. Now applying the inductive assumption in the case of $n-1$, we get the claim for $n$.

The following evident fact will be used a number of times.

4.2. Remark. Let $\pi$ and $\pi^{\prime}$ be representations of finite length. Suppose that $\pi$ has at most $n$ irreducible subrepresentations. Let $\pi^{\prime} \hookrightarrow \pi$. Then $\pi^{\prime}$ also has at most $n$ irreducible subrepresentations.

The following lemma holds in a much bigger generality.

4.3. Lemma. Let $\pi$ be an irreducible square integrable representation of $S_{q}$ and let $\rho$ be an irreducible $F^{\prime} / F$-selfdual cuspidal representation of $G L\left(p, F^{\prime}\right)$. Let $\alpha, \beta \in$ $(1 / 2) \mathbb{Z}_{+}$. Then:

(i) The multiplicity of $\delta\left(\left[\nu^{-\alpha} \rho, \nu^{\alpha} \rho\right]\right) \otimes \pi$ in $\mu^{*}\left(\delta\left(\left[\nu^{-\alpha} \rho, \nu^{\alpha} \rho\right]\right) \rtimes \pi\right)$ is 2 .

(ii) Suppose $\beta>\alpha$. Then the multiplicity of $\delta\left(\left[\nu^{-\beta} \rho, \nu^{\beta} \rho\right]\right) \otimes \delta\left(\left[\nu^{-\alpha} \rho, \nu^{\alpha} \rho\right]\right) \otimes \pi$ in a corresponding Jacquet module of $\delta\left(\left[\nu^{-\beta} \rho, \nu^{\beta} \rho\right]\right) \times \delta\left(\left[\nu^{-\alpha} \rho, \nu^{\alpha} \rho\right]\right) \rtimes \pi$ is 4 .

Proof. We have

$$
\begin{aligned}
& \mu^{*}\left(\delta\left(\left[\nu^{-\alpha} \rho, \nu^{\alpha} \rho\right]\right) \rtimes \pi\right) \\
= & \left(\sum_{i=-\alpha-1}^{\alpha} \sum_{j=i}^{\alpha} \delta\left(\left[\nu^{-i} \rho, \nu^{\alpha} \rho\right]\right) \times \delta\left(\left[\nu^{j+1} \rho, \nu^{\alpha} \rho\right]\right) \otimes \delta\left(\left[\nu^{i+1} \rho, \nu^{j} \rho\right]\right)\right) \rtimes \mu^{*}(\pi) .
\end{aligned}
$$

If $i=j$ in (4-7), then we get directly that $\delta\left(\left[\nu^{-\alpha} \rho, \nu^{\alpha} \rho\right]\right) \otimes \pi$ can be a subquotient only if $i=-\alpha-1$ and $\alpha$. In each term the multiplicity is one (each of these terms is just $\left.\delta\left(\left[\nu^{-\alpha} \rho, \nu^{\alpha} \rho\right]\right) \otimes \pi\right)$.

Suppose that we can get $\delta\left(\left[\nu^{-\alpha} \rho, \nu^{\alpha} \rho\right]\right) \otimes \pi$ from a term corresponding to $i<j$. Then $-\alpha-1 \leq i<j$ implies $j+1>-\alpha$. Also $-i>-\alpha$ (if $-i=-\alpha$, then $i=\alpha$ and thus $j=i=\alpha$ which contradicts $i<j$ ). Thus $\nu^{-\alpha} \rho$ must come from $\mu^{*}(\pi)$. From the above discussion and (4-7) we get that $\delta\left(\left[\nu^{-\alpha} \rho, \nu^{\alpha-k} \rho\right]\right) \otimes \tau \leq \mu^{*}(\pi)$ for some $k \in \mathbb{Z}_{+}, k \leq 2 \alpha$. Casselman's square integrability criterion implies that $\pi$ is not square integrable. This contradiction completes the proof of (i).

Suppose $\alpha<\beta$. Write

$$
\begin{aligned}
& \mu^{*}\left(\delta\left(\left[\nu^{-\beta} \rho, \nu^{\beta} \rho\right]\right) \times \delta\left(\left[\nu^{-\alpha} \rho, \nu^{\alpha} \rho\right]\right) \rtimes \pi\right) \\
& =\left(\sum_{i=-\beta-1}^{\beta} \sum_{j=i}^{\beta} \delta\left(\left[\nu^{-i} \rho, \nu^{\beta} \rho\right]\right) \times \delta\left(\left[\nu^{j+1} \rho, \nu^{\beta} \rho\right]\right) \otimes \delta\left(\left[\nu^{i+1} \rho, \nu^{j} \rho\right]\right)\right) \\
& \times M^{*}\left(\delta\left(\left[\nu^{-\alpha} \rho, \nu^{\alpha} \rho\right]\right)\right) \rtimes \mu^{*}(\pi) .
\end{aligned}
$$

We analyze when one can get $\delta\left(\left[\nu^{-\beta} \rho, \nu^{\beta} \rho\right]\right) \otimes \tau$ for a subquotient of (4-8) (where $\tau$ is an arbitrary irreducible representation). One can get it for $i=j=-\beta-1$ or $\beta$, and these are the only cases when one can get it if $i=j$. Then the corresponding term in the above sum is $\delta\left(\left[\nu^{-\beta} \rho, \nu^{\beta} \rho\right]\right) \otimes M^{*}\left(\delta\left(\left[\nu^{-\alpha} \rho, \nu^{\alpha} \rho\right]\right)\right) \rtimes \mu^{*}(\pi)=$ $\delta\left(\left[\nu^{-\beta} \rho, \nu^{\beta} \rho\right]\right) \otimes \mu^{*}\left(\delta\left(\left[\nu^{-\alpha} \rho, \nu^{\alpha} \rho\right]\right) \rtimes \pi\right)$. Now (i) tells us that the multiplicity of $\delta\left(\left[\nu^{-\beta} \rho, \nu^{\beta} \rho\right]\right) \otimes \delta\left(\left[\nu^{-\alpha} \rho, \nu^{\alpha} \rho\right]\right) \otimes \pi$ in each of these two terms is two. Therefore, to 
prove (ii), one needs to show that one cannot get $\delta\left(\left[\nu^{-\beta} \rho, \nu^{\beta} \rho\right]\right) \otimes \tau$ for a subquotient of (4-8) if $i<j$.

Suppose that we can get it for some $i<j$. We shall now have similar arguments as in the proof of (i). To get $\delta\left(\left[\nu^{-\beta} \rho, \nu^{\beta} \rho\right]\right) \otimes \tau$, one needs to get $\nu^{-\beta} \rho$ on the left-hand side of $\otimes$. Note that $\nu^{-\beta} \rho$ cannot come from $M^{*}\left(\delta\left(\left[\nu^{-\alpha} \rho, \nu^{\alpha} \rho\right]\right)\right)$ since $\alpha<\beta$. Suppose now that $M^{*}\left(\delta\left(\left[\nu^{-\alpha} \rho, \nu^{\alpha} \rho\right]\right)\right)$ gives a non-trivial contribution to the left-hand side of $\otimes$ of the term where $\delta\left(\left[\nu^{-\beta} \rho, \nu^{\beta} \rho\right]\right) \otimes \tau$ is a subquotient. Then a short discussion gives $\delta\left(\left[\nu^{-\beta} \rho, \nu^{\beta-k} \rho\right]\right) \otimes \tau \leq \mu^{*}(\pi)$ for some $k \in \mathbb{N}, k \leq 2 \beta$. This contradicts the square integrability of $\pi$. Thus, $M^{*}\left(\delta\left(\left[\nu^{-\alpha} \rho, \nu^{\alpha} \rho\right]\right)\right)$ does not give a non-trivial contribution to the left-hand side of $\otimes$. Now one can repeat the argument from (i) without change, and get that we cannot get $\delta\left(\left[\nu^{-\beta} \rho, \nu^{\beta} \rho\right]\right) \otimes \tau$ for a subquotient if $i<j$. This completes the proof.

The following lemma (which holds in a much bigger generality) points out a well-known property of the Langlands classification. We state it in the setting in which we shall use the property later.

4.4. Lemma. Suppose that $\tau$ is an irreducible tempered representation of $S_{q}$ and suppose that $\rho$ is an irreducible cuspidal $F^{\prime} / F$-selfdual representation of a general linear group $G L(p)$. Let $x, y \in \mathbb{R}$ be such that $y-x \in \mathbb{Z}_{+}$and $x+y>0$. Then the representation

$$
\delta\left(\left[\nu^{x} \rho, \nu^{y} \rho\right]\right) \rtimes \tau
$$

has a unique irreducible quotient, which will be denoted by $\pi$. The multiplicity of $\delta\left(\left[\nu^{-y} \rho, \nu^{-x} \rho\right]\right) \otimes \tau$ in $\mu^{*}\left(\delta\left(\left[\nu^{x} \rho, \nu^{y} \rho\right]\right) \rtimes \tau\right)$ and $\mu^{*}(\pi)$ is one.

Proof. It is a well-known property of the Langlands classification that the multiplicity of $\delta\left(\left[\nu^{-y} \rho, \nu^{-x} \rho\right]\right) \otimes \tau$ in $\mu^{*}\left(\delta\left(\left[\nu^{x} \rho, \nu^{y} \rho\right]\right) \rtimes \tau\right)$ is one (this implies the uniqueness of the irreducible quotient). One can also get this fact easily by using formula (1-1) (and (1-3), where we allow $a_{-}$in (1-3) also to be negative). Further, $\pi \hookrightarrow \delta\left(\left[\nu^{-y} \rho, \nu^{-x} \rho\right]\right) \rtimes \tau$ (this follows from the fact that $\pi$ is an image of the long intertwining operator in the Langlands classification). Frobenius reciprocity now implies that the multiplicity of $\delta\left(\left[\nu^{-y} \rho, \nu^{-x} \rho\right]\right) \otimes \tau$ in $\mu^{*}(\pi)$ is one.

4.5. Lemma. Let $\pi$ be an irreducible square integrable representation of $S_{q}$ and let $\rho$ be an irreducible $F^{\prime} / F$-selfdual cuspidal representation of $G L\left(p, F^{\prime}\right)$. Fix a $\in \mathbb{Z}_{+}$ and fix positive numbers $\alpha_{1}, \ldots, \alpha_{k}$. Suppose

(i) $\alpha_{i}>(a-1) / 2$ for all $i=1, \ldots, k$, or $\alpha_{i}<(a-1) / 2$ for all $i=1, \ldots, k$;

(ii) $\alpha_{i} \neq \alpha_{j}$ for $i \neq j$ in $\{1, \ldots, k\}$;

(iii) $\left(\rho, 2 \alpha_{i}+1\right) \notin \operatorname{Jord}(\pi)$ for $i=1, \ldots, k$.

Let $T$ be any irreducible subrepresentation of $\delta\left(\left[\nu^{-(a-1) / 2} \rho, \nu^{(a-1) / 2} \rho\right]\right) \rtimes \pi$ (this is a unitarizable representation $)$. Then the multiplicity of $\left(\otimes_{i=1}^{k} \nu^{\alpha_{i}} \rho\right) \otimes T$ in the standard Jacquet modules of

$$
\left(\prod_{i=1}^{k} \nu^{\alpha_{i}} \rho\right) \rtimes T \quad \text { and } \quad\left(\prod_{i=1}^{k} \nu^{\alpha_{i}} \rho\right) \times \delta\left(\left[\nu^{-(a-1) / 2} \rho, \nu^{(a-1) / 2} \rho\right]\right) \rtimes \pi
$$

is one. In particular, $\left(\prod_{i=1}^{k} \nu^{\alpha_{i}} \rho\right) \rtimes T$ contains a unique irreducible subrepresentation. 
Further, the multiplicity of $\left(\bigotimes_{i=1}^{k} \nu^{\alpha_{i}} \rho\right) \otimes \delta\left(\left[\nu^{-(a-1) / 2} \rho, \nu^{(a-1) / 2} \rho\right]\right) \otimes \pi$ in a corresponding standard Jacquet module of $\left(\prod_{i=1}^{k} \nu^{\alpha_{i}} \rho\right) \times \delta\left(\left[\nu^{-(a-1) / 2} \rho, \nu^{(a-1) / 2} \rho\right]\right) \rtimes \pi$ is two, and the last representation has at most two irreducible subrepresentations. If we have two irreducible subrepresentations, they are not isomorphic.

Proof. Frobenius reciprocity implies that the multiplicity of $\left(\otimes_{i=1}^{k} \nu^{\alpha_{i}} \rho\right) \otimes T$ in a corresponding Jacquet module of $\left(\prod_{i=1}^{k} \nu^{\alpha_{i}} \rho\right) \rtimes T$ is at least one. Now using (1-1) and (1-3) we obtain

$$
\begin{aligned}
& \mu^{*}\left(\left(\prod_{i=1}^{k} \nu^{\alpha_{i}} \rho\right) \times \delta\left(\left[\nu^{-(a-1) / 2} \rho, \nu^{(a-1) / 2} \rho\right]\right) \rtimes \pi\right) \\
& \quad=\left(\prod_{i=1}^{k}\left(1 \otimes \nu^{\alpha_{i}} \rho+\nu^{\alpha_{i}} \rho \otimes 1+\nu^{-\alpha_{i}} \rho \otimes 1\right)\right)
\end{aligned}
$$

$$
\begin{gathered}
\times\left(\sum_{i^{\prime}=-(a-1) / 2-1}^{(a-1) / 2} \sum_{j^{\prime}=i^{\prime}}^{(a-1) / 2} \delta\left(\left[\nu^{-i^{\prime}} \rho, \nu^{(a-1) / 2} \rho\right]\right) \times \delta\left(\left[\nu^{j^{\prime}+1} \rho, \nu^{(a-1) / 2} \rho\right]\right)\right. \\
\left.\otimes \delta\left(\left[\nu^{i^{\prime}+1} \rho, \nu^{j^{\prime}} \rho\right]\right)\right) \rtimes \mu^{*}(\pi) .
\end{gathered}
$$

Denote $\Psi=\left(\prod_{i=1}^{k} \nu^{\alpha_{i}} \rho\right) \times \delta\left(\left[\nu^{-(a-1) / 2} \rho, \nu^{(a-1) / 2} \rho\right]\right) \rtimes \pi$.

Let $\tau$ be an irreducible representation of $G L\left(k p, F^{\prime}\right)$ such that $\bigotimes_{i=1}^{k} \nu^{\alpha_{i}} \rho$ is a subquotient of a corresponding Jacquet module of $\tau$. Then the cuspidal support of $\tau$ is $\nu^{\alpha_{1}} \rho, \nu^{\alpha_{2}} \rho, \ldots, \nu^{\alpha_{k}} \rho$. Let $\sigma$ be an irreducible representation of $S_{q-k p}$. Each of the representations $\left(\otimes_{i=1}^{k} \nu^{\alpha_{i}} \rho\right) \otimes T,\left(\bigotimes_{i=1}^{k} \nu^{\alpha_{i}} \rho\right) \otimes \delta\left(\left[\nu^{-(a-1) / 2} \rho, \nu^{(a-1) / 2} \rho\right]\right) \otimes \pi$, which is a subquotient of a corresponding Jacquet module of $\Psi$, is a subquotient of some Jacquet module of $\tau \otimes \sigma$ as above (we use the transitivity of Jacquet modules).

First we shall analyze which terms in the above sum (4-10) - (4-11) can give (after further multiplication) $\tau \otimes \sigma$ as above, for a subquotient. We shall first discuss how one can choose terms in the sum in (4-11). Suppose that for some indexes $i^{\prime}, j^{\prime}$ we have $-i^{\prime} \leq(a-1) / 2$ or $j^{\prime}+1 \leq(a-1) / 2$. Then we would have $\nu^{(a-1) / 2} \rho$ in the cuspidal support of $\tau$, which contradicts (i). Thus we always have $i^{\prime}=-(a-1) / 2-1$ and $j^{\prime}=(a-1) / 2$. Therefore, there is only one possibility for the term in the sum in (4-11). This term is

$$
1 \otimes \delta\left(\left[\nu^{-(a-1) / 2} \rho, \nu^{(a-1) / 2} \rho\right]\right) .
$$

Now we shall discuss how one can choose the terms in the product (4-10) . First of all, since $\alpha_{i}>0$ for all $i$, we must not take $\nu^{-\alpha_{i}} \rho \otimes 1$, since $\nu^{-\alpha_{i}} \rho$ are not in the cuspidal support of $\tau$. Thus, we must take either $1 \otimes \nu^{\alpha_{i}} \rho$ or $\nu^{\alpha_{i}} \rho \otimes 1$.

Suppose that for some index $i$, we have chosen $1 \otimes \nu^{\alpha_{i}} \rho$ (which gives $\tau \otimes \sigma$ for a subquotient after further multiplication). Since $\nu^{\alpha_{i}} \rho$ is in the cuspidal support of $\tau$, and we have seen that in (4-11) we must take the term $1 \otimes \delta\left(\left[\nu^{-(a-1) / 2} \rho, \nu^{(a-1) / 2} \rho\right]\right)$, to be able to get $\tau \otimes \sigma$ for a subquotient, we must have an irreducible representation 
$\tau^{\prime} \otimes \sigma^{\prime}$ such that

$$
\tau^{\prime} \otimes \sigma^{\prime} \leq \mu^{*}(\pi), \operatorname{Supp}\left(\tau^{\prime}\right) \subseteq\left\{\nu^{\alpha_{1}} \rho, \ldots, \nu^{\alpha_{k}} \rho\right\} \text { and } \nu^{\alpha_{i}} \rho \in \operatorname{Supp}\left(\tau^{\prime}\right)
$$

where here Supp is the cuspidal support of a representation of a general linear group, viewed as an unordered collection of cuspidal representations. In fact, (ii) implies that $\operatorname{Supp}(\tau)$ and $\operatorname{Supp}\left(\tau^{\prime}\right)$ are actually the sets. The above discussion and Lemma 3.2 imply that $\nu^{\alpha_{j}} \rho \otimes \sigma^{\prime \prime} \leq \mu^{*}(\pi)$ for some $\nu^{\alpha_{j}} \rho \in \operatorname{Supp}\left(\tau^{\prime}\right)$ and some irreducible representation $\sigma^{\prime \prime}$. Lemma 3.6 implies $\left(\rho, 2 \alpha_{j}+1\right) \in \operatorname{Jord}(\pi)$. This contradicts (iii). Therefore, we must always take terms $\nu^{\alpha_{i}} \rho \otimes 1$ in the product.

Thus, $\tau \otimes \sigma$ must be a subquotient $\left(\prod_{i=1}^{k} \nu^{\alpha_{i}} \rho\right) \otimes \delta\left(\left[\nu^{-(a-1) / 2} \rho, \nu^{(a-1) / 2} \rho\right]\right) \rtimes \pi$. Note that $\prod_{i=1}^{k} \nu^{\alpha_{i}} \rho$ is a regular representation of $G L\left(k p, F^{\prime}\right)$. Since

$$
\delta\left(\left[\nu^{-(a-1) / 2} \rho, \nu^{(a-1) / 2} \rho\right]\right) \rtimes \pi
$$

is a multiplicity one representation by (i) of Lemma 4.3 and Frobenius reciprocity, the multiplicity one of $\left(\bigotimes_{i=1}^{k} \nu^{\alpha_{i}} \rho\right) \otimes T$ claimed in the lemma directly follows. Further, the claim of the lemma about multiplicity two of $\left(\otimes_{i=1}^{k} \nu^{\alpha_{i}} \rho\right) \otimes$ $\delta\left(\left[\nu^{-(a-1) / 2} \rho, \nu^{(a-1) / 2} \rho\right]\right) \otimes \pi$ follows from (i) of Lemma 4.3. The claims about the number of irreducible subrepresentations follow from Frobenius reciprocity and the above multiplicities which we have calculated.

The previous part of the proof implies that if

$$
\left(\prod_{i=1}^{k} \nu^{\alpha_{i}} \rho\right) \times \delta\left(\left[\nu^{-(a-1) / 2} \rho, \nu^{(a-1) / 2} \rho\right]\right) \rtimes \pi
$$

has two irreducible subrepresentations, say $\pi_{1}$ and $\pi_{-1}$, then

$$
\delta\left(\left[\nu^{-(a-1) / 2} \rho, \nu^{(a-1) / 2} \rho\right]\right) \rtimes \pi
$$

reduces to a sum of irreducible representations $T_{1} \oplus T_{-1}$. Further, $\pi_{i} \hookrightarrow\left(\prod_{i=1}^{k} \nu^{\alpha_{i}} \rho\right)$ $\rtimes T_{i}$ for $i= \pm 1$, or $\pi_{i} \hookrightarrow\left(\prod_{i=1}^{k} \nu^{\alpha_{i}} \rho\right) \rtimes T_{-i}$ for $i= \pm 1$. This implies that $\left(\otimes_{i=1}^{k} \nu^{\alpha_{i}} \rho\right) \otimes T_{i}$ is a subquotient of a corresponding Jacquet module of $\pi_{i}$ for $i= \pm 1$, or $\left(\otimes_{i=1}^{k} \nu^{\alpha_{i}} \rho\right) \otimes T_{-i}$ is a subquotient of a corresponding Jacquet module of $\pi_{i}$ for $i= \pm 1$. Now the first part of the proof implies $\pi_{1} ¥ \pi_{-1}$.

Note that for the proof of the above lemma we could suppose a weaker condition instead of (i): $(a-1) / 2 \notin\left\{a_{1}, \alpha_{2}, \ldots, \alpha_{k}\right\}$. Actually, the lemma holds in a much bigger generality, but we prove a version which is adapted for our most frequent applications.

4.6. Lemma. Let $\rho$ be an irreducible cuspidal $F^{\prime} / F$-selfdual representation of $G L\left(p, F^{\prime}\right)$ and let $\pi$ be an irreducible square integrable representation of $S_{q}$. Let $a_{-}, a \in \mathbb{N}, a_{-}<a$. Suppose that $\delta\left(\left[\nu^{-\left(a_{-}-1\right) / 2} \rho, \nu^{\left(a_{-}-1\right) / 2} \rho\right]\right) \rtimes \pi$ reduces. Write

$$
\delta\left(\left[\nu^{-\left(a_{-}-1\right) / 2} \rho, \nu^{\left(a_{-}-1\right) / 2} \rho\right]\right) \rtimes \pi=T_{1} \oplus T_{-1},
$$


where $T_{\eta}$ are irreducible and $T_{1} \neq T_{-1}$ (we can do this by (i) of Lemma 4.3). Suppose that $(\rho, b) \notin \operatorname{Jord}(\pi)$ for $a_{-} \leq b \leq a$. For $\eta \in\{ \pm 1\}$ denote

$$
\begin{aligned}
& \sigma_{\eta}=\left(\begin{array}{cc}
(a-1) / 2-\left(\left(a_{-}-1\right) / 2+1\right) & \bigotimes^{(a-1) / 2-i} \rho \\
\bigotimes_{i=0} &
\end{array} T_{\eta},\right. \\
& \pi_{0}=\delta\left(\left[\nu^{-\left(a_{-}-1\right) / 2} \rho, \nu^{(a-1) / 2} \rho\right]\right) \rtimes \pi, \\
& \pi_{\eta}=\left(\prod_{i=0}^{(a-1) / 2-\left(\left(a_{-}-1\right) / 2+1\right)} \nu^{(a-1) / 2-i} \rho\right) \rtimes T_{\eta}, \\
& \pi_{\eta}^{\prime}=\delta\left(\left[\nu^{\left(a_{-}-1\right) / 2+1} \rho, \nu^{(a-1) / 2} \rho\right]\right) \rtimes T_{\eta} .
\end{aligned}
$$

Then:

(i) The multiplicity of $\sigma_{\eta}$ in corresponding Jacquet modules of $\pi_{0}, \pi_{\eta}$ and $\pi_{\eta}^{\prime}$ is one.

(ii) The multiplicity of $\sigma_{\eta}$ in corresponding Jacquet modules of $\pi_{-\eta}$ and $\pi_{-\eta}^{\prime}$ is zero.

Proof. Let $k \in \mathbb{Z}_{+}$. For an irreducible representation $\gamma$ of $S_{q}$ and $\tau \otimes \sigma$ of $M_{\left(p^{\prime}\right)} \cong$ $G L\left(p^{\prime}, F^{\prime}\right) \times S_{q-p^{\prime}}$ we shall write

$$
\operatorname{Mult}\left(\tau \otimes \sigma, \mu^{*}(\gamma)\right)=k
$$

if $k(\tau \otimes \sigma) \leq \mu^{*}(\gamma)$ and $(k+1)(\tau \otimes \sigma) \not \leq \mu^{*}(\gamma)$.

Denote

$$
\sigma_{\eta}^{\prime}=\delta\left(\left[\nu^{\left(a_{-}-1\right) / 2+1} \rho, \nu^{(a-1) / 2} \rho\right]\right) \otimes T_{\eta} .
$$

Then the multiplicity of $\sigma_{\eta}^{\prime}$ in corresponding Jacquet modules of $\pi_{0}, \pi_{\eta}, \pi_{-\eta}, \pi_{\eta}^{\prime}$ and $\pi_{-\eta}^{\prime}$ is equal to the multiplicity of $\sigma_{\eta}$ in corresponding Jacquet modules of $\pi_{0}, \pi_{\eta}$, $\pi_{-\eta}, \pi_{\eta}^{\prime}$ and $\pi_{-\eta}^{\prime}$, respectively (this follows from the well-known characterization of $\delta\left(\left[\nu^{\left(a_{-}-1\right) / 2+1} \rho, \nu^{(a-1) / 2} \rho\right]\right)$ in terms of Jacquet modules).

Using (1-1) and (1-3) we get

$$
\begin{aligned}
& \mu^{*}\left(\delta\left(\left[\nu^{-\left(a_{-}-1\right) / 2} \rho, \nu^{(a-1) / 2} \rho\right]\right) \rtimes \pi\right) \\
& =\left(\sum_{i=-\left(a_{-}-1\right) / 2-1}^{(a-1) / 2} \sum_{j=i}^{(a-1) / 2} \delta\left(\left[\nu^{-i} \rho, \nu^{\left(a_{-}-1\right) / 2} \rho\right]\right) \times \delta\left(\left[\nu^{j+1} \rho, \nu^{(a-1) / 2} \rho\right]\right)\right. \\
& \left.\otimes \delta\left(\left[\nu^{i+1} \rho, \nu^{j} \rho\right]\right)\right) \rtimes \mu^{*}(\pi) .
\end{aligned}
$$

Note that

$$
\pi_{\eta}^{\prime} \leq \pi_{\eta}
$$

Since

$$
\begin{aligned}
& \delta\left(\left[\nu^{\left(a_{-}-1\right) / 2+1} \rho, \nu^{(a-1) / 2} \rho\right]\right) \otimes \delta\left(\left[\nu^{-\left(a_{-}-1\right) / 2} \rho, \nu^{\left(a_{-}-1\right) / 2} \rho\right]\right) \\
& \leq M^{*}\left(\delta\left(\left[\nu^{-\left(a_{-}-1\right) / 2} \rho, \nu^{(a-1) / 2} \rho\right]\right)\right)
\end{aligned}
$$

(take in (4-13) indexes $\left.i=-\left(a_{-}-1\right) / 2-1, j=\left(a_{-}-1\right) / 2\right)$, we get

$$
1 \leq \operatorname{Mult}\left(\sigma_{\eta}^{\prime}: \mu^{*}\left(\pi_{0}\right)\right) \text {. }
$$


Frobenius reciprocity implies

$$
1 \leq \operatorname{Mult}\left(\sigma_{\eta}^{\prime}: \mu^{*}\left(\pi_{\eta}^{\prime}\right)\right) .
$$

Now (4-14) implies

$$
1 \leq \operatorname{Mult}\left(\sigma_{\eta}^{\prime}: \mu^{*}\left(\pi_{\eta}\right)\right)
$$

Note that

$$
\pi_{0} \leq \pi_{1}^{\prime}+\pi_{-1}^{\prime} \leq \pi_{1}+\pi_{-1}
$$

Lemma 4.5 implies

$$
\operatorname{Mult}\left(\sigma_{\eta}, \mu^{*}\left(\pi_{1}+\pi_{-1}\right)\right)=\operatorname{Mult}\left(\sigma_{\eta}, \mu^{*}\left(\pi_{\eta}\right)\right)=1 .
$$

Now from (4-14) - (4-19) we can conclude all the claims of the lemma.

The next corollary follows directly from the above lemma.

4.7. Corollary ( $\mathrm{M} 2$, Remark 5.1.1). Let the notation and the assumptions be the same as in the above lemma. Then

(i) The representation $\pi_{0}$ has exactly two irreducible subrepresentations. They are not isomorphic.

(ii) The representation $\pi_{\eta}$ has a unique irreducible subrepresentation. This subrepresentation is a unique irreducible subrepresentation of $\pi_{\eta}^{\prime}$. The corresponding two irreducible subrepresentations, for $\eta= \pm 1$, are irreducible subrepresentations of $\pi_{0}$.

(iii) The representations $\pi_{\eta}^{\prime}$ are reducible.

Proof. We get (i) and (ii) directly from $\pi_{0} \hookrightarrow \pi_{1} \oplus \pi_{-1}$ and the multiplicities in the above lemma. Namely, $\pi_{1}$ and $\pi_{-1}$ have unique irreducible subrepresentations and they are not isomorphic (this follows from the above lemma). Further, these irreducible subrepresentations must show up in $\pi_{0}$ because of the multiplicities calculated in the above lemma. It remains to prove (iii).

Suppose $\pi_{\eta}^{\prime}$ is irreducible. Then $\pi_{\eta}^{\prime} \leq \pi_{0}$ by (ii). This implies $\mu^{*}\left(\pi_{\eta}^{\prime}\right) \leq \mu^{*}\left(\pi_{0}\right)$. Therefore, $\delta\left(\left[\nu^{-(a-1) / 2} \rho, \nu^{-\left(a_{-}-1\right) / 2-1} \rho\right]\right) \otimes T_{\eta}$ is a subquotient of (4-13) (see Lemma 4.4). Since $a, a_{-}>0$, to be able to get $\delta\left(\left[\nu^{-(a-1) / 2} \rho, \nu^{-\left(a_{-}-1\right) / 2-1} \rho\right]\right) \otimes T_{\eta}$ for a subquotient of a term in the sum (4-13) we need to have $-i>\left(a_{-}-1\right) / 2$ and $j+1>(a-1) / 2$. This implies $i=-\left(a_{-}-1\right) / 2-1$ and $j=(a-1) / 2$. Thus, $\delta\left(\left[\nu^{-(a-1) / 2} \rho, \nu^{-\left(a_{-}-1\right) / 2-1} \rho\right]\right) \otimes T_{\eta}$ must be a subquotient of

$$
1 \otimes \delta\left(\left[\nu^{-\left(a_{-}-1\right) / 2} \rho, \nu^{(a-1) / 2} \rho\right]\right) \rtimes \mu^{*}(\pi) .
$$

Therefore, we must have $\delta\left(\left[\nu^{-(a-1) / 2} \rho, \nu^{-\left(a_{-}-1\right) / 2-1} \rho\right]\right) \otimes \tau \leq \mu^{*}(\pi)$ for some irreducible $\tau$. This contradicts the square integrability of $\pi$. The proof is now complete.

\section{Factors of Square integrable Representations And Jord}

We shall very often use the following lemma, which is already in 5.1 of [M2].

5.1. Lemma. Let $\pi$ be an irreducible square integrable representation of $S_{q}$ and let $\rho$ be an irreducible $F^{\prime} / F$-selfdual cuspidal representation of $G L\left(p, F^{\prime}\right)$. Suppose that we have $a \in \operatorname{Jord}_{\rho}(\pi)$ such that $a_{-}$is defined and that the following holds:

$$
\epsilon_{\pi}(\rho, a)=\epsilon_{\pi}\left(\rho, a_{-}\right) \text {. }
$$


Denote $\operatorname{Jord}^{\prime}=\operatorname{Jord}(\pi) \backslash\left\{(\rho, a),\left(\rho, a_{-}\right)\right\}$. Let $\operatorname{Jord}^{\prime}, \pi_{\text {cusp }}, \epsilon^{\prime}$ be the subordinated triple to $\operatorname{Jord}(\pi), \pi_{c u s p}, \epsilon_{\pi}$. Then there exists an irreducible square integrable representation $\pi^{\prime}$ of $S_{q-p\left(a+a_{-}\right) / 2}$ such that $\operatorname{Jord}\left(\pi^{\prime}\right)=J$ ord $d^{\prime}, \pi_{c u s p}^{\prime}=\pi_{\text {cusp }}$ and $\epsilon_{\pi^{\prime}}=\epsilon^{\prime}$. Further

$$
\pi \hookrightarrow \delta\left(\left[\nu^{-\left(a_{-}-1\right) / 2} \rho, \nu^{(a-1) / 2} \rho\right]\right) \rtimes \pi^{\prime} .
$$

Proof. Since $\epsilon_{\pi}(\rho, a)=\epsilon_{\pi}\left(\rho, a_{-}\right)$, we have $\pi \hookrightarrow \delta\left(\left[\nu^{\left(a_{-}-1\right) / 2+1} \rho, \nu^{(a-1) / 2} \rho\right]\right) \rtimes \tau$ for some irreducible $\tau$. The first lemma in the fifth section of [M2] implies

$$
\tau \hookrightarrow \delta\left(\left[\nu^{-\left(a_{-}-1\right) / 2} \rho, \nu^{\left(a_{-}-1\right) / 2} \rho\right]\right) \rtimes \pi^{\prime}
$$

for the irreducible square integrable representation $\pi^{\prime}$ satisfying

$$
\left(\operatorname{Jord}\left(\pi^{\prime}\right),\left(\pi^{\prime}\right)_{\text {cusp }}, \epsilon_{\pi^{\prime}}\right)=\left(\operatorname{Jord}^{\prime}, \pi_{\text {cusp }}, \epsilon^{\prime}\right) .
$$

Thus

$$
\pi \hookrightarrow \delta\left(\left[\nu^{\left(a_{-}-1\right) / 2+1} \rho, \nu^{(a-1) / 2} \rho\right]\right) \times \delta\left(\left[\nu^{-\left(a_{-}-1\right) / 2} \rho, \nu^{\left(a_{-}-1\right) / 2} \rho\right]\right) \rtimes \pi^{\prime} .
$$

Note that

$$
\begin{aligned}
& \delta\left(\left[\nu^{-\left(a_{-}-1\right) / 2} \rho, \nu^{(a-1) / 2} \rho\right]\right) \rtimes \pi^{\prime} \\
& \quad \hookrightarrow \delta\left(\left[\nu^{\left(a_{-}-1\right) / 2+1} \rho, \nu^{(a-1) / 2} \rho\right]\right) \times \delta\left(\left[\nu^{-\left(a_{-}-1\right) / 2} \rho, \nu^{\left(a_{-}-1\right) / 2} \rho\right]\right) \rtimes \pi^{\prime} .
\end{aligned}
$$

By Lemma 4.5 (and Remark 4.2), the representation

$$
\delta\left(\left[\nu^{\left(a_{-}-1\right) / 2+1} \rho, \nu^{(a-1) / 2} \rho\right]\right) \times \delta\left(\left[\nu^{-\left(a_{-}-1\right) / 2} \rho, \nu^{\left(a_{-}-1\right) / 2} \rho\right]\right) \rtimes \pi^{\prime}
$$

has exactly two irreducible subrepresentations. Since Corollary 4.7 implies that the representation $\delta\left(\left[\nu^{-\left(a_{-}-1\right) / 2} \rho, \nu^{(a-1) / 2} \rho\right]\right) \rtimes \pi^{\prime}$ also has exactly two irreducible subrepresentations, (5-2) and (5-3) imply (5-1).

Let $\pi$ be an irreducible square integrable representation of $S_{q}$. If $\pi$ is strongly positive, then by definition

$$
e(\tau)>0
$$

for each factor $\tau$ of $\pi$. Further, the admissible triple $\operatorname{Jord}(\pi), \pi_{\text {cusp }}, \epsilon_{\pi}$ is of alternated type ([M2], 5.3). Suppose that $\pi$ is not strongly positive. Then $\operatorname{Jord}(\pi), \pi_{\text {cusp }}$, $\epsilon_{\pi}$ is of mixed type. The following lemma gives us general information about factors of square integrable representations which are not strongly positive.

To simplify discussion in some cases, we shall often in the sequel restrict to the case where the admissible triple $J o r d, \pi_{c u s p}, \epsilon$ satisfies the following condition: there exists an irreducible cuspidal $F^{\prime} / F$-selfdual representation $\rho$ of $G L\left(p, F^{\prime}\right)$ such that

$$
\operatorname{Jord} \backslash\left\{(\rho, a) ; a \in \operatorname{Jord}_{\rho}\right\} \subseteq \operatorname{Jord}\left(\pi_{\text {cusp }}\right) .
$$

This means that we are looking only at the irreducible square integrable representations which are subquotients of the parabolically induced representations of the following type:

$$
\left(\prod_{i=1}^{\ell} \nu^{x_{i}} \rho\right) \rtimes \pi_{c u s p}
$$

where $\ell$ is a non-negative integer and $x_{i} \in \mathbb{R}, i=1, \ldots, \ell$.

We shall say that an irreducible square integrable representation $\pi$ of $S_{q}$ satisfies (L) if $\operatorname{Jord}(\pi), \pi_{\text {cusp }}, \epsilon_{\pi}$ satisfies (L). The representation $\rho$ in condition (L) will always be fixed in such a way that it is clear which $\rho$ is being considered. 
5.2. Lemma. Suppose that $\pi$ is an irreducible square integrable representation of $S_{q}$ which is not strongly positive. Take any $(\rho, a) \in \operatorname{Jord}(\pi)$ such that $a_{-} \in$ $\operatorname{Jord}_{\rho}(\pi)$ is defined, and that $\epsilon_{\pi}(\rho, a)=\epsilon_{\pi}\left(\rho, a_{-}\right)$. Suppose that $(L)$ holds. Define $a_{\rho}(\pi) \in \operatorname{Jord}_{\rho}(\pi)$ to be maximal with these properties (then $a_{\rho}(\pi)_{-} \in \operatorname{Jord}_{\rho}(\pi)$ is defined and $\left.\epsilon_{\pi}\left(\rho, a_{\rho}(\pi)\right)=\epsilon_{\pi}\left(\rho, a_{\rho}(\pi)_{-}\right)\right)$. Now if $\tau=\nu^{k} \rho, k \in \mathbb{R}$, is a factor of $\pi$, then

$$
k=e(\tau) \geq-\left(a_{\rho}(\pi)_{-}-1\right) / 2 .
$$

Proof. The proof proceeds by induction with respect to $q$. Denote

$$
a=a_{\rho}(\pi) .
$$

By the above lemma, there exists an irreducible square integrable representation $\pi^{\prime}$ such that

$$
\pi \hookrightarrow \delta\left(\left[\nu^{-\left(a_{-}-1\right) / 2} \rho, \nu^{(a-1) / 2} \rho\right]\right) \rtimes \pi^{\prime},
$$

where

$$
\operatorname{Jord}_{\rho}\left(\pi^{\prime}\right)=\operatorname{Jord}_{\rho}(\pi) \backslash\left\{a, a_{-}\right\}
$$

and $\operatorname{Jord}\left(\pi^{\prime}\right), \pi_{c u s p}^{\prime}, \epsilon_{\pi^{\prime}}$ is subordinated to $\operatorname{Jord}(\pi), \pi_{c u s p}, \epsilon_{\pi}$. Note that (5-6) and the inductive assumption imply

either $\pi^{\prime}$ is strongly positive, or $a_{\rho}\left(\pi^{\prime}\right)$ is defined and $a_{\rho}\left(\pi^{\prime}\right)_{-}<a_{-}=a_{\rho}(\pi)_{-}$.

Thus, the inductive assumption or the fact that $\pi^{\prime}$ is strongly positive if $\pi^{\prime}$ is actually strongly positive implies that $\nu^{\alpha} \rho$ is not a factor of $\pi^{\prime}$ if $\alpha \leq-\left(a_{-}-1\right) / 2$.

Suppose that (5-4) does not hold. Let $\tau=\nu^{k} \rho$ be a factor for which (5-4) does not hold. Fix such a factor $\tau=\nu^{k} \rho$ with minimal possible $k$. Note that $(5-5)$, the fact that $\nu^{\alpha} \rho$ is not a factor of $\pi^{\prime}$ if $\alpha \leq-\left(a_{-}-1\right) / 2,(1-2)$ and (1-4) imply that

$$
k \in\left[-(a-1) / 2,-\left(a_{-}-1\right) / 2-1\right]
$$

(and $k+(a-1) / 2 \in \mathbb{Z})$. Clearly $k \leq-1$. By the choice of $k, \tau=\nu^{k} \rho$ satisfies the assumption of Lemma 3.4 (and also (5-8)). Let $c=-2 k+1$ (then $k=-(c-1) / 2$ and $c \geq 3)$. Now (5-8) implies $-(a-1) / 2 \leq-(c-1) / 2<-\left(a_{-}-1\right) / 2$. Thus

$$
a_{-}<c \leq a \text {. }
$$

Lemma 3.4 implies

$$
\pi \hookrightarrow \delta\left(\left[\nu^{-(c-1) / 2} \rho, \nu^{(b-1) / 2} \rho\right]\right) \rtimes \pi^{\prime \prime}
$$

for some $b \in c+2 \mathbb{Z}$ which satisfies $-(c-1) / 2 \leq(b-1) / 2$ (i.e. $b+c-2 \geq 0)$. The square integrability of $\pi$ and (5-10) (and Frobenius reciprocity) imply

$$
c<b \text {. }
$$

Since (by our choice) $k$ is minimal such that $\nu^{k} \rho$ is a factor of $\pi$, there is no embedding $\pi \hookrightarrow \delta\left(\left[\nu^{-\left(c^{\prime}-1\right) / 2} \rho, \nu^{(b-1) / 2} \rho\right]\right) \rtimes \pi^{\prime \prime \prime}$, for some irreducible $\pi^{\prime \prime \prime}$ with $-\left(c^{\prime}-1\right) / 2<(c-1) / 2$. Therefore, Remark 3.2 of [M2 implies that $\pi^{\prime \prime}$ is square integrable. Proposition 2.1 now implies

$$
b, c \in \operatorname{Jord}_{\rho}(\pi) \text {. }
$$

From (5-9), (5-11) and (5-12) we get

$$
a \leq c<b .
$$


This implies that $b_{-} \in \operatorname{Jord}_{\rho}(\pi)$ is defined and (5-13) implies $b_{-} \geq c$. Now (5-10), Lemma 3.2 and the definition of $\epsilon_{\pi}$ imply

$$
\epsilon_{\pi}(\rho, b)=\epsilon_{\pi}\left(\rho, b_{-}\right)
$$

(see also Lemma 5.2 in [M2]).

Note that (5-14) implies $b \leq a=a_{\rho}(\pi)$. This contradicts (5-13). This contradiction completes the proof.

We shall include here the following lemma, although we shall not use it in this paper. This lemma complements Lemma 5.4.1 of [M2].

5.3. Lemma. Let $\pi$ be an irreducible square integrable representation. Suppose $a \in \operatorname{Jord}_{\rho}(\pi)$ and $a+2 \notin \operatorname{Jord}_{\rho}(\pi)$. Then

$$
\nu^{(a+1) / 2} \rho \rtimes \pi
$$

reduces. Further, it contains a unique irreducible subrepresentation.

Proof. Suppose that $\nu^{(a+1) / 2} \rho \rtimes \pi$ is irreducible. Let $\tau$ be an irreducible subrepresentation of $\delta\left(\left[\nu^{-(a+1) / 2} \rho, \nu^{(a+1) / 2} \rho\right]\right) \rtimes \pi$. Then

$$
\begin{aligned}
& \tau \hookrightarrow \delta\left(\left[\nu^{-(a+1) / 2} \rho, \nu^{(a+1) / 2} \rho\right]\right) \rtimes \pi \\
& \hookrightarrow \delta\left(\left[\nu^{-(a+1) / 2+1} \rho+1, \nu^{(a+1) / 2} \rho\right]\right) \times \nu^{-(a+1) / 2} \rho \rtimes \pi \\
& \quad \cong \delta\left(\left[\nu^{-(a+1) / 2+1} \rho+1, \nu^{(a+1) / 2} \rho\right]\right) \times \nu^{(a+1) / 2} \rho \rtimes \pi \\
& \cong \nu^{(a+1) / 2} \rho \times \delta\left(\left[\nu^{-(a+1) / 2+1} \rho+1, \nu^{(a+1) / 2} \rho\right]\right) \rtimes \pi \\
& \hookrightarrow \nu^{(a+1) / 2} \rho \times \nu^{(a+1) / 2} \rho \times \delta\left(\left[\nu^{-(a+1) / 2+1} \rho+1, \nu^{(a+1) / 2-1} \rho\right]\right) \rtimes \pi .
\end{aligned}
$$

Now Frobenius reciprocity implies that

$$
\tau^{\prime}=\nu^{(a+1) / 2} \rho \times \nu^{(a+1) / 2} \rho \otimes \delta\left(\left[\nu^{-(a-1) / 2} \rho+1, \nu^{(a-1) / 2} \rho\right]\right) \rtimes \pi
$$

is a subquotient of a corresponding Jacquet module of $\pi$ (note that $\tau^{\prime}$ is irreducible).

Now we get directly that the multiplicity of $\tau^{\prime}$ in $\mu^{*}\left(\delta\left(\left[\nu^{-(a+1) / 2} \rho, \nu^{(a+1) / 2} \rho\right]\right) \rtimes \pi\right)$ is one (use $\left.a+2 \notin \operatorname{Jord}_{\rho}(\pi)\right)$. This proves irreducibility of $\delta\left(\left[\nu^{-(a+1) / 2} \rho, \nu^{(a+1) / 2} \rho\right]\right)$ $\rtimes \pi$, which contradicts our assumption $a+2 \notin \operatorname{Jord}_{\rho}(\pi)\left(\right.$ note that $a \in \operatorname{Jord}_{\rho}(\pi)$ and therefore $a$ and also $a+2$ satisfy (J-1)). Thus, we have proved reducibility. The uniqueness of the irreducible subrepresentation follows from Lemma 4.5.

Having in mind Lemma 5.4.1 of [M2] and the above (simple) lemma, it remains to describe a criterion for reducibility of $\nu^{(a+1) / 2} \rho \rtimes \pi$ when $a \in \operatorname{Jord}_{\rho}(\pi)$ and $a+2 \in \operatorname{Jord}_{\rho}(\pi)$. The criterion is the following: we have irreducibility if and only if

$$
\epsilon_{\pi}(\rho, a+2) \neq \epsilon_{\pi}(\rho, a) .
$$

This is proved using intertwining operators. The composition of the two standard intertwining operators

$$
\nu^{(a+1) / 2+s} \rho \rtimes \pi \rightarrow \nu^{-(a+1) / 2-s} \rho \rtimes \pi \rightarrow \nu^{(a+1) / 2+s} \rho \rtimes \pi
$$

is holomorphic and non-zero at $s=0$ under the hypothesis that $(\rho, a)$ and $(\rho, a+2)$ are in $\operatorname{Jord}(\pi)$. Moreover the first one is holomorphic at $s=0$ by a general result of Harish-Chandra. Therefore, we need to prove that the second one is also holomorphic. This can be done by an inductive argument with respect to $\pi$ (since 
the argument is long, and we shall not use this result in this paper, we shall not write it down here).

\section{THEOREM}

If we consider the series of odd-orthogonal (resp. symplectic) groups, then the degree $\operatorname{deg}($ Jord $)$ of an admissible triple Jord, $\pi_{\text {cusp }}, \epsilon$ is defined to be $n$ if $\sum_{(\rho, a) \in J \text { Jord }} a d_{\rho}=2 n\left(\operatorname{resp} . \sum_{(\rho, a) \in J o r d} a d_{\rho}=2 n+1\right)$. In [M2], it is proved that the mapping

$$
\pi \rightarrow\left(\operatorname{Jord}(\pi), \pi_{\text {cusp }}, \epsilon_{\pi}\right)
$$

is an injective mapping from the set of all equivalence classes of irreducible square integrable representations of $S_{n}$ into the set of all admissible triples of degree $n$. In this paper we shall prove that this mapping is surjective. In this way we shall prove the following

6.1. Theorem. Assume that (BA) holds. Then the map

$$
\pi \rightarrow\left(\operatorname{Jord}(\pi), \pi_{\text {cusp }}, \epsilon_{\pi}\right)
$$

defines a bijection from the set of all equivalence classes of irreducible square integrable representations of groups $S_{n}$ onto the set of all admissible triples.

Suppose further that besides (BA), the equality in (A) also holds. Under these assumptions, the map $\pi \rightarrow\left(\operatorname{Jord}(\pi), \pi_{\text {cusp }}, \epsilon_{\pi}\right)$ is a bijection from the set of all equivalence classes of irreducible square integrable representations of the group $S_{n}$ onto the set of all admissible triples of degree $n$.

In the next five sections we shall prove this theorem.

\section{Strongly POSitive IRREDUCible REPRESENTATiOns}

Let Jord, $\pi_{c u s p}, \epsilon$ be an admissible triple of alternated type. In the first part of this section we shall assume that (L) holds for this triple. When we work with Jord which satisfies (L), then for $a \in \operatorname{Jord}_{\rho}$ we will usually denote $\epsilon(\rho, a)$ simply by $\epsilon(a)$. Also the function $\phi_{\rho}$ will be denoted simply by $\phi$. Note that if $\rho^{\prime} \neq \rho$, then $\phi_{\rho^{\prime}}(b)=b$ for $b \in \operatorname{Jord}_{\rho^{\prime}}=\operatorname{Jord}_{\rho^{\prime}}\left(\pi_{\text {cusp }}\right)$.

Write the elements of $\operatorname{Jord}_{\rho}$ as

$$
a_{1}<a_{2}<\cdots<a_{k}
$$

(the possibility $k=0$ is not excluded). Recall that $\operatorname{Jord}_{\rho} \subseteq 2 \mathbb{N}$ or $1+2 \mathbb{Z}_{+}$. We shall say that we are in the even case if $\operatorname{Jord}_{\rho} \subseteq 2 \mathbb{N}$, and in the odd case if $\operatorname{Jord}_{\rho} \subseteq 1+2 \mathbb{Z}_{+}$.

If we are in the odd case, then from (2-3) and (2-6) we get

$$
\begin{gathered}
\operatorname{Jord}_{\rho}^{\prime}\left(\pi_{\text {cusp }}\right)=\{1,3, \ldots, 2 k-1\}, \\
\phi:\left\{a_{1}, a_{2}, \ldots, a_{k}\right\} \rightarrow\{1,3, \ldots, 2 k-1\}, \quad a_{i} \mapsto 2 i-1 .
\end{gathered}
$$

In the even case we have two possibilities.

Suppose $\epsilon\left(a_{1}\right)=-1$. Then

$$
\begin{gathered}
\operatorname{Jord}_{\rho}^{\prime}\left(\pi_{\text {cusp }}\right)=\{2,4, \ldots, 2 k\}, \\
\phi:\left\{a_{1}, a_{2}, \ldots, a_{k}\right\} \rightarrow\{2,4, \ldots, 2 k\}, \quad a_{i} \mapsto 2 i .
\end{gathered}
$$


If $\epsilon\left(a_{1}\right)=1$, then

$$
\begin{gathered}
\operatorname{Jord}_{\rho}^{\prime}\left(\pi_{\text {cusp }}\right)=\{0,2,4, \ldots, 2(k-1)\}, \\
\phi:\left\{a_{1}, a_{2}, \ldots, a_{k}\right\} \rightarrow\{0,2,4, \ldots, 2(k-1)\}, \quad a_{i} \mapsto 2(i-1) .
\end{gathered}
$$

Suppose that we have some Jord and $\pi_{\text {cusp }}$ (we do not assume that they form an admissible triple for the moment). Assume that they satisfy (L). Write $\operatorname{Jord}_{\rho}$ as in $(7-1)$.

Let $\operatorname{Jord}_{\rho} \subseteq 1+2 \mathbb{Z}$. Suppose we have a function $\phi$ as in (7-3). Then it is easy to see that there is a unique partially defined function $\epsilon$ such that Jord, $\pi_{\text {cusp }}, \epsilon$ form an admissible triple of alternated type, and that the corresponding function $\phi_{\rho}$ is $\phi$.

Suppose $\operatorname{Jord}_{\rho} \subseteq 2 \mathbb{Z}$ and suppose that a function $\phi$ as in (7-5) (resp. (7-7)) is given. Then again there exists a unique partially defined function $\epsilon$ such that Jord, $\pi_{\text {cusp }}, \epsilon$ form an admissible triple of alternated type such that the corresponding function $\phi_{\rho}$ is $\phi$. One needs to take $\epsilon\left(a_{1}\right)=-1$ (resp. $\epsilon\left(a_{1}\right)=1$ ).

Therefore, we do not need to work with $\epsilon$ 's. Instead we can work directly with functions $\phi$.

Note that (7-1) - (7-7) imply

$$
\begin{gathered}
a_{i} \geq \phi\left(a_{i}\right), \\
a_{i^{\prime}}>\phi\left(a_{i^{\prime}}\right) \quad \Rightarrow \quad a_{i} \geq \phi\left(a_{i}\right)+2 \quad \text { for all } i \geq i^{\prime} .
\end{gathered}
$$

Lemma 4.1 implies that the representation

$$
\sigma_{0}=\left(\prod_{i=1}^{k} \delta\left(\left[\nu^{\left(\phi\left(a_{i}\right)+1\right) / 2} \rho, \nu^{\left(a_{i}-1\right) / 2} \rho\right]\right)\right) \rtimes \pi_{c u s p}
$$

has a unique irreducible subrepresentation. We shall denote this subrepresentation by

$$
\pi=\pi_{\left(J o r d, \pi_{c u s p}, \epsilon\right)}=\pi_{\left(J o r d, \pi_{c u s p}, \phi\right)} .
$$

In this section we shall prove

7.1. Proposition. The representation $\pi$ is strongly positive (square integrable representation).

If $\phi\left(a_{i}\right)=a_{i}$ for all $i$, then $\pi=\pi_{\text {cusp }}$ and the proposition holds. If this is not the case, then $a_{k} \geq \phi\left(a_{k}\right)+2$. Therefore, we shall assume

$$
a_{k} \geq \phi\left(a_{k}\right)+2 .
$$

We shall prove the proposition by induction with respect to the degree of Jord.

Let

$$
j=\min \left\{i ; 1 \leq i \leq k \text { and } a_{i}>\phi\left(a_{i}\right)\right\} .
$$

In the proof of the proposition we shall need the following

7.2. Lemma. (i) The multiplicity of

$$
\begin{aligned}
& \nu^{\left(a_{j}-1\right) / 2} \rho \otimes \delta\left(\left[\nu^{\left(\phi\left(a_{j}\right)+1\right) / 2} \rho, \nu^{\left(a_{j}-1\right) / 2-1} \rho\right]\right) \\
& \otimes\left(\bigotimes_{i=j+1}^{k} \delta\left(\left[\nu^{\left(\phi\left(a_{i}\right)+1\right) / 2} \rho, \nu^{\left(a_{i}-1\right) / 2} \rho\right]\right)\right) \otimes \pi_{c u s p}
\end{aligned}
$$


in the standard Jacquet module of

$$
\begin{aligned}
\sigma_{1}=\nu^{\left(a_{j}-1\right) / 2} \rho \times \delta\left(\left[\nu^{\left(\phi\left(a_{j}\right)+1\right) / 2} \rho, \nu^{\left(a_{j}-1\right) / 2-1} \rho\right]\right) \\
\times\left(\prod_{i=j+1}^{k} \delta\left(\left[\nu^{\left(\phi\left(a_{i}\right)+1\right) / 2} \rho, \nu^{\left(a_{i}-1\right) / 2} \rho\right]\right)\right) \rtimes \pi_{c u s p}
\end{aligned}
$$

is one.

(ii) Suppose $a_{j} \geq \phi\left(a_{j}\right)+4$. Then the multiplicity of

$$
\begin{aligned}
\delta\left(\left[\nu^{\left(a_{j}-1\right) / 2-1} \rho, \nu^{\left(a_{j}-1\right) / 2} \rho\right]\right) \otimes \delta\left(\left[\nu^{\left(\phi\left(a_{j}\right)+1\right) / 2} \rho, \nu^{\left(a_{j}-2\right) / 2-2} \rho\right]\right) \\
\otimes\left(\bigotimes_{i=j+1}^{k} \delta\left(\left[\nu^{\left(\phi\left(a_{i}\right)+1\right) / 2} \rho, \nu^{\left(a_{i}-1\right) / 2} \rho\right]\right)\right) \otimes \pi_{c u s p}
\end{aligned}
$$

in the standard Jacquet module of

$$
\begin{aligned}
\sigma_{2}=\delta\left(\left[\nu^{\left(a_{j}-1\right) / 2-1} \rho, \nu^{\left(a_{j}-1\right) / 2} \rho\right]\right) \times \delta\left(\left[\nu^{\left(\phi\left(a_{j}\right)+1\right) / 2} \rho, \nu^{\left(a_{j}-1\right) / 2-2} \rho\right]\right) \\
\times\left(\prod_{i=j+1}^{k} \delta\left(\left[\nu^{\left(\phi\left(a_{i}\right)+1\right) / 2} \rho, \nu^{\left(a_{i}-1\right) / 2} \rho\right]\right)\right) \rtimes \pi_{c u s p}
\end{aligned}
$$

is one.

(iii) Suppose $a_{j}=\phi\left(a_{j}\right)+2$ and $j<n$. Then the multiplicity of

$$
\begin{aligned}
\nu^{\left(a_{j}-1\right) / 2} \rho \otimes \nu^{\left(a_{j+1}-1\right) / 2} \rho \otimes \delta\left(\left[\nu^{\left(\phi\left(a_{j+1}\right)+1\right) / 2} \rho, \nu^{\left(a_{j+1}-1\right) / 2-1} \rho\right]\right) \\
\otimes\left(\bigotimes_{i=j+2}^{k} \delta\left(\left[\nu^{\left(\phi\left(a_{i}\right)+1\right) / 2} \rho, \nu^{\left(a_{i}-1\right) / 2} \rho\right]\right)\right) \otimes \pi_{c u s p}
\end{aligned}
$$

in the standard Jacquet module of

$$
\begin{aligned}
\sigma_{3}=\nu^{\left(a_{j}-1\right) / 2} \rho \times \nu^{\left(a_{j+1}-1\right) / 2} \rho \times \delta\left(\left[\nu^{\left(\phi\left(a_{j+1}\right)+1\right) / 2} \rho, \nu^{\left(a_{j+1}-1\right) / 2-1} \rho\right]\right) \\
\times\left(\prod_{i=j+2}^{k} \delta\left(\left[\nu^{\left(\phi\left(a_{i}\right)+1\right) / 2} \rho, \nu^{\left(a_{i}-1\right) / 2} \rho\right]\right)\right) \rtimes \pi_{c u s p}
\end{aligned}
$$

is one.

Proof. (i) In the same way as in Lemma 4.1, the proof of (i) reduces to the fact that the multiplicity of $\nu^{\left(a_{j}-1\right) / 2} \rho \otimes \delta\left(\left[\nu^{\left(\phi\left(a_{j}\right)+1\right) / 2} \rho, \nu^{\left(a_{j}-2\right) / 2-1} \rho\right]\right)$ in a corresponding Jacquet module of $\nu^{\left(a_{j}-1\right) / 2} \rho \times \delta\left(\left[\nu^{\left(\phi\left(a_{j}\right)+1\right) / 2} \rho, \nu^{\left(a_{j}-1\right) / 2-1} \rho\right]\right)$ is one. This is true, since we are in the regular situation.

(ii) Again, as in the proof of Lemma 4.1, the proof of (ii) reduces to the fact that the multiplicity of $\delta\left(\left[\nu^{\left(a_{j}-1\right) / 2-1} \rho, \nu^{\left(a_{j}-1\right) / 2} \rho\right]\right) \otimes \delta\left(\left[\nu^{\left(\phi\left(a_{j}\right)+1\right) / 2} \rho, \nu^{\left(a_{j}-2\right) / 2-2} \rho\right]\right)$ in a corresponding Jacquet module of

$$
\delta\left(\left[\nu^{\left(a_{j}-1\right) / 2-1} \rho, \nu^{\left(a_{j}-1\right) / 2} \rho\right]\right) \times \delta\left(\left[\nu^{\left(\phi\left(a_{j}\right)+1\right) / 2} \rho, \nu^{\left(a_{j}-1\right) / 2-2} \rho\right]\right)
$$

is one. This again follows from the regularity.

(iii) The proof of (iii) proceeds in a similar way. The proof reduces to the fact that the multiplicity of $\nu^{\left(a_{j}-1\right) / 2} \rho \otimes \nu^{\left(a_{j+1}-1\right) / 2} \rho \otimes \delta\left(\left[\nu^{\left(\phi\left(a_{j+1}\right)+1\right) / 2} \rho, \nu^{\left(a_{j+1}-1\right) / 2-1} \rho\right]\right)$ in 
a corresponding Jacquet module of

$$
\nu^{\left(a_{j}-1\right) / 2} \rho \times \nu^{\left(a_{j+1}-1\right) / 2} \rho \times \delta\left(\left[\nu^{\left(\phi\left(a_{j+1}\right)+1\right) / 2} \rho, \nu^{\left(a_{j+1}-1\right) / 2-1} \rho\right]\right)
$$

is one.

Note that $a_{j}=\phi\left(a_{j}\right)+2<\phi\left(a_{j+1}\right)+2$. This implies $\left(a_{j}-1\right) / 2<\left(\phi\left(a_{j+1}\right)+1\right) / 2$. Thus, we are again in the regular situation. Therefore, the above multiplicity one holds and further, the multiplicity one claimed in (iii) holds.

Proof of Proposition 7.1. We use the notation from the beginning of this section and after Proposition 7.1. Recall that $\sigma_{0}$ is defined in (7-10).

First we shall define a new triple with the same $\pi_{\text {cusp }}$.

Denote $\operatorname{Jord}^{\prime}=\left(\operatorname{Jord} \backslash\left\{\left(\rho, a_{j}\right)\right\}\right) \cup\left\{\left(\rho, a_{j}-2\right)\right\}$ if $a_{j} \neq 2$. Let $\phi^{\prime}$ be the function on $\operatorname{Jord}_{\rho}^{\prime}$ defined by $\phi^{\prime}\left(a_{j}-2\right)=\phi\left(a_{j}\right)$ and $\phi^{\prime}\left(a_{i}\right)=\phi\left(a_{i}\right)$ otherwise.

Suppose $a_{j}=2$ (then $j=1$ and we are in the setting of (7-6) and (7-7)). Denote Jord $d^{\prime}=$ Jord $\backslash\{(\rho, 2)\}$. In this case take $\phi^{\prime}$ to be the restriction of $\phi$.

Now the representation

$$
\begin{aligned}
\sigma_{1}^{\prime}=\delta\left(\left[\nu^{\left(\phi\left(a_{j}\right)+1\right) / 2} \rho, \nu^{\left(a_{j}-1\right) / 2-1} \rho\right]\right) & \\
& \times\left(\prod_{i=j+1}^{k} \delta\left(\left[\nu^{\left(\phi\left(a_{i}\right)+1\right) / 2} \rho, \nu^{\left(a_{i}-1\right) / 2} \rho\right]\right)\right) \rtimes \pi_{c u s p}
\end{aligned}
$$

contains by Lemma 4.1 a unique irreducible subrepresentation $\pi^{\prime}=\pi_{\left(\text {Jord }^{\prime}, \pi_{\text {cusp }}, \phi^{\prime}\right)}$. By the inductive assumption, $\pi^{\prime}$ is strongly positive (square integrable representation).

Note that $\sigma_{1}=\nu^{\left(a_{j}-1\right) / 2} \rho \rtimes \sigma_{1}^{\prime}$, and that this representation by (i) of Lemma 7.2 contains a unique irreducible subrepresentation. Clearly $\nu^{\left(a_{j}-1\right) / 2} \rho \rtimes \pi^{\prime} \hookrightarrow$ $\nu^{\left(a_{j}-1\right) / 2} \rho \rtimes \sigma_{1}^{\prime}$. Since $\sigma_{0} \hookrightarrow \nu^{\left(a_{j}-1\right) / 2} \rho \rtimes \sigma_{1}^{\prime}$ and $\pi \hookrightarrow \sigma_{0}$, we get

$$
\pi \hookrightarrow \nu^{\left(a_{j}-1\right) / 2} \rho \rtimes \pi^{\prime} .
$$

This implies

$$
s_{G L}(\pi) \leq\left(\nu^{\left(a_{j}-1\right) / 2} \rho+\nu^{-\left(a_{j}-1\right) / 2} \rho\right) \times s_{G L}\left(\pi^{\prime}\right)
$$

Suppose that $\pi^{\prime}$ is cuspidal. Then

$$
\phi\left(a_{j}\right)=a_{j}-2 \text { and } j=k .
$$

Thus, $\pi \hookrightarrow \nu^{\left(a_{k}-1\right) / 2} \rho \rtimes \pi_{c u s p}$. Suppose that we are in the even case. If $\epsilon\left(a_{1}\right)=1$, then $k$ must be 1 , and then $a_{1}=2$. The reducibility is at $1 / 2$ by (BA). This implies that the irreducible subrepresentation of $\nu^{1 / 2} \rho \rtimes \pi_{\text {cusp }}$ is square integrable and strongly positive. Suppose $\epsilon\left(a_{1}\right)=-1$. Then $a_{k}=2 k+2$. Now we have reducibility at $(2 k+1) / 2=\left(a_{k}-1\right) / 2$. Therefore, we again get that $\pi$ is strongly positive. Suppose that we are in the odd case. Now (7-16) and the definition of $j$ imply that we have reducibility at $((2 k-1)+1) / 2=k=\left(a_{k}-1\right) / 2$. Thus, $\pi$ is again strongly positive.

It remains to consider the case of non-cuspidal $\pi^{\prime}$. There are two possibilities.

Assume $a_{j}>\phi(j)+2$. Thus $a_{j} \geq \phi(j)+4$ (note that $a_{j-1}=\phi\left(a_{j-1}\right)$ if $j>1)$. If $a_{j}>4$, define $\operatorname{Jord}^{\prime \prime}=\left(\right.$ Jord $\left.\backslash\left\{\left(\rho, a_{j}\right)\right\}\right) \cup\left\{\left(\rho, a_{j}-4\right)\right\}$, and define $\phi^{\prime \prime}$ by $\phi^{\prime \prime}\left(a_{j}-4\right)=\phi\left(a_{j}\right)$ and $\phi^{\prime \prime}\left(a_{i}\right)=\phi\left(a_{i}\right)$ otherwise. If $a_{j}=\phi(j)+4$, define Jord $^{\prime \prime}=$ Jord $\backslash\left\{\left(\rho, a_{j}\right)\right\}$ and take $\phi^{\prime \prime}$ to be the restriction of $\phi$. 
Now the representation

$$
\begin{aligned}
\sigma_{2}^{\prime}=\delta\left(\left[\nu^{\left(\phi\left(a_{j}\right)+1\right) / 2} \rho, \nu^{\left(a_{j}-1\right) / 2-2} \rho\right]\right) & \\
& \times\left(\prod_{i=j+1}^{k} \delta\left(\left[\nu^{\left(\phi\left(a_{i}\right)+1\right) / 2} \rho, \nu^{\left(a_{i}-1\right) / 2} \rho\right]\right)\right) \rtimes \pi_{c u s p}
\end{aligned}
$$

contains a unique irreducible subrepresentation $\pi^{\prime \prime}=\pi_{\left(J o r d^{\prime \prime}, \pi_{\text {cusp }}, \phi^{\prime \prime}\right)}$. By the inductive assumption, $\pi^{\prime \prime}$ is strongly positive. Since $\sigma_{0} \hookrightarrow \delta\left(\left[\nu^{\left(a_{j}-1\right) / 2-1} \rho, \nu^{\left(a_{j}-1\right) / 2} \rho\right]\right)$ $\rtimes \sigma_{2}^{\prime}, \delta\left(\left[\nu^{\left(a_{j}-1\right) / 2-1} \rho, \nu^{\left(a_{j}-1\right) / 2} \rho\right]\right) \rtimes \pi^{\prime \prime} \hookrightarrow \delta\left(\left[\nu^{\left(a_{j}-1\right) / 2-1} \rho, \nu^{\left(a_{j}-1\right) / 2} \rho\right]\right) \rtimes \sigma_{2}^{\prime}, \pi \hookrightarrow \sigma_{0}$ and $\sigma_{2}=\delta\left(\left[\nu^{\left(a_{j}-1\right) / 2-1} \rho, \nu^{\left(a_{j}-1\right) / 2} \rho\right]\right) \rtimes \sigma_{2}^{\prime}$ contains a unique irreducible subrepresentation, we get

$$
\pi \hookrightarrow \delta\left(\left[\nu^{\left(a_{j}-1\right) / 2-1} \rho, \nu^{\left(a_{j}-1\right) / 2} \rho\right]\right) \rtimes \pi^{\prime \prime} .
$$

This implies

$$
\begin{aligned}
s_{G L}(\pi) \leq\left(\delta \left(\left[\nu^{\left(a_{j}-1\right) / 2-1} \rho,\right.\right.\right. & \left.\left.\nu^{\left(a_{j}-1\right) / 2} \rho\right]\right)+\nu^{-\left(a_{j}-1\right) / 2-1} \rho \times \nu^{\left(a_{j}-1\right) / 2} \rho \\
& \left.+\delta\left(\left[\nu^{-\left(a_{j}-1\right) / 2} \rho, \nu^{-\left(a_{j}-1\right) / 2+1} \rho\right]\right)\right) \times s_{G L}\left(\pi^{\prime \prime}\right) .
\end{aligned}
$$

Since $\pi^{\prime}$ and $\pi^{\prime \prime}$ are strongly positive, (7-19) applied to (7-16) gives

$$
s_{G L}(\pi) \leq \nu^{\left(a_{j}-1\right) / 2} \rho \times s_{G L}\left(\pi^{\prime}\right) .
$$

Since $\pi^{\prime}$ is strongly positive, (7-20) implies that $\pi$ is strongly positive.

It remains to consider the case $a_{j}=\phi\left(a_{j}\right)+2$. Since $\pi^{\prime}$ is not cuspidal, we have $j<k$.

Suppose $a_{j+1}=\phi\left(a_{j+1}\right)+2$. Note that $a_{j+1}=\phi\left(a_{j+1}\right)+2=\phi\left(a_{j}\right)+4=$ $a_{j}+2$. Then in the same way as we defined the strongly positive representation $\pi^{\prime}$ from $\pi$, we can repeat that construction and get that there exists an irreducible subrepresentation $\pi^{\prime \prime \prime}$ of

$$
\sigma_{3}^{\prime}=\left(\prod_{i=j+2}^{k} \delta\left(\left[\nu^{\left(\phi\left(a_{i}\right)+1\right) / 2} \rho, \nu^{\left(a_{i}-1\right) / 2} \rho\right]\right)\right) \rtimes \pi_{c u s p},
$$

which is strongly positive by the inductive assumption, and that

$$
\pi^{\prime} \hookrightarrow \nu^{\left(a_{j}+1\right) / 2} \rho \rtimes \pi^{\prime \prime \prime},
$$

which implies

$$
\begin{aligned}
\pi \hookrightarrow \nu^{\left(a_{j}-1\right) / 2} \rho \rtimes \pi^{\prime} \hookrightarrow \nu^{\left(a_{j}-1\right) / 2} \rho \times \nu^{\left(a_{j}+1\right) / 2} \rho \rtimes \pi^{\prime \prime \prime} & \\
& \hookrightarrow \nu^{\left(a_{j}-1\right) / 2} \rho \times \nu^{\left(a_{j}+1\right) / 2} \rho \rtimes \sigma_{3}^{\prime} .
\end{aligned}
$$

Since

$$
\mathfrak{s}\left(\left[\nu^{\left(a_{j}-1\right) / 2} \rho, \nu^{\left(a_{j}-1\right) / 2+1} \rho\right]\right) \rtimes \pi^{\prime \prime \prime} \hookrightarrow \nu^{\left(a_{j}-1\right) / 2} \rho \times \nu^{\left(a_{j}-1\right) / 2+1} \rho \rtimes \sigma_{3}^{\prime}
$$

( $\mathfrak{s}$ is defined in section 1 and is the Speh module) and $\sigma_{3}=\nu^{\left(a_{j}-1\right) / 2} \rho \times \nu^{\left(a_{j}+1\right) / 2} \rho \rtimes$ $\sigma_{3}^{\prime}$ has a unique irreducible subrepresentation by (iii) of Lemma 7.2 , we get

$$
\pi \hookrightarrow \mathfrak{s}\left(\left[\nu^{\left(a_{j}-1\right) / 2} \rho, \nu^{\left(a_{j}-1\right) / 2+1} \rho\right]\right) \rtimes \pi^{\prime \prime \prime} .
$$


This implies

$$
\begin{aligned}
s_{G L}(\pi) \leq\left(\mathfrak{s}\left(\left[\nu^{\left(a_{j}-1\right) / 2} \rho, \nu^{\left(a_{j}-1\right) / 2+1} \rho\right]\right)+\nu^{-\left(a_{j}-1\right) / 2-1} \rho \times \nu^{\left(a_{j}-1\right) / 2} \rho\right. \\
\left.+\mathfrak{s}\left(\left[\nu^{-\left(a_{j}-1\right) / 2-1} \rho, \nu^{-\left(a_{j}-1\right) / 2} \rho\right]\right)\right) \rtimes s_{G L}\left(\pi^{\prime \prime \prime}\right) .
\end{aligned}
$$

From this and (7-16) we again get (7-20) (using that $\pi^{\prime \prime \prime}$ is strongly positive). Thus, $\pi$ is strongly positive.

It remains to consider the case of $a_{j+1}>\phi\left(a_{j+1}\right)+2=\phi\left(a_{j}\right)+4=a_{j}+2$. Note that $\pi$ embeds into $\sigma_{3}$, which is isomorphic to

$$
\begin{aligned}
\nu^{\left(a_{j+1}-1\right) / 2} \rho \times \nu^{\left(a_{j}-1\right) / 2} \rho & \times \delta\left(\left[\nu^{\left(\phi\left(a_{j+1}\right)+1\right) / 2} \rho, \nu^{\left(a_{j+1}-1\right) / 2-1} \rho\right]\right) \\
& \times\left(\prod_{i=j+2}^{k} \delta\left(\left[\nu^{\left(\phi\left(a_{i}\right)+1\right) / 2} \rho, \nu^{\left(a_{i}-1\right) / 2} \rho\right]\right)\right) \rtimes \pi_{c u s p}
\end{aligned}
$$

(since $a_{j+1}>a_{j}+2$ ). Recall that $\sigma_{3}$ (and also the above representation) has a unique irreducible subrepresentation by (iii) of Lemma 7.2. Consider $J$ ord $^{\prime \prime \prime \prime}=$ $\left(\right.$ Jord $\left.\backslash\left\{\left(\rho, a_{j+1}\right)\right\}\right) \cup\left\{\left(\rho, a_{j+1}-2\right)\right\}$ and $\phi^{\prime \prime \prime \prime \prime}$ defined by $\phi^{\prime \prime \prime \prime}\left(a_{j+1}-2\right)=\phi\left(a_{j+1}\right)$ and $\phi^{\prime \prime \prime \prime}\left(a_{i}\right)=\phi\left(a_{i}\right)$ otherwise. By Lemma 4.1 we know that the representation

$$
\begin{aligned}
& \sigma_{3}^{\prime \prime}=\nu^{\left(a_{j}-1\right) / 2} \rho \times \delta\left(\left[\nu^{\left(\phi\left(a_{j+1}\right)+1\right) / 2} \rho, \nu^{\left(a_{j+1}-1\right) / 2-1} \rho\right]\right) \\
& \times\left(\prod_{i=j+2}^{k} \delta\left(\left[\nu^{\left(\phi\left(a_{i}\right)+1\right) / 2} \rho, \nu^{\left(a_{i}-1\right) / 2} \rho\right]\right)\right) \rtimes \pi_{c u s p}
\end{aligned}
$$

has a unique irreducible subrepresentation, which we denote by $\pi^{\prime \prime \prime \prime}$. Further, the inductive assumption implies that $\pi^{\prime \prime \prime \prime}$ is strongly positive. Since $\nu^{\left(a_{j}-1\right) / 2} \rho \rtimes$ $\pi^{\prime \prime \prime \prime} \hookrightarrow \nu^{\left(a_{j}-1\right) / 2} \rho \rtimes \sigma_{3}^{\prime \prime} \cong \sigma_{3}, \pi \hookrightarrow \sigma_{0} \hookrightarrow \sigma_{3}$ and $\sigma_{3}$ has a unique irreducible subrepresentation, we get

$$
\pi \hookrightarrow \nu^{\left(a_{j+1}-1\right) / 2} \rho \rtimes \pi^{\prime \prime \prime \prime} .
$$

This implies

$$
s_{G L}(\pi) \leq\left(\nu^{\left(a_{j+1}-1\right) / 2} \rho+\nu^{-\left(a_{j+1}-1\right) / 2} \rho\right) \times s_{G L}\left(\pi^{\prime \prime \prime \prime}\right) .
$$

Since $\left(a_{j+1}-1\right) / 2 \neq\left(a_{j}-1\right) / 2$, the above inequality and (7-16) imply (7-20) (using that $\pi^{\prime \prime \prime \prime}$ is strongly positive). Thus, $\pi$ is strongly positive. Now the proof of the square integrability claimed in the proposition is complete.

We no longer assume that condition (L) from the fifth section holds for our admissible triple. Denote

$$
\sigma=\left(\prod_{\rho}\left(\prod_{a \in J_{o r d}} \delta\left(\left[\nu^{\left(\phi_{\rho}(a)+1\right) / 2} \rho, \nu^{(a-1) / 2} \rho\right]\right)\right)\right) \rtimes \pi_{c u s p},
$$

where the first product runs over all $\rho$ for which $(\rho, a) \in$ Jord for some $a \in \mathbb{N}$, and the second product is taken in an order which follows the ordering of $\operatorname{Jord}_{\rho}$. Then in a similar way as in Lemma 4.1, it follows that $\sigma$ has a unique irreducible subrepresentation. Denote it by $\pi=\pi_{\left(J o r d, \pi_{c u s p}, \epsilon\right)}$. Now Proposition 7.1 implies that $\pi$ is square integrable. There are several arguments for that. Maybe the most elementary is the simple elementary Lemma 4.7 of [T4]. 
7.3. Lemma. Let Jord, $\pi_{\text {cusp }}, \epsilon$ be an admissible triple of alternated type. We have $\left(\pi_{\left(J o r d, \pi_{c u s p}, \epsilon\right)}\right)_{c u s p}=\pi_{\text {cusp }}$. Denote $\pi=\pi_{\left(J o r d, \pi_{c u s p}, \epsilon\right)}$. Then

$$
\left(\operatorname{Jord}(\pi), \pi_{c u s p}, \epsilon_{\pi}\right)=\left(\operatorname{Jord}, \pi_{c u s p}, \epsilon\right) .
$$

Proof. It is easy to see from the proof that the partial cuspidal support of $\pi$ is $\pi_{\text {cusp }}$. The fact that $\operatorname{Jord}(\pi)$ coincides with $J$ ord is proved using 2.1 (i). Now the representation $\pi$ coincides with the strongly positive representation in 4.1 of [M2]. The strongly positive property is equivalent (see 5.3 of [M2]) to the fact that $\epsilon_{\pi}$ is alternated. One easily sees that the alternated partially defined function $\epsilon$ on Jord, such that $\left(J o r d, \pi_{c u s p}, \epsilon\right)$ is admissible if it exists, is unique. This implies $\epsilon_{\pi}=\epsilon$.

This ends the proof that each admissible triple of alternated type comes from a (strongly positive) square integrable representation. Therefore, for a proof of Theorem 6.1 we need to settle the case of admissible triples of mixed type. Before we proceed to the proof in this case, we shall prove a useful (essentially combinatorial) result in the following section.

The simplest examples of the strongly positive irreducible square integrable representations are Steinberg representations. Further simple examples of such representations can be found in [T1]. Let $\pi$ be a strongly positive irreducible square integrable representation. Then it is easy to show that $s_{G L}(\pi)$ is irreducible (one can also write directly the Langlands parameters of these Jacquet modules). Other Jacquet modules do not need to always be irreducible (for the difference of the case of Steinberg representations). A very interesting representation from the point of view of the unitary duals of general linear groups can be tensor factors in $s_{G L}(\pi)$.

\section{TEMPERED AND SQUARE INTEGRABLE REPRESENTATIONS WITH THE SAME INFINITESIMAL CHARACTER}

Let Jord, $\pi_{c u s p}, \epsilon$ be an admissible triple of alternated type and let $\pi$ be the strongly positive representations with these invariants. Suppose

$$
\pi \leq \tau_{1} \times \cdots \times \tau_{n} \rtimes \pi_{c u s p},
$$

where $\tau_{i}$ are irreducible cuspidal representations. Then by induction with respect to the number of elements in $\operatorname{Jord} \backslash \operatorname{Jord}\left(\pi_{\text {cusp }}\right)$ we get

$$
\operatorname{Supp}(J \text { ord })=\operatorname{Supp}(\operatorname{Jord}(\pi))=\operatorname{Supp}\left(\operatorname{Jord}\left(\pi_{\text {cusp }}\right)\right)+\sum_{i=1}^{n}\left\{\tau_{i}, \check{\tau}_{i}\right\}
$$

Suppose now that the triple Jord, $\pi_{c u s p}, \epsilon$ is of mixed type. Then there exists a sequence of triples $\operatorname{Jord}_{i}, \pi_{\text {cusp }}, \epsilon_{i}, 1 \leq i \leq k$, such that: $\left(\operatorname{Jord}_{1}, \pi_{\text {cusp }}, \epsilon\right)=$ $\left(\operatorname{Jord}_{1}, \pi_{\text {cusp }}, \epsilon_{1}\right), \operatorname{Jord}_{i+1}, \pi_{\text {cusp }}, \epsilon_{i+1}$ is subordinated to $\operatorname{Jord}_{i}, \pi_{\text {cusp }}, \epsilon_{i}$ for $1 \leq i \leq$ $k-1$, and $J_{o r d}, \pi_{c u s p}, \epsilon_{k}$ is admissible of alternated type. Therefore, there exists $a(i), a(i)_{-} \in\left(\operatorname{Jord}_{i}\right)_{\rho_{i}}, i=1, \ldots, k-1$, such that

$$
\operatorname{Jord}_{i+1}=\operatorname{Jord}_{i} \backslash\left\{\left(\rho_{i}, a(i)\right),\left(\rho_{i}, a(i)_{-}\right)\right\}, \quad i=1, \ldots, k-1 .
$$


Denote by $\pi_{+}$the strongly positive representation determined by $\operatorname{Jord}_{k}, \pi_{c u s p}, \epsilon_{k}$. Now

$$
\begin{aligned}
& \operatorname{Supp}(\text { Jord }) \\
& =\operatorname{Supp}\left(\pi_{+}\right)+\sum_{i=1}^{k-1}\left(\delta\left(\left[\nu^{-(a(i)-1) / 2} \rho, \nu^{(a(i)-1) / 2} \rho\right]\right)\right. \\
& \left.+\delta\left(\left[\nu^{-\left(a(i)_{-}-1\right) / 2} \rho, \nu^{(a(i)--1) / 2} \rho\right]\right)\right) \\
& =\operatorname{Supp}\left(\pi_{+}\right)+\sum_{i=1}^{k-1}\left(\delta\left(\left[\nu^{-\left(a(i)_{-}-1\right) / 2} \rho, \nu^{(a(i)-1) / 2} \rho\right]\right)\right. \\
& \left.+\delta\left(\left[\nu^{-(a(i)-1) / 2} \rho, \nu^{(a(i)--1) / 2} \rho\right]\right)\right) .
\end{aligned}
$$

Suppose that $\pi_{+} \leq \tau_{1} \times \cdots \times \tau_{n} \rtimes \pi_{c u s p}$, where $\tau_{i}$ are irreducible cuspidal representations. Consider the following element of the Grothendieck group:

$$
\Pi=\left(\prod_{i=1}^{k-1}\left(\prod_{\left.j_{i}=-(a(i))_{-}-1\right) / 2}^{(a(i)-1) / 2} \nu^{j_{i}} \rho_{i}\right)\right) \times \tau_{1} \times \cdots \times \tau_{n} \rtimes \pi_{\text {cusp }} .
$$

Write

$$
\Pi=\sigma_{1} \times \cdots \times \sigma_{m} \rtimes \pi_{c u s p},
$$

where $\sigma_{i}$ are irreducible cuspidal representations (this is just another way of writing $(8-5)$ ). Then (8-2) and (8-4) imply

$$
\operatorname{Supp}(\text { Jord })=\operatorname{Supp}\left(\operatorname{Jord}\left(\pi_{\text {cusp }}\right)\right)+\sum_{i=1}^{m}\left\{\sigma_{i}, \check{\sigma}_{i}\right\} .
$$

One reconstructs Jord from $\operatorname{Supp}(J$ ord $)$ in the following simple way. One can write $\operatorname{Supp}(J$ ord $)=\sum_{i=1}^{l} \Delta_{i}$ as a sum of segments in such a way that among segments $\Delta_{i}$ there is no linking $\left(\Delta_{i}\right.$ are sets, so one can consider them as multisets). First,

$$
\Delta_{i} \neq \Delta_{j} \text { for } i \neq j .
$$

Write $\Delta_{i}=\left[\nu^{-\left(b_{i}-1\right) / 2} \gamma_{i}, \nu^{\left(b_{i}-1\right) / 2} \gamma_{i}\right]$. Then

$$
\text { Jord }=\left\{\left(\gamma_{1}, b_{1}\right), \ldots,\left(\gamma_{l}, b_{l}\right)\right\} .
$$

The following essentially combinatorial lemma will be very useful to us.

8.1. Lemma. Let the notation be as above. Then if $\pi$ is an irreducible tempered representation and $\pi \leq \Pi$, then $\pi$ is square integrable.

Proof. Suppose that $\pi$ is not square integrable. Then there exist irreducible unitarizable square integrable representations $\delta\left(\Delta_{i}\right)$ of $G L\left(n_{i}, F^{\prime}\right)$ for $i=1, \ldots, s$, where $s \geq 1$ and all $n_{i} \geq 1$, and an irreducible unitarizable square integrable representation $\pi^{\prime}$ of $S_{q^{\prime}}$ such that

$$
\pi \leq \delta\left(D_{1}\right) \times \cdots \times \delta\left(D_{s}\right) \rtimes \pi^{\prime} .
$$


Since $\pi \leq \Pi$, we get that $\check{\Delta}_{i}=\Delta_{i}$ for $i=1, \ldots, s$. From formula (8-7) we get

$$
\operatorname{Supp}(J \text { ord })=\operatorname{Supp}\left(\operatorname{Jord}\left(\pi^{\prime}\right)\right)+\sum_{i=1}^{s}\left(\Delta_{i}+\check{\Delta}_{i}\right)=\operatorname{Supp}\left(\operatorname{Jord}\left(\pi^{\prime}\right)\right)+2 \sum_{i=1}^{s} \Delta_{i} .
$$

Since $\pi^{\prime}$ is square integrable, from [M2 it follows that $\operatorname{Jord}\left(\pi^{\prime}\right), \pi_{\text {cusp }}, \epsilon_{\pi^{\prime}}$ form an admissible triple. Thus for $\operatorname{Jord}\left(\pi^{\prime}\right)$, the properties which we described above for Jord hold. Now (8-10) implies that (8-8) does not hold. This contradiction completes the proof.

We shall not use the following direct consequence of the above lemma, but it is interesting to note.

8.2. Corollary. Let $\tau_{1}$ and $\tau_{2}$ be irreducible tempered representations of $S_{q}$ with the same infinitesimal character. Then $\tau_{1}$ is square integrable if and only if $\tau_{2}$ is square integrable.

\section{Square integrability I}

In this and the following two sections, $\pi$ will denote an irreducible square integrable representation of $S_{q}, \rho$ will be an irreducible cuspidal $F^{\prime} / F$-selfdual representation of $G L\left(p, F^{\prime}\right)$, and $a, a_{-} \in \mathbb{N}$ will be such that $a-a_{-} \in 2 \mathbb{N}$. We shall assume that $(\rho, a)$ satisfies $(\mathrm{J}-1)$ and that

$$
\left[a_{-}, a\right] \cap \operatorname{Jord}_{\rho}(\pi)=\emptyset .
$$

Then by Corollary 4.7, the representation

$$
\delta\left(\left[\nu^{-\left(a_{-}-1\right) / 2} \rho, \nu^{(a-1) / 2} \rho\right]\right) \rtimes \pi
$$

has exactly two irreducible subrepresentations. They are not equivalent. Write

$$
\delta\left(\left[\nu^{-\left(a_{-}-1\right) / 2} \rho, \nu^{\left(a_{-}-1\right) / 2} \rho\right]\right) \rtimes \pi=T_{1} \oplus T_{-1}
$$

as a sum of two irreducible (tempered) representations $\left(T_{1}\right.$ and $T_{-1}$ are not equivalent). Then

$$
\delta\left(\left[\nu^{\left(a_{-}-1\right) / 2+1} \rho, \nu^{(a-1) / 2} \rho\right]\right) \rtimes T_{\eta}
$$

contains a unique irreducible subrepresentation, which we denote by $\pi_{\eta}$. Further, $\pi_{1} \neq \pi_{-1}$, and these representations are the irreducible subrepresentations of (9-2). Note that

$$
\begin{aligned}
\pi_{\eta} \hookrightarrow \delta\left(\left[\nu^{\left(a_{-}-1\right) / 2+1} \rho, \nu^{(a-1) / 2} \rho\right]\right) \rtimes T_{\eta} \\
\qquad \nu^{(a-1) / 2} \rho \times \nu^{(a-1) / 2-1} \rho \times \cdots \times \nu^{\left(a_{-}-1\right) / 2+1} \rho \rtimes T_{\eta} .
\end{aligned}
$$

Denote

$$
\Pi_{\eta}=\nu^{(a-1) / 2} \rho \times \nu^{(a-1) / 2-1} \rho \times \cdots \times \nu^{\left(a_{-}-1\right) / 2+1} \rho \rtimes T_{\eta} .
$$

Then $\Pi_{\eta}$ also has a unique irreducible subrepresentation by Lemma 4.5 (use (9-1)).

The aim of this and the following two sections is to prove the following

9.1. Proposition. Representations $\pi_{\eta}$ are square integrable. 
The importance of the above proposition follows from the following

9.2. Lemma. (i) Suppose that the claim of the above proposition holds for all $\pi, \rho, a$ and $a_{-}$as above, which satisfy

$$
\operatorname{deg}(\operatorname{Jord}(\pi))+p\left(a+a_{-}\right) / 2 \leq n .
$$

Then for each admissible triple Jord, $\pi_{\text {cusp }}, \epsilon$ of degree $\leq n$, there exist a positive integer $\ell$ and an irreducible square integrable representation of $S_{\ell}$ with these invariants.

(ii) Assume that the equality in (A) holds (besides (BA)) and suppose that the claim of the above proposition holds for all $\pi, \rho, a$ and $a_{-}$as above, which satisfy

$$
q+p\left(a+a_{-}\right) / 2 \leq n .
$$

Then for each admissible triple Jord, $\pi_{\text {cusp }}, \epsilon$ of degree $\leq n$, there exist a positive integer $\ell \leq n$ and an irreducible square integrable representation of $S_{\ell}$ with these invariants.

It is very easy to see that the above lemma implies that Theorem 6.1 follows from Proposition 9.1.

Proof. We shall prove the lemma by induction with respect to $n$. The proof of (i) and (ii) goes similarly, except that for (ii) one additionally uses, besides the inductive assumption, (2-1) (and the equality in (A)). The basis of induction is provided by Proposition 7.1. Let Jord, $\pi_{c u s p}, \epsilon$ be an admissible triple of degree $\leq n$. If it is of alternated type, then Proposition 7.1 implies the existence of an irreducible square integrable representation with these invariants.

Suppose now that Jord, $\pi_{\text {cusp }}, \epsilon$ is of mixed type. Then we can choose $a, a_{-} \in$ $\operatorname{Jord}_{\rho}$ for some $\rho$, such that $\epsilon(\rho, a)=\epsilon\left(\rho, a_{-}\right)$. Denote $\operatorname{Jord}^{\prime}=J$ ord $\backslash\left\{(\rho, a),\left(\rho, a_{-}\right)\right\}$. Let $\epsilon^{\prime}$ be the restriction of $\epsilon$ to $\operatorname{Jord}^{\prime}$ ( $\epsilon^{\prime}$ is partially defined). Then the degree of $J o r d^{\prime}$ is strictly lower than the degree of Jord, which is $\leq n$. Since $J o r d^{\prime}, \pi_{\text {cusp }}, \epsilon^{\prime}$ is an admissible triple, the inductive assumption implies that there exists an irreducible square integrable representation $\pi^{\prime}$ with these invariants. Now, the assumption of the lemma implies that $\pi_{1}$ and $\pi_{-1}$ are square integrable. Clearly, $\left(\pi_{\eta}\right)_{\text {cusp }}=\pi_{\text {cusp }}$ for $\eta= \pm 1$. Further, Lemma 5.2 of [M2] implies $\operatorname{Jord}\left(\pi_{\eta}\right)=$ Jord for $i= \pm 1$. Therefore, the first two invariants of $\pi_{1}$ and $\pi_{-1}$ are the same. Since $\pi_{1} \neq \pi_{-1}$, we have $\epsilon_{\pi_{1}} \neq \epsilon_{\pi_{-1}}$ (this implies the main result of [M2], the injectivity of (2-2)). Further, by (9-1) we can apply Lemma 5.4, Lemma 5.5 and (ii) of Proposition 6.1 (all) from [M2]. They give that the restriction of $\epsilon_{\pi_{\eta}}, \eta= \pm 1$, to $\operatorname{Jord}\left(\pi^{\prime}\right)=\operatorname{Jord}^{\prime}$ is $\epsilon_{\pi^{\prime}}=\epsilon^{\prime}$.

It is easy to see that $\epsilon^{\prime}$ can be extended to a partially defined function on Jord, to make an admissible triple with Jord and $\pi_{c u s p}$, exactly in two ways. Denote these extensions by $\epsilon_{1}$ and $\epsilon_{-1}$. The above discussion implies $\epsilon_{\pi_{\eta}}=\epsilon_{\eta}$ for $\eta= \pm 1$ or $\epsilon_{\pi_{\eta}}=\epsilon_{-\eta}$ for $\eta= \pm 1$. Since $\epsilon \in\left\{\epsilon_{ \pm 1}\right\}$, we get that there exists $\eta \in\{ \pm 1\}$ such that $\epsilon_{\pi_{\eta}}=\epsilon$. This completes the proof.

Now we shall start the proof of Proposition 9.1.

To prove the proposition, it is enough to prove it for $\pi$ which satisfy (L). There are several ways to see this. Technically, the simplest way seems to be to apply an elementary Lemma 4.7 of [T4].

Note that the proof of the proposition that we shall present actually does not require (L), but, assuming (L), the proof (which is relatively complicated) will become a little bit shorter and will use simpler notation. 
Therefore, we shall assume that $\pi$ satisfies (L) (this assumption is not important for this section, since it is automatically satisfied here).

First we shall prove the proposition for cuspidal $\pi$.

9.3. Lemma. If $\pi$ is cuspidal, then $\pi_{\eta}$ are square integrable.

Proof. We shall prove the lemma by induction with respect to $a-a_{-}$.

Suppose that $\sigma$ is an irreducible subquotient of (9-2). From (9-2) it follows that

$$
s_{G L}(\sigma) \leq\left(\sum_{i=-\left(a_{-}-1\right) / 2}^{(a-1) / 2+1} \delta\left(\left[\nu^{i} \rho, \nu^{(a-1) / 2} \rho\right]\right) \times \delta\left(\left[\nu^{-i+1} \rho, \nu^{\left(a_{-}-1\right) / 2} \rho\right]\right)\right) \otimes \pi_{c u s p} .
$$

This implies that the factors of $\sigma$ are contained in

$$
\left\{\nu^{-(a-1) / 2} \rho, \nu^{-(a-1) / 2+1} \rho, \ldots, \nu^{(a-1) / 2} \rho\right\} .
$$

Suppose that $\nu^{-(a-1) / 2} \rho$ is a factor of $\sigma$. Then we see from (9-6) that the only possibility of getting $\nu^{-(a-1) / 2} \rho$ as a factor is to take the index $i=(a-1) / 2$. This implies that $\delta\left(\left[\nu^{-(a-1) / 2} \rho, \nu^{\left(a_{-}-1\right) / 2} \rho\right]\right) \otimes \pi_{c u s p} \leq s_{G L}(\sigma)$. Now Lemma 4.4 implies that $\sigma$ is an irreducible (Langlands) quotient of (9-2). Since (9-2) reduces, we obtain that $\sigma \neq \pi_{\eta}$ for $\eta= \pm 1$.

Fix $\eta \in\{ \pm 1\}$. We have just shown that

the factors of $\pi_{\eta}$ are contained in $\left\{\nu^{-(a-1) / 2+1} \rho, \nu^{-(a-1) / 2+2} \rho, \ldots, \nu^{(a-1) / 2} \rho\right\}$.

Suppose $a=a_{-}+2$. Then $\pi_{\eta} \hookrightarrow \nu^{(a-1) / 2} \rho \rtimes T_{\eta}$. Now (1-2) implies

$$
s_{G L}\left(\pi_{\eta}\right) \leq s_{G L}\left(\nu^{(a-1) / 2} \rho \rtimes T_{\eta}\right)=\left(\nu^{(a-1) / 2} \rho+\nu^{-(a-1) / 2} \rho\right) \times s_{G L}\left(T_{\eta}\right) .
$$

Further, the above inequality and (9-7) imply

$$
s_{G L}\left(\pi_{\eta}\right) \leq \nu^{(a-1) / 2} \rho \times s_{G L}\left(T_{\eta}\right) .
$$

From this and Lemma 3.5, we get that $\pi_{\eta}$ is tempered (recall that $T_{\eta}$ is tempered). Lemma 8.1 implies now that $\pi_{\eta}$ is square integrable.

It remains to consider the case $a>a_{-}+2$. Consider the unique irreducible subrepresentation of $\delta\left(\left[\nu^{\left(a_{-}-1\right) / 2+1} \rho, \nu^{(a-1) / 2-1} \rho\right]\right) \rtimes T_{\eta}$, which we denote by $\pi_{\eta}^{\prime}$. By the inductive assumption, $\pi_{\eta}^{\prime}$ is square integrable. Since

$$
\nu^{(a-1) / 2} \rho \rtimes \pi_{\eta}^{\prime} \hookrightarrow \nu^{(a-1) / 2} \rho \rtimes \delta\left(\left[\nu^{\left(a_{-}-1\right) / 2+1} \rho, \nu^{(a-1) / 2-1} \rho\right]\right) \rtimes T_{\eta} \hookrightarrow \Pi_{\eta}
$$

and $\Pi_{\eta}$ has a unique irreducible subrepresentation, which is $\pi_{\eta}$, we get

$$
\pi_{\eta} \hookrightarrow \nu^{(a-1) / 2} \rho \rtimes \pi_{\eta}^{\prime} .
$$

Now, in the same way as above, from (9-7) we get

$$
s_{G L}\left(\pi_{\eta}\right) \leq \nu^{(a-1) / 2} \rho \times s_{G L}\left(\pi_{\eta}^{\prime}\right) .
$$

Lemma 3.5 implies the square integrability.

Additional information about representations $\pi_{\eta}$ and their Jacquet modules in the case of cuspidal $\pi$ can be found in [T3]. 


\section{Square Integrability II}

In this section, we shall continue to use the notation introduced in the last section.

For the proof of Proposition 9.1, it remains to show the square integrability of $\pi_{\eta}$ in the case of non-cuspidal $\pi$. We shall prove the proposition by induction. The basis of the induction is provided by Proposition 7.1. The inductive assumption is: the claim of Proposition 9.1 holds for all $\pi, \rho, a$ and $a_{-}$satisfying

$$
\left(a+a_{-}\right) / 2+\operatorname{deg} \operatorname{Jord}(\pi)<n .
$$

Then Lemma 9.2 implies that each admissible triple $J_{o r d,} \pi_{c u s p}, \epsilon$ of degree $<n$ corresponds to some square integrable representation.

In this section we shall suppose

$$
\left(a+a_{-}\right) / 2+\operatorname{deg} \operatorname{Jord}(\pi)=n .
$$

Denote

$$
\begin{aligned}
b=\max \left\{a^{\prime} \in \operatorname{Jord}_{\rho}(\pi) ; \quad a^{\prime}-2 \notin \operatorname{Jord}_{\rho}(\pi)\right. & \\
& \text { or } a^{\prime}-2 \in \operatorname{Jord}_{\rho}(\pi) \text { and } \epsilon_{\pi}\left(a^{\prime}\right)=\epsilon_{\pi}\left(a^{\prime}-2\right), \\
& \text { or } \left.a^{\prime}=2 \text { and } \epsilon_{\pi}\left(a^{\prime}\right)=1\right\} .
\end{aligned}
$$

Suppose that the set on the right-hand side of (10-1) is empty. This assumption first implies that $\pi$ is strongly positive. Further, this and (2-6) imply $\operatorname{Jord}_{\rho}(\pi)=$ $\operatorname{Jord}_{\rho}\left(\pi_{\text {cusp }}\right)$. Thus, $\phi\left(a_{i}\right)=a_{i}$ for all $i$. This implies that the representation (7-10) is cuspidal. Thus, $\pi$ must be cuspidal (see the seventh section). This contradicts our assumption. Therefore, since $\pi$ is not cuspidal, $b$ is well defined.

Now we shall consider all possible relations among $a, a_{-}$and $b$.

10.1. First we shall analyze the case

$$
b<a_{-} .
$$

The definition of $b$ implies

$$
\max \operatorname{Jord}_{\rho}(\pi) \leq a_{-}-2
$$

(if $m=\max \operatorname{Jord}_{\rho}(\pi) \geq a_{-}$, then (9-1) implies $m>a$, which implies that the set $\left\{a^{\prime} \in \operatorname{Jord}_{\rho}(\pi) ; a^{\prime}>a\right\}$ is non-empty; denote the minimum of it by $m^{\prime}$; then clearly $m^{\prime}-2 \notin \operatorname{Jord}_{\rho}(\pi)$, which implies $m^{\prime} \leq b$, and further $a_{-}<a<m^{\prime} \leq b$; this contradicts (10-2)).

From this it follows directly that:

factors of $\pi$ are contained in $\left\{\nu^{-\left(a_{-}-1\right) / 2+1} \rho, \nu^{-\left(a_{-}-1\right) / 2+2} \rho, \ldots, \nu^{\left(a_{-}-1\right) / 2-1} \rho\right\}$.

We can get this also from Lemma 5.2 (this lemma can give more precise information about factors).

First we shall prove the following

Lemma. Suppose $b<a_{-}$. Then $\pi_{\eta}$ has no factors in the set $X=\left\{\nu^{-(a-1) / 2-z} \rho\right.$, $\left.z \in \mathbb{Z}_{+}\right\}$. 
Proof. First note that (10-4) implies that $\pi$ has no factors in $X$. This, (1-2) and (1-4) imply that $\pi_{\eta}$ has no factors in the set $\left\{\nu^{-(a-1) / 2-z} \rho, z \in \mathbb{N}\right\}$. Therefore, it remains to show that $\nu^{-(a-1) / 2} \rho$ is not a factor of $\pi_{\eta}$. Suppose that it is a factor.

Since $\nu^{-(a-1) / 2-1} \rho$ is not a factor of $\pi_{\eta}$ by the above discussion, we can apply Lemma 3.4 to $\pi_{\eta}$ for $\tau=\nu^{-(a-1) / 2} \rho$. Then

$$
\pi_{\eta} \hookrightarrow \delta\left(\left[\nu^{-(a-1) / 2} \rho, \nu^{\left(a^{\prime}-1\right) / 2} \rho\right]\right) \rtimes \sigma
$$

for some irreducible representation $\sigma$ and for $a^{\prime} \in \mathbb{Z}$ such that $a+a^{\prime} \in 2 \mathbb{Z}$ and $\left(a^{\prime}-1\right) / 2-(-(a-1) / 2) \geq 0$ (i.e. $\left.a^{\prime} \geq-a+2\right)$. Now (10-5) implies that

$$
\nu^{\left(a^{\prime}-1\right) / 2} \rho \otimes \nu^{\left(a^{\prime}-1\right) / 2-1} \rho \otimes \cdots \otimes \nu^{-(a-1) / 2} \rho \otimes \rho_{1} \otimes \rho_{2} \otimes \cdots \otimes \rho_{l} \otimes \pi_{\text {cusp }}
$$

is an irreducible subquotient (actually a quotient) of a corresponding Jacquet module of $\pi_{\eta}$, for some irreducible cuspidal representations $\rho_{i}$.

Recall

$$
\pi_{\eta} \hookrightarrow \delta\left(\left[\nu^{-\left(a_{-}-1\right) / 2} \rho, \nu^{(a-1) / 2} \rho\right]\right) \rtimes \pi .
$$

We shall consider two possible relations of $a_{-}$and $a^{\prime}$. Suppose first

$$
a^{\prime} \leq a_{-} .
$$

The transitivity of Jacquet modules implies that (10-6) is a subquotient of a standard Jacquet module of $s_{\left(\left(a+a_{-}\right) / 2\right)}\left(\pi_{\eta}\right)$. Therefore, there exists an irreducible subquotient $\sigma \otimes \tau$ of $s_{\left(\left(a+a_{-}\right) / 2\right)}\left(\pi_{\eta}\right)$ such that (10-6) is a subquotient of a corresponding standard Jacquet module of $\sigma \otimes \tau$. Now $a^{\prime} \leq a_{-}$implies that $\nu^{-(a-1) / 2} \rho$ must be in the support of $\sigma$ (the support is defined in Proposition 1.10 of [Z], as we already noted).

Note that (10-7) implies

$$
\sigma \otimes \tau \leq s_{\left(\left(a+a_{-}\right) / 2\right)}\left(\pi_{\eta}\right) \leq s_{\left(\left(a+a_{-}\right) / 2\right)}\left(\delta\left(\left[\nu^{-\left(a_{-}-1\right) / 2} \rho, \nu^{(a-1) / 2} \rho\right]\right) \rtimes \pi\right) .
$$

Write

$$
\begin{aligned}
& \mu^{*}\left(\delta\left(\left[\nu^{-\left(a_{-}-1\right) / 2} \rho, \nu^{(a-1) / 2} \rho\right]\right) \rtimes \pi\right) \\
=\left(\sum_{i=-\left(a_{-}-1\right) / 2-1}^{(a-1) / 2} \sum_{j=i}^{(a-1) / 2} \delta\left(\left[\nu^{-i} \rho, \nu^{\left(a_{-}-1\right) / 2} \rho\right]\right)\right. & \times \delta\left(\left[\nu^{j+1} \rho, \nu^{(a-1) / 2} \rho\right]\right) \\
& \left.\otimes \delta\left(\left[\nu^{i+1} \rho, \nu^{j} \rho\right]\right)\right) \rtimes \mu^{*}(\pi) .
\end{aligned}
$$

Because of (10-4), the only terms in the above sum which can have an irreducible subquotient $\sigma^{\prime} \otimes \tau^{\prime}$ such that $\nu^{-(a-1) / 2} \rho$ is in the support of $\tau^{\prime}$ are

$$
\delta\left(\left[\nu^{-(a-1) / 2} \rho, \nu^{\left(a_{-}-1\right) / 2} \rho\right]\right) \times \sigma^{\prime \prime} \otimes \tau^{\prime \prime},
$$

where $\sigma^{\prime \prime} \otimes \tau^{\prime \prime} \leq \mu^{*}(\pi)$ (then we must have $i=j=(a-1) / 2$ in the above sum). Since $\sigma \otimes \tau$ is a subquotient of $s_{\left(\left(a+a_{-}\right) / 2\right)}\left(\pi_{\eta}\right)$, there is only one possibility for $\sigma^{\prime \prime} \otimes$ $\tau^{\prime \prime}$ : we must have $\sigma^{\prime \prime}=1$ and $\tau^{\prime \prime}=\pi$. Thus $\sigma \otimes \tau=\delta\left(\left[\nu^{-\left(a_{-}-1\right) / 2} \rho, \nu^{(a-1) / 2} \rho\right]\right) \otimes \pi$. Now $\pi_{\eta}$ is a Langlands quotient of $\delta\left(\left[\nu^{-(a-1) / 2} \rho, \nu^{\left(a_{-}-1\right) / 2} \rho\right]\right) \rtimes \pi$ by Lemma 4.4. This and (10-7) contradict the reducibility of $\delta\left(\left[\nu^{-(a-1) / 2} \rho, \nu^{\left(a_{-}-1\right) / 2} \rho\right]\right) \rtimes \pi$.

Therefore, we must have

$$
a_{-}<a^{\prime}
$$


Note that from the fact that (10-6) is a subquotient of a Jacquet module of $\pi_{\eta}$, Lemma 3.1 and Lemma 3.2 imply that

$$
\nu^{\left(a^{\prime}-1\right) / 2} \rho \otimes \gamma \leq s_{(p)}\left(\pi_{\eta}\right),
$$

for some irreducible $\gamma$, such that $\nu^{-(a-1) / 2} \rho$ is a factor of $\gamma$. Further, (10-7) implies

$$
\nu^{\left(a^{\prime}-1\right) / 2} \rho \otimes \gamma \leq s_{(p)}\left(\delta\left(\left[\nu^{-\left(a_{-}-1\right) / 2} \rho, \nu^{(a-1) / 2} \rho\right]\right) \rtimes \pi\right) .
$$

Write

$$
s_{(p)}(\pi)=\sum_{i} \mu_{i} \otimes \lambda_{i}
$$

as a sum of irreducible representations. Then (10-9) implies (recall that $\pi$ satisfies $(\mathrm{L}))$

$$
\begin{gathered}
s_{(p)}\left(\delta\left(\left[\nu^{-\left(a_{-}-1\right) / 2} \rho, \nu^{(a-1) / 2} \rho\right]\right) \rtimes \pi\right)=\sum_{i} \mu_{i} \otimes \delta\left(\left[\nu^{-\left(a_{-}-1\right) / 2} \rho, \nu^{(a-1) / 2} \rho\right]\right) \rtimes \lambda_{i} \\
(10-12) \quad+\nu^{(a-1) / 2} \rho \otimes \delta\left(\left[\nu^{-\left(a_{-}-1\right) / 2} \rho, \nu^{(a-1) / 2-1} \rho\right]\right) \rtimes \pi \\
(10-13) \quad+\nu^{\left(a_{-}-1\right) / 2} \rho \otimes \delta\left(\left[\nu^{-\left(a_{-}-1\right) / 2+1} \rho, \nu^{(a-1) / 2} \rho\right]\right) \rtimes \pi .
\end{gathered}
$$

Since $a_{-}<a^{\prime}$, it follows that $\nu^{\left(a^{\prime}-1\right) / 2} \rho \otimes \gamma$ cannot be a subquotient of (10-13). From (1-2), (1-4) and (10-4) we get that $\nu^{-(a-1) / 2} \rho$ is not a factor of $\delta\left(\left[\nu^{-\left(a_{-}-1\right) / 2} \rho, \nu^{(a-1) / 2-1} \rho\right]\right) \rtimes \pi$ (this term shows up in (10-12)). This implies that $\nu^{\left(a^{\prime}-1\right) / 2} \rho \otimes \gamma$ is not a subquotient of (10-12). Therefore, $\nu^{\left(a^{\prime}-1\right) / 2} \rho \otimes \gamma$ is a subquotient for some $i$ of $\mu_{i} \otimes \delta\left(\left[\nu^{-\left(a_{-}-1\right) / 2} \rho, \nu^{(a-1) / 2} \rho\right]\right) \rtimes \lambda_{i}$. This implies $\nu^{\left(a^{\prime}-1\right) / 2} \rho \cong \mu_{i}$. Now (10-11) and Lemma 3.6 imply $a^{\prime} \in \operatorname{Jord}_{\rho}(\pi)$. Since $a_{-}<a^{\prime}$, we get a contradiction with (10-3). This contradiction ends the proof.

We shall now prove square integrability of $\pi_{\eta}$ in the case $b<a_{-}$. Recall (9-4)

$$
\begin{aligned}
\pi_{\eta} \hookrightarrow \delta\left(\left[\nu^{\left(a_{-}-1\right) / 2+1} \rho, \nu^{(a-1) / 2} \rho\right]\right) \times T_{\eta} \\
\hookrightarrow \nu^{(a-1) / 2} \rho \times \delta\left(\left[\nu^{\left(a_{-}-1\right) / 2+1} \rho, \nu^{(a-1) / 2-1} \rho\right]\right) \times T_{\eta} .
\end{aligned}
$$

Suppose $a=a_{-}+2$. Then (10-14) implies

$$
s_{G L}\left(\pi_{\eta}\right) \leq\left(\nu^{(a-1) / 2} \rho+\nu^{-(a-1) / 2} \rho\right) \times s_{G L}\left(T_{\eta}\right) .
$$

Now the above lemma implies

$$
s_{G L}\left(\pi_{\eta}\right) \leq \nu^{(a-1) / 2} \rho \times s_{G L}\left(T_{\eta}\right) .
$$

Since $T_{\eta}$ is a tempered representation, Lemma 3.5 implies that $\pi_{\eta}$ is tempered. Now Lemma 8.1 implies that $\pi_{\eta}$ is square integrable.

Suppose now $a>a_{-}+2$. Then denote by $\pi_{\eta}^{\prime}$ the irreducible subrepresentation of

$$
\delta\left(\left[\nu^{\left(a_{-}-1\right) / 2+1} \rho, \nu^{(a-1) / 2-1} \rho\right]\right) \rtimes T_{\eta}
$$

(which is unique; see section 9). Then the inductive assumption implies that $\pi_{\eta}^{\prime}$ is square integrable. Since the representation $\Pi_{\eta}$ from (9-5) has a unique irreducible subrepresentation, and both $\pi_{\eta}$ and $\nu^{(a-1) / 2-1} \rho \rtimes \pi_{\eta}^{\prime}$ embed into it, we conclude that

$$
\pi_{\eta} \hookrightarrow \nu^{(a-1) / 2} \rho \rtimes \pi_{\eta}^{\prime} .
$$


Applying $s_{G L}$ to both sides and using (1-2), we get from the above lemma

$$
s_{G L}\left(\pi_{\eta}\right) \leq \nu^{(a-1) / 2} \rho \times s_{G L}\left(\pi_{\eta}^{\prime}\right) .
$$

Now Lemma 3.5 implies the square integrability of $\pi_{\eta}$. This ends the proof of the square integrability of $\pi_{\eta}$ in the case $b<a$.

It remains to consider the case $a_{-}<b$. The condition (9-1) implies $a<b$.

10.2. We shall now assume

$$
a<b .
$$

Then

$$
b>4 .
$$

10.2.1. First we shall consider the case

$$
b-2 \in \operatorname{Jord}_{\rho}(\pi) .
$$

Then clearly $b_{-}=b-2$. The definition (10-1) of $b$ implies

$$
\epsilon_{\pi}(b)=\epsilon_{\pi}\left(b_{-}\right) .
$$

Note that (10-19) and (9-1) imply

$$
a<b_{-} .
$$

Therefore,

$$
a+2<b .
$$

Note that (10-19) and the definition (10-1) of $b$ imply that $b$ is the maximum of all $a^{\prime} \in \operatorname{Jord}_{\rho}(\pi)$ such that $a_{-}^{\prime} \in \operatorname{Jord}_{\rho}(\pi)$ is defined and $e_{\pi}\left(a^{\prime}\right)=e_{\pi}\left(a_{-}^{\prime}\right)$. Therefore, Lemma 5.2 implies that

$$
\pi \text { has no factors in }\left\{\nu^{-(b-1) / 2-z} \rho ; z \in \mathbb{Z}_{+}\right\} .
$$

By Lemma 5.1, there exists an irreducible square integrable representation $\pi^{\prime}$ such that

$$
\pi \hookrightarrow \delta\left(\left[\nu^{-\left(b_{-}-1\right) / 2} \rho, \nu^{(b-1) / 2} \rho\right]\right) \rtimes \pi^{\prime} .
$$

Proposition 2.1 implies

$$
\operatorname{Jord}\left(\pi^{\prime}\right)=\operatorname{Jord}(\pi) \backslash\left\{(\rho, b),\left(\rho, b_{-}\right)\right\} .
$$

Further, (10-7), (10-22), and the facts $(a-1) / 2+1<(b-1) / 2, b=b_{-}+2$ and $(a-1) / 2 \leq\left(b_{-}-1\right) / 2\left(\right.$ which implies $\left.-\left(b_{-}-1\right) / 2 \leq-\left(a_{-}-1\right) / 2\right)$ imply

$$
\delta\left(\left[\nu^{-\left(a_{-}-1\right) / 2} \rho, \nu^{(a-1) / 2} \rho\right]\right) \times \nu^{(b-1) / 2} \rho \times \delta\left(\left[\nu^{-\left(b_{-}-1\right) / 2} \rho, \nu^{\left(b_{-}-1\right) / 2} \rho\right]\right) \rtimes \pi^{\prime}
$$

$$
\cong \nu^{(b-1) / 2} \rho \times \delta\left(\left[\nu^{-\left(b_{-}-1\right) / 2} \rho, \nu^{\left(b_{-}-1\right) / 2} \rho\right]\right) \times \delta\left(\left[\nu^{-\left(a_{-}-1\right) / 2} \rho, \nu^{(a-1) / 2} \rho\right]\right) \rtimes \pi^{\prime} .
$$

Now we know that $\delta\left(\left[\nu^{-\left(a_{-}-1\right) / 2} \rho, \nu^{(a-1) / 2} \rho\right]\right) \rtimes \pi^{\prime}$ has exactly two irreducible subrepresentations. Denote them by $\pi_{\eta^{\prime}}^{\prime}, \eta^{\prime} \in\{ \pm 1\}$. Thus

$$
\bigoplus_{\eta^{\prime} \in\{ \pm 1\}} \pi_{\eta^{\prime}}^{\prime} \hookrightarrow \delta\left(\left[\nu^{-\left(a_{-}-1\right) / 2} \rho, \nu^{(a-1) / 2} \rho\right]\right) \rtimes \pi^{\prime} .
$$


Applying the inductive assumption to $\pi_{\eta^{\prime}}^{\prime}$ (these representations satisfy the conditions of section 9), we get that they are square integrable. From Proposition 2.1, we get

$$
\begin{aligned}
\operatorname{Jord}\left(\pi_{\eta^{\prime}}^{\prime}\right)=\operatorname{Jord}\left(\pi^{\prime}\right) \cup & \left\{(\rho, a),\left(\rho, a_{-}\right)\right\} \\
& =\left(\operatorname{Jord}(\pi) \backslash\left\{(\rho, b),\left(\rho, b_{-}\right)\right\}\right) \cup\left\{(\rho, a),\left(\rho, a_{-}\right)\right\} .
\end{aligned}
$$

Since $b_{-} \notin \operatorname{Jord} d_{\rho}\left(\pi_{\eta^{\prime}}^{\prime}\right)$, the representation

$$
\delta\left(\left[\nu^{-\left(b_{-}-1\right) / 2} \rho, \nu^{\left(b_{-}-1\right) / 2} \rho\right]\right) \times \pi_{\eta^{\prime}}^{\prime}
$$

reduces into a sum of two irreducible tempered representations (which are not equivalent). Further, (10-27) implies that

$$
\bigoplus_{\eta^{\prime} \in\{ \pm 1\}} \nu^{(b-1) / 2} \times \delta\left(\left[\nu^{-\left(b_{-}-1\right) / 2} \rho, \nu^{\left(b_{-}-1\right) / 2} \rho\right]\right) \times \pi_{\eta^{\prime}}^{\prime}
$$

embeds into (10-26). The above discussion implies that (10-30) has at least four irreducible subrepresentations (since (10-29) reduces into a sum of two irreducible subrepresentation for each $\left.\eta^{\prime} \in\{ \pm 1\}\right)$.

Now we shall show that (10-26) has at most four irreducible subrepresentations (the above discussion will imply that it has exactly four irreducible subrepresentations). First recall that (10-26) is isomorphic to (10-25). Therefore, it is enough to see that (10-25) has at most four irreducible subrepresentations. Note that (10-25) embeds into

$$
\begin{aligned}
\nu^{(b-1) / 2} & \times \delta\left(\left[\nu^{\left(a_{-}-1\right) / 2+1} \rho, \nu^{(a-1) / 2} \rho\right]\right) \\
& \times \delta\left(\left[\nu^{-\left(b_{-}-1\right) / 2} \rho, \nu^{\left(b_{-}-1\right) / 2} \rho\right]\right) \times \delta\left(\left[\nu^{-\left(a_{-}-1\right) / 2} \rho, \nu^{\left(a_{-}-1\right) / 2} \rho\right]\right) \rtimes \pi^{\prime} \\
\hookrightarrow & \nu^{(b-1) / 2} \times \nu^{(a-1) / 2} \rho \times \nu^{(a-1) / 2-1} \rho \times \cdots \times \nu^{\left(a_{-}-1\right) / 2+1} \rho \\
& \times \delta\left(\left[\nu^{-\left(b_{-}-1\right) / 2} \rho, \nu^{\left(b_{-}-1\right) / 2} \rho\right]\right) \times \delta\left(\left[\nu^{-\left(a_{-}-1\right) / 2} \rho, \nu^{\left(a_{-}-1\right) / 2} \rho\right]\right) \rtimes \pi^{\prime} .
\end{aligned}
$$

It is enough to prove that (10-31) has at most four irreducible subrepresentations. Since each irreducible subrepresentation of (10-31) has

$$
\begin{aligned}
t= & \nu^{(b-1) / 2} \otimes \nu^{(a-1) / 2} \rho \otimes \nu^{(a-1) / 2-1} \rho \otimes \cdots \otimes \nu^{\left(a_{-}-1\right) / 2+1} \rho \\
& \otimes \delta\left(\left[\nu^{-\left(b_{-}-1\right) / 2} \rho, \nu^{\left(b_{-}-1\right) / 2} \rho\right]\right) \otimes \delta\left(\left[\nu^{-\left(a_{-}-1\right) / 2} \rho, \nu^{\left(a_{-}-1\right) / 2} \rho\right]\right) \otimes \pi^{\prime}
\end{aligned}
$$

as a quotient of a corresponding Jacquet module, the fact that (10-31) has at most four irreducible subrepresentations will follow from

Lemma. The multiplicity of $t$ (defined in (10-32)), in a corresponding standard Jacquet module of (10-31) is four. 
Proof. Denote the representation (10-31) by $\Psi$. Then

$$
\begin{gathered}
\mu^{*}(\Psi)=\left(1 \otimes \nu^{(b-1) / 2} \rho+\nu^{(b-1) / 2} \rho \otimes 1+\nu^{-(b-1) / 2} \rho \otimes 1\right) \\
\times\left(\prod_{i=\left(a_{-}-1\right) / 2+1}^{(a-1) / 2}\left(1 \otimes \nu^{i} \rho+\nu^{i} \rho \otimes 1+\nu^{-i} \rho \otimes 1\right)\right) \\
\times\left(\sum_{i^{\prime}=-\left(a_{-}-1\right) / 2-1}^{\left(a_{-}-1\right) / 2} \sum_{j^{\prime}=i^{\prime}}^{\left(a_{-}-1\right) / 2} \delta\left(\left[\nu^{-i^{\prime}} \rho, \nu^{\left(a_{-}-1\right) / 2} \rho\right]\right) \times \delta\left(\left[\nu^{j^{\prime}+1} \rho, \nu^{\left(a_{-}-1\right) / 2} \rho\right]\right)\right. \\
\times\left(\sum_{i^{\prime \prime}=-\left(b_{-}-1\right) / 2-1}^{\left(b_{-}-1\right) / 2} \sum_{j^{\prime \prime}=i^{\prime \prime}}^{\left(b_{-}-1\right) / 2} \delta\left(\left[\nu^{-i^{\prime \prime}} \rho, \nu^{\left(b_{-}-1\right) / 2} \rho\right]\right) \times \delta\left(\left[\nu^{j^{\prime \prime}+1} \rho, \nu^{\left(b_{-}-1\right) / 2} \rho\right]\right)\right. \\
\left.\otimes \delta\left(\left[\nu^{i^{\prime \prime}+1} \rho, \nu^{j^{\prime \prime}} \rho\right]\right)\right) \rtimes \mu^{*}\left(\pi^{\prime}\right) .
\end{gathered}
$$

If $t$ is a subquotient of a corresponding Jacquet module of $\Psi$, by the transitivity of Jacquet modules, there exists an irreducible subquotient $\tau \otimes \sigma$ of $s_{\left(p\left(a-a_{-}+2\right) / 2\right)}(\Psi)$, such that $t$ is a subquotient of a corresponding standard Jacquet module of $\tau \otimes \sigma$. Now we shall examine (10-33) to find all such $\tau \otimes \sigma$. We shall analyze which indexes and terms in the products and sums we can take to get such $\tau \otimes \sigma$ for a subquotient.

Note that the support of $\tau$ is $\left\{\nu^{(b-1) / 2}, \nu^{(a-1) / 2} \rho, \nu^{(a-1) / 2-1} \rho, \ldots, \nu^{\left(a_{-}-1\right) / 2+1} \rho\right\}$ (in general, the support is a multiset, but here it is actually a set; therefore we are in the regular situation).

First, to get $\tau \otimes \sigma$, we must not take $\nu^{-(b-1) / 2} \rho \otimes 1$ and $\nu^{-i} \rho \otimes 1$, since $\nu^{-(b-1) / 2} \rho$ and $\nu^{-i} \rho$ are not in the support of $\tau$.

Suppose that we get $\tau \otimes \sigma$ from some term where we take $1 \otimes \nu^{i} \rho$ in the right-hand side of (10-33). Then to get it in the support of $\tau$, it cannot come from $\nu^{(b-1) / 2} \rho \otimes 1$ and it cannot come from indexes $i^{\prime}, j^{\prime}$ since $i>\left(a_{-}-1\right) / 2$. It also cannot come from indexes $i^{\prime \prime}, j^{\prime \prime}$, since then we would also have $\left(b_{-}-1\right) / 2$ in the support of $\tau$, which is not the case. From this we conclude that there exist irreducible $\tau^{\prime} \otimes \sigma^{\prime} \leq \mu^{*}\left(\pi^{\prime}\right)$, such that the support of $\tau^{\prime}$ is non-empty and contained in the support of $\tau$. This implies (using Lemma 3.6) that either $b \in \operatorname{Jord}_{\rho}\left(\pi^{\prime}\right)$ or $2 l+1 \in \operatorname{Jord}_{\rho}\left(\pi^{\prime}\right)$ for some $\left(a_{-}-1\right) / 2+1 \leq l \leq(a-1) / 2$. But (10-23) and (9-1) imply that this is impossible.

Further, we must not take $1 \otimes \nu^{(b-1) / 2} \rho$. Otherwise, since $(b-1) / 2$ is greater than each of $(a-1) / 2,\left(a_{-}-1\right) / 2,\left(b_{-}-1\right) / 2$, to get $\nu^{(b-1) / 2} \rho$ in the support of $\tau$, we must have some $\tau^{\prime} \otimes \sigma^{\prime}$ with the same properties as in the above paragraph. We have seen that this implies the contradiction.

Therefore, we have proved that $\tau$ must be subquotient of

$$
\nu^{(b-1) / 2} \times \nu^{(a-1) / 2} \rho \times \nu^{(a-1) / 2-1} \rho \times \cdots \times \nu^{\left(a_{-}-1\right) / 2+1} \rho,
$$

multiplied by other terms. But since $\tau \otimes \sigma$ is a subquotient of $s_{\left(p\left(a-a_{-}+2\right) / 2\right)}(\Psi)$, we see that all other terms are equal to 1 . Thus, $\tau$ is a subquotient of (10-34). This implies that $\sigma$ must be a subquotient of

$$
\delta\left(\left[\nu^{-\left(b_{-}-1\right) / 2} \rho, \nu^{\left(b_{-}-1\right) / 2} \rho\right]\right) \times \delta\left(\left[\nu^{-\left(a_{-}-1\right) / 2} \rho, \nu^{\left(a_{-}-1\right) / 2} \rho\right]\right) \rtimes \pi^{\prime} .
$$


Finally, note that the multiplicity of $\nu^{(b-1) / 2} \otimes \nu^{(a-1) / 2} \rho \otimes \nu^{(a-1) / 2-1} \rho \otimes \cdots \otimes$ $\nu^{\left(a_{-}-1\right) / 2+1} \rho$ in a corresponding (standard) Jacquet module of (10-34) is one. On the other hand, (ii) of Lemma 4.3 implies that the multiplicity of

$$
\delta\left(\left[\nu^{-\left(b_{-}-1\right) / 2} \rho, \nu^{\left(b_{-}-1\right) / 2} \rho\right]\right) \otimes \delta\left(\left[\nu^{-\left(a_{-}-1\right) / 2} \rho, \nu^{\left(a_{-}-1\right) / 2} \rho\right]\right) \otimes \pi^{\prime}
$$

in a corresponding Jacquet module of (10-35) is four. Therefore the multiplicity of $t$ in a corresponding standard Jacquet module of $\Psi$ is $1 \cdot 4=4$. This ends the proof.

Since (10-30) embeds into (10-26), and since we have just shown that both representations have exactly four irreducible subrepresentations, (10-24) - (10-26) imply that $\pi_{\eta}$ embeds into (10-30). This and (1-2) imply

$$
\begin{aligned}
s_{G L}\left(\pi_{\eta}\right) \leq\left(\nu^{(b-1) / 2}+\nu^{-(b-1) / 2}\right) & \\
& \times s_{G L}\left(\bigoplus_{\eta^{\prime} \in\{ \pm 1\}} \delta\left(\left[\nu^{-\left(b_{-}-1\right) / 2} \rho, \nu^{\left(b_{-}-1\right) / 2} \rho\right]\right) \times \pi_{\eta^{\prime}}^{\prime}\right) .
\end{aligned}
$$

From (10-21), (10-7), $b>a+2$ (and (1-2)), we get that $\nu^{-(b-1) / 2} \rho$ is not a factor of $\pi_{\eta}$. Thus, (10-36) implies

$$
s_{G L}\left(\pi_{\eta}\right) \leq \nu^{(b-1) / 2} \times s_{G L}\left(\bigoplus_{\eta^{\prime} \in\{ \pm 1\}} \delta\left(\left[\nu^{-\left(b_{-}-1\right) / 2} \rho, \nu^{\left(b_{-}-1\right) / 2} \rho\right]\right) \times \pi_{\eta^{\prime}}^{\prime}\right) .
$$

Now Lemma 3.5 implies that $\pi_{\eta}$ is tempered (note that we did not use in the proof of Lemma 3.5 that $\tau$ is irreducible). Lemma 8.1 implies now that $\pi_{\eta}$ is square integrable. This ends the proof of the square integrability in the case $b-2 \in$ $\operatorname{Jord}_{\rho}(\pi)$ (and $\left.a<b\right)$.

10.2.2. It remains to consider the case $b-2 \notin \operatorname{Jor}_{\rho}(\pi)$ (we continue to assume $a<b)$. First we have a general

Lemma. If $b-2 \notin \operatorname{Jord}_{\rho}(\pi)$, then there exists an irreducible square integrable representation $\pi^{\prime \prime}$ such that

$$
\pi \hookrightarrow \nu^{(b-1) / 2} \rho \rtimes \pi^{\prime \prime}
$$

and (then)

$$
\operatorname{Jord}\left(\pi^{\prime \prime}\right)=(\operatorname{Jord}(\pi) \backslash\{(\rho, b)\}) \cup\{(\rho, b-2)\} .
$$

Remark. The proof of the lemma holds if one takes any $a^{\prime} \in \operatorname{Jord}_{\rho}(\pi)$ instead of $b$, which satisfies

$$
a^{\prime}>2 \quad \text { and } \quad a^{\prime}-2 \notin \operatorname{Jord}_{\rho}(\pi) .
$$

Note that $b$ satisfies the above condition.

Proof. We prove the lemma, and more generally the remark, by induction (for our fixed $\pi$, assuming that our general inductive assumption holds). We need to show the existence of an irreducible square integrable representation $\pi^{\prime \prime}$ such that

$$
\begin{gathered}
\pi \hookrightarrow \nu^{\left(a^{\prime}-1\right) / 2} \rho \rtimes \pi^{\prime \prime}, \\
\operatorname{Jord}\left(\pi^{\prime \prime}\right)=\left(\operatorname{Jord}(\pi) \backslash\left\{\left(\rho, a^{\prime}\right)\right\}\right) \cup\left\{\left(\rho, a^{\prime}-2\right)\right\} .
\end{gathered}
$$


Since (10-38') and Proposition 2.1 imply $\left(10-39^{\prime}\right)$, for the proof is enough to show the existence of the embedding $\left(10-38^{\prime}\right)$.

We discuss several possibilities.

First suppose that $\pi$ is strongly positive. Now we shall repeat a part of the construction in the seventh section, using the notation that we were using there. First, $\pi$ embeds into some representation $\sigma_{0}$ defined in (7-10). Let $a^{\prime}=a_{i}$ (then $i=1$ and $a_{i} \geq 3$, or $i>1$ and $\left.a_{i-1} \leq a_{i}-4\right)$. Denote by $\sigma_{0}^{\prime}$ the representation that one gets by substituting $\nu^{\left(a_{i}-1\right) / 2} \rho \times \delta\left(\left[\nu^{\left(\phi\left(a_{i}\right)+1\right) / 2} \rho, \nu^{\left(a_{i}-1\right) / 2-1} \rho\right]\right)$ instead of $\delta\left(\left[\nu^{\left(\phi\left(a_{i}\right)+1\right) / 2} \rho, \nu^{\left(a_{i}-1\right) / 2} \rho\right]\right)$ in (7-10) (all other terms remaining the same, as well as the order of the multiplication). Let $\sigma_{0}^{\prime \prime}$ be the representation that one obtains from $\sigma_{0}^{\prime}$, dropping $\nu^{\left(a_{i}-1\right) / 2} \rho$ from the definition of $\sigma_{0}^{\prime}$. Now $a^{\prime}-2 \notin \operatorname{Jord}_{\rho}(\pi)$ implies

$$
\sigma_{0}^{\prime} \cong \nu^{\left(a_{i}-1\right) / 2} \rho \rtimes \sigma_{0}^{\prime \prime} .
$$

Further, by the seventh section, $\sigma_{0}^{\prime \prime}$ has an irreducible square integrable subrepresentation, which we shall denote by $\pi^{\prime \prime}$ (for this we again use $a^{\prime}-2 \notin \operatorname{Jord}_{\rho}(\pi)$ ). Since $\sigma_{0}^{\prime}$ has a unique irreducible subrepresentation by Lemma 4.1, then $\pi \hookrightarrow \sigma_{0} \hookrightarrow \sigma_{0}^{\prime}$ and $\nu^{\left(a_{i}-1\right) / 2} \rho \rtimes \pi^{\prime \prime} \hookrightarrow \nu^{\left(a_{i}-1\right) / 2} \rho \rtimes \sigma_{0}^{\prime \prime} \cong \sigma_{0}^{\prime}$ imply $\left(10-38^{\prime}\right)$.

Suppose now that $\pi$ is not strongly positive. Then we can choose $c, c_{-} \in$ $\operatorname{Jord}_{\rho}(\pi)$ such that $\epsilon_{\pi}(c)=\epsilon_{\pi}\left(c_{-}\right)$and by Lemma 5.1 there exists an irreducible square integrable representation $\pi^{\prime \prime \prime}$ such that

$$
\pi \hookrightarrow \delta\left(\left[\nu^{-\left(c_{-}-1\right) / 2} \rho, \nu^{(c-1) / 2} \rho\right]\right) \rtimes \pi^{\prime \prime \prime} .
$$

Proposition 2.1 implies

$$
\operatorname{Jord}\left(\pi^{\prime \prime \prime}\right)=\operatorname{Jord}(\pi) \backslash\left\{(\rho, c),\left(\rho, c_{-}\right)\right\} .
$$

Note that

$$
\left[c_{-}, c\right] \cap \operatorname{Jord}_{\rho}\left(\pi^{\prime \prime \prime}\right)=\emptyset .
$$

We shall consider three cases.

First consider the case $a^{\prime}=c$. Now $a^{\prime}=c \geq c_{-}+4$. This and (10-40) imply

$$
\begin{aligned}
& \pi \hookrightarrow \delta\left(\left[\nu^{-\left(c_{-}-1\right) / 2} \rho, \nu^{(c-1) / 2} \rho\right]\right) \rtimes \pi^{\prime \prime \prime} \\
& \hookrightarrow \nu^{(c-1) / 2} \rho \times \delta\left(\left[\nu^{-\left(c_{-}-1\right) / 2} \rho, \nu^{(c-1) / 2-1} \rho\right]\right) \rtimes \pi^{\prime \prime \prime} \\
& \hookrightarrow \nu^{(c-1) / 2} \rho \times \delta\left(\left[\nu^{\left(c_{-}-1\right) / 2+1} \rho, \nu^{(c-1) / 2-1} \rho\right]\right) \\
& \quad \times \delta\left(\left[\nu^{-\left(c_{-}-1\right) / 2} \rho, \nu^{\left(c_{-}-1\right) / 2-1} \rho\right]\right) \rtimes \pi^{\prime \prime \prime} .
\end{aligned}
$$

Now $\delta\left(\left[\nu^{-\left(c_{-}-1\right) / 2} \rho, \nu^{(c-1) / 2-1} \rho\right]\right) \rtimes \pi^{\prime \prime \prime}$ has two irreducible subrepresentations, which are square integrable by our general inductive assumption (see (10-41)). Denote them by $\pi_{\eta^{\prime \prime}}^{\prime \prime}, \eta^{\prime} \in\{ \pm 1\}$. By Lemma 4.5 and Remark 4.2, (10-44) has at most two irreducible subrepresentations. Therefore (10-43) has at most two irreducible subrepresentations. This implies $\pi \hookrightarrow \nu^{(c-1) / 2} \rho \rtimes \pi_{\eta^{\prime}}^{\prime \prime \prime}$ for some $\eta^{\prime}$. This proves $\left(10-38^{\prime}\right)$.

Now let $a^{\prime}=c_{-}$. Then

$$
\begin{aligned}
& \pi \hookrightarrow \delta\left(\left[\nu^{-\left(c_{-}-1\right) / 2} \rho, \nu^{(c-1) / 2} \rho\right]\right) \rtimes \pi^{\prime \prime \prime} \\
& \hookrightarrow \delta\left(\left[\nu^{-\left(c_{-}-1\right) / 2+1} \rho, \nu^{(c-1) / 2} \rho\right]\right) \times \nu^{-\left(c_{-}-1\right) / 2} \rho \rtimes \pi^{\prime \prime \prime} \\
& \quad \cong \delta\left(\left[\nu^{-\left(c_{-}-1\right) / 2+1} \rho, \nu^{(c-1) / 2} \rho\right]\right) \times \nu^{\left(c_{-}-1\right) / 2} \rho \rtimes \pi^{\prime \prime \prime} .
\end{aligned}
$$


The last equivalence follows from Lemma 5.4 .1 of $\left[\mathrm{M} 2\right.$ (since $\left(c_{-}-1\right) / 2=(d+1) / 2$ for some $d \in \operatorname{Jord}_{\rho}(\pi)$ would imply $d=c_{-}-2=a^{\prime}-2 \in \operatorname{Jord}_{\rho}(\pi)$, which contradicts our assumptions). From the above embeddings, we get

$$
\begin{aligned}
\pi \hookrightarrow \nu^{\left(c_{-}-1\right) / 2} \rho \times \delta\left(\left[\nu^{-\left(c_{-}-1\right) / 2+1} \rho, \nu^{(c-1) / 2} \rho\right]\right) \rtimes \pi^{\prime \prime \prime} \\
=\nu^{\left(c_{-}-1\right) / 2} \rho \times \delta\left(\left[\nu^{-\left(c_{-}-2-1\right) / 2} \rho, \nu^{(c-1) / 2} \rho\right]\right) \rtimes \pi^{\prime \prime \prime} .
\end{aligned}
$$

Note that

$$
\left[c_{-}-2, c\right] \cap \operatorname{Jord}_{\rho}\left(\pi^{\prime \prime \prime}\right)=\emptyset
$$

(since $a^{\prime}-2=c_{-}-2 \notin \operatorname{Jord}_{\rho}(\pi)$ ). Therefore

$$
\delta\left(\left[\nu^{-\left(c_{-}-2-1\right) / 2} \rho, \nu^{(c-1) / 2} \rho\right]\right) \rtimes \pi^{\prime \prime \prime}
$$

has exactly two irreducible subrepresentations. Denote them by $\pi_{\eta^{\prime}}^{\prime \prime \prime}, \eta^{\prime} \in\{ \pm 1\}$. They are square integrable by the general inductive assumption. Further

$$
\begin{aligned}
& \pi \hookrightarrow \nu^{\left(c_{-}-1\right) / 2} \rho \times \delta\left(\left[\nu^{-\left(c_{-}-1\right) / 2+1} \rho, \nu^{(c-1) / 2} \rho\right]\right) \rtimes \pi^{\prime \prime \prime} \\
\hookrightarrow & \nu^{\left(c_{-}-1\right) / 2} \rho \times \delta\left(\left[\nu^{\left(c_{-}-1\right) / 2} \rho, \nu^{(c-1) / 2} \rho\right]\right) \times \delta\left(\left[\nu^{-\left(c_{-}-1\right) / 2+1} \rho, \nu^{\left(c_{-}-1\right) / 2-1} \rho\right]\right) \rtimes \pi^{\prime \prime \prime} .
\end{aligned}
$$

Using $\left[c_{-}, c\right] \cap \operatorname{Jord}_{\rho}\left(\pi^{\prime \prime \prime}\right)=\emptyset$, in a similar way as in the proof of Lemma 4.5, one gets that the last representation has at most two irreducible subrepresentations (the only difference from the proof of Lemma 4.5 is that one does not use regularity, but instead uses the well-known fact that $\nu^{\left(c_{-}-1\right) / 2} \rho \times \delta\left(\left[\nu^{\left(c_{-}-1\right) / 2} \rho, \nu^{(c-1) / 2} \rho\right]\right)$ has multiplicity one in $\nu^{\left(c_{-}-1\right) / 2} \rho \times \prod_{i=0}^{\left(c-c_{-}\right) / 2} \nu^{\left(c_{-}-1\right) / 2+i} \rho$. From this and the above embeddings we conclude that

$$
\pi \hookrightarrow \nu^{\left(c_{-}-1\right) / 2} \rho \rtimes \pi_{\eta^{\prime}}^{\prime \prime \prime}
$$

for some $\eta^{\prime} \in\{ \pm 1\}$. This shows (10-38') in the case $a^{\prime}=c_{-}$.

Suppose finally that $a^{\prime} \notin\left\{c_{-}, c\right\}$. Then $a^{\prime} \notin\left[c_{-}, c\right]$. Further note that

$$
\nu^{\left(a^{\prime}-1\right) / 2} \rho \times \delta\left(\left[\nu^{-\left(c_{-}-1\right) / 2} \rho, \nu^{(c-1) / 2} \rho\right]\right) \cong \delta\left(\left[\nu^{-\left(c_{-}-1\right) / 2} \rho, \nu^{(c-1) / 2} \rho\right]\right) \times \nu^{\left(a^{\prime}-1\right) / 2} \rho
$$

since either $a^{\prime}<c_{-}$or $(c-1) / 2+1<\left(a^{\prime}-1\right) / 2$ by our assumptions. Then $c \leq a^{\prime}-4$. Further, $a^{\prime} \in \operatorname{Jord}_{\rho}\left(\pi^{\prime \prime \prime}\right)$ by (10-41) and $a^{\prime}-2 \notin \operatorname{Jord}_{\rho}\left(\pi^{\prime \prime \prime}\right)$. Therefore, we can apply the inductive assumption. Applying it, we get that there exists a square integrable representation $\pi^{\prime \prime \prime \prime}$ such that

$$
\begin{aligned}
\operatorname{Jord}\left(\pi^{\prime \prime \prime \prime \prime}\right)=\left(\operatorname{Jord}\left(\pi^{\prime \prime \prime}\right) \backslash\left\{\left(\rho, a^{\prime}\right)\right\}\right) \cup\left\{\left(\rho, a^{\prime}-2\right)\right\} \\
\quad=\left(\operatorname{Jord}(\pi) \backslash\left\{(\rho, c),\left(\rho, c_{-}\right),\left(\rho, a^{\prime}\right)\right\}\right) \cup\left\{\left(\rho, a^{\prime}-2\right)\right\} .
\end{aligned}
$$

Now

$$
\begin{aligned}
& \pi \hookrightarrow \delta\left(\left[\nu^{-\left(c_{-}-1\right) / 2} \rho, \nu^{(c-1) / 2} \rho\right]\right) \rtimes \pi^{\prime \prime \prime} \\
& \hookrightarrow \delta\left(\left[\nu^{-\left(c_{-}-1\right) / 2} \rho, \nu^{(c-1) / 2} \rho\right]\right) \times \nu^{\left(a^{\prime}-1\right) / 2} \rho \rtimes \pi^{\prime \prime \prime \prime} \\
& \cong \nu^{\left(a^{\prime}-1\right) / 2} \rho \times \delta\left(\left[\nu^{-\left(c_{-}-1\right) / 2} \rho, \nu^{(c-1) / 2} \rho\right]\right) \rtimes \pi^{\prime \prime \prime \prime} \\
& \hookrightarrow \nu^{\left(a^{\prime}-1\right) / 2} \rho \times \delta\left(\left[\nu^{\left(c_{-}-1\right) / 2+1} \rho, \nu^{(c-1) / 2} \rho\right]\right) \\
& \\
& \times \delta\left(\left[\nu^{-\left(c_{-}-1\right) / 2} \rho, \nu^{\left(c_{-}-1\right) / 2} \rho\right]\right) \rtimes \pi^{\prime \prime \prime \prime} .
\end{aligned}
$$


Now (10-46) implies that $\delta\left(\left[\nu^{-\left(c_{-}-1\right) / 2} \rho, \nu^{(c-1) / 2} \rho\right]\right) \rtimes \pi^{\prime \prime \prime \prime \prime}$ has exactly two irreducible subrepresentations, and they are square integrable by the general inductive assumption. Denote them by $\pi_{\eta^{\prime}}^{\prime \prime \prime}, \eta^{\prime} \in\{ \pm\}$. Lemma 4.5, Remark 4.2 and (4-46) imply that (10-50) has at most two irreducible subrepresentations. Then, the same holds for (10-49). This implies that $\pi \hookrightarrow \nu^{\left(a^{\prime}-1\right) / 2} \rho \times \pi_{\eta^{\prime}}^{\prime \prime \prime}$ for some $\eta^{\prime}$. This proves $\left(10-38^{\prime}\right)$. Now the proof of the lemma and the remark is complete.

Using this lemma, we get

$$
\begin{aligned}
\pi_{\eta} \hookrightarrow \delta\left(\left[\nu^{-\left(a_{-}-1\right) / 2} \rho, \nu^{(a-1) / 2} \rho\right]\right) \rtimes \pi \\
\hookrightarrow \delta\left(\left[\nu^{-\left(a_{-}-1\right) / 2} \rho, \nu^{(a-1) / 2} \rho\right]\right) \times \nu^{(b-1) / 2} \rho \rtimes \pi^{\prime \prime} .
\end{aligned}
$$

Recall $a<b$. We shall consider separately the cases of $a+2<b$ and $a+2=b$.

10.2.2.1. First we shall consider the case

$$
a+2<b
$$

Since $(a-1) / 2+1<(b-1) / 2,(10-51)$ implies

$$
\begin{aligned}
& (10-52) \quad \pi_{\eta} \hookrightarrow \nu^{(b-1) / 2} \rho \times \delta\left(\left[\nu^{-\left(a_{-}-1\right) / 2} \rho, \nu^{(a-1) / 2} \rho\right]\right) \rtimes \pi^{\prime \prime} \\
& \hookrightarrow \nu^{(b-1) / 2} \rho \times \delta\left(\left[\nu^{\left(a_{-}-1\right) / 2+1} \rho, \nu^{(a-1) / 2} \rho\right]\right) \times \delta\left(\left[\nu^{-\left(a_{-}-1\right) / 2} \rho, \nu^{\left(a_{-}-1\right) / 2} \rho\right]\right) \rtimes \pi^{\prime \prime} .
\end{aligned}
$$

Now (10-39) implies that $\delta\left(\left[\nu^{-\left(a_{-}-1\right) / 2} \rho, \nu^{(a-1) / 2} \rho\right]\right) \rtimes \pi^{\prime \prime}$ has exactly two irreducible subrepresentations. They are square integrable by the inductive assumption. Denote them by $\pi_{\eta^{\prime}}^{\prime \prime}, \eta^{\prime}\{ \pm 1\}$. Further, (10-39), Lemma 4.5 and Remark 4.2 imply that the representation in the second row of (10-52) has at most two irreducible subrepresentations. This implies

$$
\pi_{\eta} \hookrightarrow \nu^{(b-1) / 2} \rho \rtimes \pi_{\eta^{\prime}}^{\prime \prime}
$$

for some $\eta^{\prime}$. Note that $\pi_{\eta} \hookrightarrow \delta\left(\left[\nu^{-\left(a_{-}-1\right) / 2} \rho, \nu^{(a-1) / 2} \rho\right]\right) \rtimes \pi$ and Lemma 5.2 applied to $\pi$ imply that $\nu^{(b-1) / 2} \rho$ is not a factor of $\pi_{\eta}$ (similarly as in 10.2.1; see (10-21)). Now (1-2) and (10-53) imply $s_{G L}\left(\pi_{\eta}\right) \leq \nu^{(b-1) / 2} \rho \times s_{G L}\left(\pi_{\eta^{\prime}}^{\prime \prime}\right)$. Now Lemma 3.5 implies that $\pi_{\eta}$ is square integrable.

Therefore, we have settled the case $a+2<b$ (recall that we assumed $b-2 \notin$ $\left.\operatorname{Jord}_{\rho}(\pi)\right)$. Finally, it remains to consider only the case $b=a+2$.

\section{END OF PROOF OF SQUARE INTEGRABILITY}

We continue with the notation of the last section, and assume additionally that

$$
a+2=b \text {. }
$$

This is the only case where we have not yet proved the square integrability of $\pi_{\eta}$.

We shall suppose now that $\pi_{\eta}$ is not square integrable.

If $b_{-} \in \operatorname{Jord}_{\rho}(\pi)$ is not defined, then the definition of $b$ implies that $\pi$ is strongly positive. Suppose that $b_{-} \in \operatorname{Jord}_{\rho}(\pi)$ is defined. Then $a+2=b$ and (9-1) imply $b_{-} \leq a_{-}-2$. Now Lemma 3.6 implies that $\nu^{-\left(b_{-}-1\right) / 2-1}, \nu^{-\left(b_{-}-1\right) / 2-2}, \ldots$ are not factors of $\pi$. Note that $-\left(a_{-}-1\right) / 2 \leq-\left(b_{-}-1\right) / 2-1$. Therefore, we have

$$
\pi \text { has no factors in the set }\left\{\nu^{-\left(a_{-}-1\right) / 2-z} \rho ; z \in \mathbb{Z}_{+}\right\},
$$

regardless if $b_{-}$is defined or not. Recall further

$$
\pi_{\eta} \hookrightarrow \delta\left(\left[\nu^{-\left(a_{-}-1\right) / 2} \rho, \nu^{(a-1) / 2} \rho\right]\right) \rtimes \pi .
$$


This, (1-2), (1-4) and (11-1) imply that

$$
\pi_{\eta} \text { has no factors in the set }\left\{\nu^{-(a-1) / 2-1-z} \rho ; z \in \mathbb{Z}_{+}\right\} \text {. }
$$

Suppose that $\nu^{-(a-1) / 2}$ is not a factor of $\pi_{\eta}$. Then from (11-2) it follows that

$$
\pi_{\eta} \hookrightarrow \nu^{(a-1) / 2} \rho \times \delta\left(\left[\nu^{-\left(a_{-}-1\right) / 2} \rho, \nu^{(a-1) / 2-1} \rho\right]\right) \rtimes \pi .
$$

Using the inductive assumption, from this we get in the same way as before (for example in 10.1) that $\pi_{\eta}$ is square integrable.

Therefore, $\nu^{-(a-1) / 2}$ is a factor of $\pi_{\eta}$.

Recall that Lemma 10.2 implies

$$
\pi \hookrightarrow \nu^{(b-1) / 2} \rho \rtimes \pi^{\prime \prime},
$$

where

$$
\operatorname{Jord}_{\rho}\left(\pi^{\prime \prime}\right)=\left(\operatorname{Jord}_{\rho}(\pi) \backslash\{b\}\right) \cup\{a\} .
$$

We need to keep in mind all the time that $b=a+2$, which implies $(b-1) / 2=$ $(a-1) / 2+1$. Now

$$
\begin{aligned}
\pi_{\eta} \hookrightarrow \delta\left(\left[\nu^{-\left(a_{-}-1\right) / 2} \rho, \nu^{(a-1) / 2} \rho\right]\right) \rtimes \pi \\
\hookrightarrow \delta\left(\left[\nu^{-\left(a_{-}-1\right) / 2} \rho, \nu^{(a-1) / 2} \rho\right]\right) \rtimes \nu^{(b-1) / 2} \rho \rtimes \pi^{\prime \prime} .
\end{aligned}
$$

Now we two technical lemmas. The first one is very simple.

11.1. Lemma. If $\nu^{z} \rho \otimes \sigma \leq \mu^{*}(\pi)$ for some irreducible representation $\sigma$ and $z \in$ $(1 / 2) \mathbb{Z}$, then $z \leq(b-1) / 2$.

Proof. Suppose $z>(b-1) / 2$. This implies $2 z+1>b$. Lemma 3.6 implies $2 z+1 \in \operatorname{Jord}_{\rho}(\pi)$. Lemma 3.1 and Lemma 3.2 imply the existence of an irreducible representation $\sigma^{\prime}$, such that $\pi \hookrightarrow \nu^{2 z+1} \rho \rtimes \sigma^{\prime}$. By the definition of $b, 2 z-1 \in$ $\operatorname{Jord}_{\rho}(\pi)$ (since $2 z+1>b$ ). Now the definition of the partial function $\epsilon$ implies $\epsilon(2 z-1,2 z+1)=1$. This contradicts the definition of $b$ (since $2 z+1>b$ ).

11.2. Lemma. (i) If there exists an embedding

$$
\pi_{\eta} \hookrightarrow \delta\left(\left[\nu^{-(a-1) / 2}, \nu^{l}\right]\right) \rtimes \sigma^{\prime},
$$

with $\sigma^{\prime}$ irreducible and $l \in\left\{-(a-1) / 2+z ; z \in \mathbb{Z}_{+}\right\}$, then $l=(b-1) / 2$.

(ii) There exists an embedding

$$
\pi_{\eta} \hookrightarrow \delta\left(\left[\nu^{-(a-1) / 2}, \nu^{(b-1) / 2}\right]\right) \rtimes \sigma^{\prime},
$$

with irreducible $\sigma^{\prime}$.

(iii) Any representation $\sigma^{\prime}$ which satisfies (ii), must be square integrable. Further,

$$
\operatorname{Jord}_{\rho}\left(\sigma^{\prime}\right)=\left(\operatorname{Jord}_{\rho}(\pi) \backslash\{b\}\right) \cup\left\{a_{-}\right\} .
$$

Proof. The proof of (i) and (ii) proceeds in a similar way as the proof of Lemma 10.1 .

Since $\nu^{-(a-1) / 2-1} \rho$ is not a factor of $\pi_{\eta}$, Lemma 3.4 implies

$$
\pi_{\eta} \hookrightarrow \delta\left(\left[\nu^{-(a-1) / 2} \rho, \nu^{\left(a^{\prime}-1\right) / 2} \rho\right]\right) \rtimes \sigma,
$$

for some irreducible $\sigma$ and $a^{\prime} \in \mathbb{Z}$ such that $a+a^{\prime} \in 2 \mathbb{Z}$ and $\left(a^{\prime}-1\right) / 2-(-(a-1) / 2) \geq$ 0 . Frobenius reciprocity implies that

$$
\nu^{\left(a^{\prime}-1\right) / 2} \rho \otimes \nu^{\left(a^{\prime}-1\right) / 2-1} \rho \otimes \cdots \otimes \nu^{-(a-1) / 2} \rho \otimes \rho_{1} \otimes \rho_{2} \otimes \cdots \otimes \rho_{l} \otimes \pi_{\text {cusp }}
$$


is an irreducible subquotient of a corresponding Jacquet module of $\pi_{\eta}$ ( $\rho_{i}$ are irreducible cuspidal representations). Now (11-60) must be a subquotient of a corresponding Jacquet module of some irreducible subquotient $\tau \otimes \sigma$ of $s_{\left(\left(a+a_{-}\right) / 2\right)}\left(\pi_{\eta}\right)$.

Suppose first $a^{\prime} \leq a_{-}$. This implies that $\nu^{-(a-1) / 2} \rho$ must be in the support of $\tau$. Clearly, $\tau \otimes \sigma \leq s_{\left(\left(a+a_{-}\right) / 2\right)}\left(\delta\left(\left[\nu^{-\left(a_{-}-1\right) / 2} \rho, \nu^{(a-1) / 2} \rho\right]\right) \rtimes \pi\right)$. Now (10-9) and (11-1) imply $\sigma \otimes \tau=\delta\left(\left[\nu^{-(a-1) / 2} \rho, \nu^{\left(a_{-}-1\right) / 2} \rho\right]\right) \otimes \pi$. Lemma 4.4 implies that $\pi_{\eta}$ is a Langlands quotient of $\delta\left(\left[\nu^{-\left(a_{-}-1\right) / 2} \rho, \nu^{(a-1) / 2} \rho\right]\right) \rtimes \pi$, which contradicts the reducibility of $\delta\left(\left[\nu^{\left(a_{-}-1\right) / 2} \rho, \nu^{(a-1) / 2} \rho\right]\right) \rtimes \pi$.

Suppose now $a_{-}<a^{\prime}$. Since (11-10) is a subquotient of a Jacquet module of $\pi_{\eta}$, Lemma 3.1 and Lemma 3.2 imply that $\nu^{\left(a^{\prime}-1\right) / 2} \rho \otimes \gamma \leq s_{(p)}\left(\pi_{\eta}\right)$, for some irreducible representation $\gamma$, such that $\nu^{-(a-1) / 2} \rho$ is a factor of $\gamma$. Now (11-2) implies

$$
\nu^{\left(a^{\prime}-1\right) / 2} \rho \otimes \gamma \leq s_{(p)}\left(\delta\left(\left[\nu^{-\left(a_{-}-1\right) / 2} \rho, \nu^{(a-1) / 2} \rho\right]\right) \rtimes \pi\right) .
$$

Write $s_{(p)}(\pi)=\sum_{i} \mu_{i} \otimes \lambda_{i}$ as a sum of irreducible representations (as we did in (10-11)). In 10.1 we have computed the formula for

$$
s_{(p)}\left(\delta\left(\left[\nu^{-\left(a_{-}-1\right) / 2} \rho, \nu^{(a-1) / 2} \rho\right]\right) \rtimes \pi\right)
$$

(the first displayed formula after (10-11)). We shall use this formula now. The present assumption $a_{-}<a^{\prime}$ implies that $\nu^{\left(a^{\prime}-1\right) / 2} \rho \otimes \gamma$ cannot be a subquotient of (10-13). Further (1-2), (1-4) and (11-1) imply that $\nu^{-(a-1) / 2} \rho$ is not a factor of the representation $\delta\left(\left[\nu^{-\left(a_{-}-1\right) / 2} \rho, \nu^{(a-1) / 2-1} \rho\right]\right) \rtimes \pi$. This implies that $\nu^{\left(a^{\prime}-1\right) / 2} \rho \otimes \gamma$ is not a subquotient of (10-12). Therefore, $\nu^{\left(a^{\prime}-1\right) / 2} \rho \cong \mu_{i}$ for some $i$. Lemma 3.6 now implies $a^{\prime} \in \operatorname{Jord}_{\rho}(\pi)$. Since $a_{-}<a^{\prime}$, (9-1) implies $a<a^{\prime}$. Now (9-1) implies $b \leq a^{\prime}$.

Suppose $b<a^{\prime}$. Then, to get $\delta\left(\left[\nu^{-(a-1) / 2} \rho, \nu^{\left(a^{\prime}-1\right) / 2-1} \rho\right]\right) \otimes \sigma^{\prime}$ as a subquotient of (10-9) we first need to take $i=(a-1) / 2$ in $(10-9)$ (since $\nu^{-(a-1) / 2} \rho$ is not a factor of $\pi)$. Therefore, we must have $\delta\left(\left[\nu^{\left(a_{-}-1\right) / 2+1} \rho, \nu^{\left(a^{\prime}-1\right) / 2-1} \rho\right]\right) \otimes \sigma^{\prime \prime} \leq \mu^{*}(\pi)$ for some irreducible representation $\sigma^{\prime \prime}$. This contradicts the above lemma (since we would have $\nu^{\left(a^{\prime}-1\right) / 2-1} \rho \otimes \sigma^{\prime \prime \prime} \leq \mu^{*}(\pi)$ for some irreducible representation $\sigma^{\prime \prime \prime}$, with $\left.\left(a^{\prime}-1\right) / 2>(b-1) / 2\right)$. This proves (i).

Now (ii) follows from (i) and (i) of Lemma 3.4.

It remains to prove (iii). From 10.2.2, we know that there exists an irreducible square integrable representation $\pi^{\prime \prime}$ and an embedding:

$$
\pi \hookrightarrow \nu^{(b-1) / 2} \rho \times \pi^{\prime \prime} .
$$

Moreover we know by Proposition 2.1 that

$$
\operatorname{Jord}_{\rho}\left(\pi^{\prime \prime}\right)=\left(\operatorname{Jord}_{\rho}(\pi) \backslash\{b\}\right) \cup\{b-2\} .
$$

Further, since $a=b-2 \in \operatorname{Jord}_{\rho}\left(\pi^{\prime \prime}\right)$ and $(a-2) \notin \operatorname{Jord}_{\rho}\left(\pi^{\prime \prime}\right)$, we can again use Remark 10.2.2. Continuing to use this remark several times (in the cases when we can), we shall get

$$
\pi \hookrightarrow \nu^{\left(a_{-}-1\right) / 2+1} \rho \times \cdots \times \nu^{(a-1) / 2} \rho \times \nu^{(b-1) / 2} \rho \rtimes \pi^{\prime},
$$

where

$$
\operatorname{Jord}_{\rho}\left(\pi^{\prime}\right)=\left(\operatorname{Jord}_{\rho}(\pi) \backslash\{b\}\right) \cup\left\{a_{-}\right\} .
$$

In particular, if $a^{\prime} \in \operatorname{Jord}_{\rho}\left(\pi^{\prime}\right)$, then

$$
\left(a^{\prime}-1\right) / 2 \notin\left[(a+1) / 2,\left(a_{-}+1\right) / 2\right] .
$$


Now (11-11) and the definition of $\pi_{\eta}$ imply that we have the embedding

$$
\begin{aligned}
\pi_{\eta} \hookrightarrow \delta\left(\left[\nu^{-\left(a_{-}-1\right) / 2} \rho, \nu^{(a-1) / 2} \rho\right]\right) \\
\times \nu^{\left(a_{-}-1\right) / 2+1} \rho \times \cdots \times \nu^{(a-1) / 2} \rho \times \nu^{(a+1) / 2} \rho \rtimes \pi^{\prime} .
\end{aligned}
$$

By (ii), we have the embedding

$$
\pi_{\eta} \hookrightarrow \delta\left(\left[\nu^{-(a-1) / 2} \rho, \nu^{(a+1) / 2} \rho\right]\right) \times \sigma^{\prime} .
$$

Consider any such embedding. Frobenius reciprocity implies that

$$
\delta\left(\left[\nu^{-(a-1) / 2} \rho, \nu^{(a+1) / 2} \rho\right]\right) \otimes \sigma^{\prime}
$$

is a subquotient of a corresponding Jacquet module of

$$
\delta\left(\left[\nu^{-\left(a_{-}-1\right) / 2} \rho, \nu^{(a-1) / 2} \rho\right]\right) \times \nu^{\left(a_{-}-1\right) / 2+1} \rho \times \cdots \times \nu^{(a-1) / 2} \rho \times \nu^{(a+1) / 2} \rho \rtimes \pi^{\prime} .
$$

We shall show now that $\sigma^{\prime} \cong \pi^{\prime}$. This (and (11-12)) will imply (iii).

First we shall write $\mu^{*}$ of $(11-17)$ :

$$
\begin{aligned}
&\left(\prod_{k=(a--1) / 2+1}^{(a+1) / 2}\left(\nu^{k} \rho \otimes 1+\nu^{-k} \rho \otimes 1+1 \otimes \nu^{k} \rho\right)\right) \\
& \times\left(\sum_{i=-\left(a_{-}-1\right) / 2-1}^{(a-1) / 2} \sum_{j=i}^{(a-1) / 2} \delta\left(\left[\nu^{-i} \rho, \nu^{\left(a_{-}-1\right) / 2} \rho\right]\right) \times \delta\left(\left[\nu^{j+1} \rho, \nu^{(a-1) / 2} \rho\right]\right)\right. \\
&\left.\delta\left(\left[\nu^{i+1} \rho, \nu^{j} \rho\right]\right)\right) \rtimes \mu^{*}\left(\pi^{\prime}\right) .
\end{aligned}
$$

Now we shall analyze when we can get (11-16) for a subquotient of (11-18).

The first conclusion is that for $k=(a+1) / 2$ we need to take the term $\nu^{(a+1) / 2} \rho \otimes$ 1 , since $b \notin \operatorname{Jord}_{\rho}\left(\pi^{\prime}\right)$.

Now look at $k=(a-1) / 2$. Suppose that we have taken the term $1 \otimes \nu^{(a-1) / 2} \rho$. We consider two possibilities. Suppose $j+1 \leq(a-1) / 2$. Then $i \leq(a-1) / 2-1$. Therefore, in the term $\delta\left(\left[\nu^{-(a-1) / 2} \rho, \nu^{(a+1) / 2} \rho\right]\right)$ in $(11-16), \nu^{-(a-1) / 2} \rho$ must come from $\mu^{*}\left(\pi^{\prime}\right)$. This directly implies that $\pi^{\prime}$ is not square integrable. Thus, $j=$ $(a-1) / 2$. This would imply that $a \in \operatorname{Jord}_{\rho}\left(\pi^{\prime}\right)$. This cannot happen. Therefore, for $k=(a-1) / 2$ we must not take the term $1 \otimes \nu^{(a-1) / 2} \rho$ (if we want to get (11-16) for a subquotient). The two possibilities remain.

Suppose that we have taken the term $\nu^{-(a-1) / 2} \rho \otimes 1$. Then we need to take $-i \geq-(a-1) / 2+1$ in (11-18). Suppose $-i>-(a-1) / 2+1$. Since $a \notin \operatorname{Jord}_{\rho}\left(\pi^{\prime}\right)$, we get $j+1 \leq(a-1) / 2$. This implies that $\pi^{\prime}$ is not square integrable. Thus $-i=-(a-1) / 2+1$, i.e. $i=(a-1) / 2-1$. Now $j=(a-1) / 2-1$ or $(a-1) / 2$. If $j=(a-1) / 2$, then $a \in \operatorname{Jord}_{\rho}\left(\pi^{\prime}\right)$, which is impossible. Thus, $j=(a-1) / 2-1$. Now for the other $k$ 's (i.e. when $k<(a-1) / 2$ ), we must take terms $\nu^{k} \rho \otimes 1$. This implies $\sigma^{\prime} \cong \pi^{\prime}$.

The other possibility is that we have taken the term $\nu^{(a-1) / 2} \rho \otimes 1$. This implies $j=(a-1) / 2$. Suppose $-i>-(a-1) / 2$ (i.e. $i<(a-1) / 2)$. This easily implies that $\pi^{\prime}$ is not square integrable. Therefore, $i=(a-1) / 2$. This implies $j=(a-1) / 2$. Again for the remaining $k$ 's, one must take the terms $\nu^{k} \rho \otimes 1$. This again implies $\sigma^{\prime} \cong \pi^{\prime}$.

The proof of (iii) is now complete. 
Suppose $b=\max \left(\operatorname{Jord}_{\rho}\right)$. Now (iii) of the above lemma and the tenth section imply the contradiction. Thus, $b<\max \left(\operatorname{Jord}_{\rho}\right)$. Nevertheless, using the above lemma, in finitely many steps we come to the contradiction.

This contradiction implies that our assumption that $\pi_{\eta}$ is not square integrable cannot hold. Thus, $\pi_{\eta}$ is square integrable. This ends the proof of the square integrability.

Note that the representations $\pi_{\eta}$ are constructed recursively. There are the cases when one can define them more directly. There are examples of constructions of families of such representations in [T4] (and proofs of their square integrability, together with explicit estimates of their Jacquet modules and description of some other properties).

\section{JORDAN BLOCKS AND CUSPIDAL REDUCIBILITY}

In this section only, we shall not assume that (BA) holds (and also we shall not assume that (A) holds). For simplicity, we shall assume in this section that groups $S_{n}$ are split.

Let $\rho$ be an irreducible $F^{\prime} / F$-selfdual cuspidal representation of $G L(p)$ and let $\sigma$ be an irreducible cuspidal representation of $S_{q}$. We are interested in the reducibility points of the family $\nu^{\alpha} \rho \rtimes \sigma, \alpha \in \mathbb{R}$. First, $\nu^{\alpha} \rho \rtimes \sigma$ reduces for some $\alpha \in \mathbb{R}$. In all the known cases when the reducibility points of such families are computed, the following holds:

(HI)

if $\nu^{\alpha} \rho \rtimes \sigma$ reduces, then $\alpha \in(1 / 2) \mathbb{Z}$ and $\nu^{\beta} \rho \rtimes \sigma$ is irreducible for $\beta \in \mathbb{R} \backslash\{ \pm \alpha\}$.

This is expected to hold in general. The first general result in this direction is due to Shahidi ([Sh1]). Shahidi proved that (HI) holds if $\sigma$ is generic (he showed much more; see below). Assume that (HI) holds for $\rho$ and $\sigma$ (note that (BA) implies $(\mathrm{HI})$ ). Then the non-negative reducibility point is unique. We shall denote it by

$$
\alpha(\rho, \sigma) \text {. }
$$

Shahidi proved

$$
\text { if } \sigma \text { is generic, then } \alpha(\rho, \sigma) \in\{0, \pm 1 / 2, \pm 1\} \text {. }
$$

In particular, this implies, for the simplest case when $\sigma$ is the trivial representation 1 of the trivial group, that

$$
\alpha(\rho, 1) \in\{0, \pm 1 / 2, \pm 1\}
$$

In the lemma below, we shall see that (BA), which comes from a study of Arthur's conjectures, implies that the following holds:

$$
\begin{aligned}
& \text { if } \nu^{\alpha} \rho \rtimes \sigma \text { reduces, then } \alpha-\alpha(\rho, 1) \in \mathbb{Z} \\
& \quad \text { and } \nu^{\beta} \rho \rtimes \sigma \text { is irreducible for } \beta \in \mathbb{R} \backslash\{ \pm \alpha\} .
\end{aligned}
$$

Obviously, (D) implies (HI) (using (12-2)).

12.1. Lemma. The assumptions $(B A)$ and $(D)$ are equivalent.

Proof. Assume that (D) holds.

Suppose that $\rho \rtimes \sigma$ reduces. Then by (D) and (12-2), $\rho \rtimes 1$ or $\nu \rho \rtimes 1$ reduces. This implies (by Shahidi's work) that $L\left(\rho, R_{d_{\rho}}, s\right)$ has no pole at $s=0$ (see also (i) of Remark 2.3). Thus if $a \in \operatorname{Jord}_{\rho}(\sigma)$, then $a$ is odd. For any odd $a \in \mathbb{N}, \delta(\rho, a) \rtimes \sigma$ 
reduces by Proposition 4.4 of [T2]. Thus, $\operatorname{Jord}_{\rho}(\sigma)=\emptyset$. Therefore, (BA) holds for this pair.

Let $\nu^{1 / 2} \rho \rtimes \sigma$ be reducible. Then $\nu^{1 / 2} \rho \rtimes 1$ reduces by (D). This implies that $L\left(\rho, R_{d_{\rho}}, s\right)$ has a pole at $s=0$. Thus, each $a \in \operatorname{Jord}_{\rho}(\sigma)$ must be even. Now Proposition 4.3 of T2 implies $\operatorname{Jord}_{\rho}(\sigma)=\emptyset$. Thus, (BA) holds also in this case.

Suppose now that $\nu^{b / 2} \rho \rtimes \sigma$ reduces for some $b \in \mathbb{N}, b>1$. We shall consider first the case $b \in 2 \mathbb{N}$. Then $\rho \rtimes 1$ or $\nu \rho \rtimes 1$ reduces by (D) and (12-2). Therefore, $L\left(\rho, R_{d_{\rho}}, s\right)$ has no pole at $s=0$. Therefore, $a \in \operatorname{Jord}_{\rho}(\sigma)$ must be odd. Now Theorem 13.2 of [T2] (see also the remark below) implies

$$
\operatorname{Jord}_{\rho}(\sigma)=\{1,3, \ldots, b-1\} .
$$

Therefore, (BA) holds again. Suppose now that $b$ is odd. Then $\nu^{1 / 2} \rho \rtimes 1$ reduces by (D) and (12-2). This implies that $L\left(\rho, R_{d_{\rho}}, s\right)$ has a pole at $s=0$, and further $a \in \operatorname{Jord}_{\rho}(\sigma)$ must be even. Theorem 13.2 of [T2] implies

$$
\operatorname{Jord}_{\rho}(\sigma)=\{2,4, \ldots, b-1\} .
$$

Therefore, (BA) holds.

Assume now that (BA) holds. Let $\nu^{b / 2} \rho \rtimes \sigma$ reduce for some $b \in \mathbb{Z}_{+}$. First consider the case of even $b$. Suppose $\operatorname{Jord}_{\rho}(\sigma)=\emptyset$. Then (BA) implies that $L\left(\rho, R_{d_{\rho}}, s\right)$ has no pole at $s=0$. This implies that $\rho \rtimes 1$ or $\nu \rho \rtimes 1$ reduces. Therefore, (D) holds in this case. Suppose now $\operatorname{Jord}_{\rho}(\sigma) \neq \emptyset$. Then by (BA), from $(b-1+1) / 2=b / 2$ it follows that $b-1 \in \operatorname{Jord}_{\rho}(\sigma)$. Since $b-1$ is odd, $\left(\mathrm{J}-1^{\prime}\right)$ from Remark 2.3 implies that $\rho \rtimes 1$ or $\nu \rho \rtimes 1$ reduces. Again, (D) holds. Now consider the case of odd $b$. Suppose first $\operatorname{Jord}_{\rho}(\sigma)=\emptyset$. Now (BA) implies that $L\left(\rho, R_{d_{\rho}}, s\right)$ has a pole at $s=0$. This implies that $\nu^{1 / 2} \rho \rtimes 1$ reduces. Thus, (D) holds. Suppose now $\operatorname{Jord}_{\rho}(\sigma) \neq \emptyset$. Then again $b-1 \in \operatorname{Jord}_{\rho}(\sigma)$. Since $b-1$ is even, $L\left(\rho, R_{d_{\rho}}, s\right)$ has a pole at $s=0$, and therefore $\nu^{1 / 2} \rho \rtimes 1$ reduces, which implies that (D) holds also in this case. This completes the proof.

We have not proved that (HI) implies (BA). This is certainly not easy (one needs to use the $L$-function defined by Shahidi). If one wants to avoid the use of the $L$ functions (as done in 4.1 of [M5]) in the definition of the Jordan block (this is also done in Remark 14.5 of this paper), (HI) is enough for the definiton of the Jordan block (see 4.2 of [M5]). Therefore, (HI) seems to be the most important property to be proved. This seems to be a difficult problem.

12.2. Remark. Note that in Theorem 13.2 of [T2] we have the assumption char $\left(F^{\prime}\right)$ $=0$. This assumption is used in the proof of that theorem to prove irreducibility. Note that the irreducibility that we needed in the proof of the last lemma is in the unitarizable case. This irreducibility follows in the same way as in the proof of Propositions 4.1 and 4.2. Namely, let $\delta(\Delta)$ be an $F^{\prime} / F$-selfdual irreducible (unitarizable) square integrable representation of a general linear group such that $\rho^{\prime} \rtimes \sigma$ is irreducible for every $\rho^{\prime} \in \Delta$. Then proofs of Propositions 4.1 and 4.2 imply that $\delta(\Delta) \rtimes \sigma$ is irreducible. These proofs do not require $\operatorname{char}\left(F^{\prime}\right)=0$.

We shall now write one direct consequence of the proof of Proposition 12.1. From the (non-negative) reducibility point $\alpha(\rho, \sigma)=b / 2 \in(1 / 2) \mathbb{Z}_{+}$, we can write directly

$$
\operatorname{Jord}_{\rho}(\sigma)=\left\{b-1-2 i ; i \in \mathbb{Z}_{+} \text {and } b-1-2 i \in \mathbb{N}\right\} .
$$


Observe that for the trivial representation we have

$$
\begin{aligned}
& \operatorname{Jord}\left(1_{S O(1)}\right)=\emptyset, \\
& \operatorname{Jord}\left(1_{S p(0)}\right)=\left\{\left(1_{G L(1)}, 1\right)\right\}
\end{aligned}
$$

(depending on the series of the groups with which we are working).

There is one type of $\rho$ for which the problem of computing the reducibility points of induced representations with irreducible cuspidal representations $\sigma$ can be in principle solved using the known facts. It is the case where $\rho$ is a quadratic character. One has to use ideas of Adams, Kudla and Rallis to interpret the reducibility points in terms of the Howe duality ([W2] is an example of this kind). This interpretation is a local analogue of a more difficult global result explained in $\mathrm{KuR}$ (in particular of $6.1 \mathrm{in} \mathrm{KuR}$; see also [M4]). A particular case which is completely written is the case where the cuspidal representation $\sigma$ is quadratic unipotent ([M3]).

\section{TEMPERED REPRESENTATIONS}

We continue to assume (BA) again (until the end of the paper).

Fix an irreducible (unitarizable) square integrable representation

$$
\delta(\rho, a)
$$

of a general linear group (recall that $\delta(\rho, a)$ denotes $\left.\delta\left(\left[\nu^{-(a-1) / 2} \rho, \nu^{(a-1) / 2} \rho\right]\right)\right)$. Let $\pi$ be a similar representation of $S_{q}$.

If $\rho$ is not $F^{\prime} / F$-selfdual, it is well known that

$$
\delta(\rho, a) \rtimes \pi
$$

is irreducible (one can also get this easily from (1-1) and Theorem 4.9 of T1]).

Therefore, to describe the reducibility of $\delta(\rho, a) \rtimes \pi$, it remains to consider the case of $F^{\prime} / F$-selfdual irreducible cuspidal representations $\rho$. We shall now assume that $\rho$ is $F^{\prime} / F$-selfdual.

The composition of the standard intertwining operators

$$
\nu^{s} \delta(\rho, a) \rtimes \pi \rightarrow \nu^{-s} \delta(\rho, a) \rtimes \pi \rightarrow \nu^{s} \delta(\rho, a) \rtimes \pi
$$

can be computed in terms of the $L$-functions using (BA) (such computations are carried out in [M1]). To have simpler notation, we will, here, write $\delta(\rho, a)$ instead of $L\left(\delta\left(\left[\nu^{-(a-1) / 2} \rho, \nu^{(a-1) / 2} \rho\right]\right)\right)$; this is the generalized Steinberg representation of $G L\left(d_{\rho} a\right)$ based on $\rho$. The result is the following products:

$$
\begin{gathered}
\prod_{\left(\rho^{\prime}, a^{\prime}\right) \in \operatorname{Jord}(\pi)} L\left(\delta(\rho, a) \times \delta\left(\rho^{\prime}, a^{\prime}\right), s\right) L\left(\delta(\rho, a) \times \delta\left(\rho^{\prime}, a^{\prime}\right), s+1\right)^{-1,} \\
\prod_{\left(\rho^{\prime}, a^{\prime}\right) \in \operatorname{Jord}(\pi)} L\left(\delta(\rho, a) \times \delta\left(\rho^{\prime}, a^{\prime}\right),-s\right) L\left(\delta(\rho, a) \times \delta\left(\rho^{\prime}, a^{\prime}\right),-s+1\right)^{-1}, \\
L\left(\delta(\rho, a), R_{a d_{\rho}}, 2 s\right) L\left(\delta(\rho, a), R_{a d_{\rho}}, 2 s+1\right)^{-1}, \\
L\left(\delta(\rho, a), R_{a d_{\rho}},-2 s\right) L\left(\delta(\rho, a), R_{a d_{\rho}},-2 s+1\right)^{-1} .
\end{gathered}
$$

Now (J-1) of section 2 is equivalent to the fact that the two last products (13-3) and (13-4) have no pole at $s=0$. The two first products (13-1) and (13-2) are easy 
to analyze using Theorem 8.2 of [JPSS]:

$$
L\left(\delta(\rho, a) \times \delta\left(\rho, a^{\prime}\right), s\right)=\prod_{k=\left|a-a^{\prime}\right| / 2}^{\left(a+a^{\prime}\right) / 2-1} L\left(\rho \times \rho^{\prime}, s+k\right) .
$$

Denominators have no pole and numerators have pole of order one exactly when $(\rho, a) \in \operatorname{Jord}(\pi)$.

This explains the following result: the induced representation

$$
\delta(\rho, a) \rtimes \pi
$$

is irreducible if and only if either $(\rho, a)$ does not satisfy (J-1) of section 2 or $(\rho, a) \in$ $\operatorname{Jord}(\pi)$.

Therefore, $\operatorname{Jord}_{\rho}(\pi)$ determines completely if $\delta(\rho, a) \rtimes \pi$ is reducible or not (for an arbitrary irreducible unitarizable cuspidal representation $\rho$ ).

A more elementary argument can be applied in the following way to obtain the same result. If $a$ satisfies condition (J-1) of section 2, then by definition $\operatorname{Jord}_{\rho}(\pi)$ tells exactly when $\delta(\rho, a) \rtimes \pi$ reduces (this is a part of the definition of $\operatorname{Jord}_{\rho}(\pi)$ ). Suppose that $a$ does not satisfy (J-1). Let $a$ be odd. Then (BA) implies that $\nu^{x} \rho \rtimes \sigma$ reduces for some $x \in(1 / 2)+\mathbb{Z}$. Now Proposition 4.2 of [T2] (together with Remark 12.2 in this paper and (BA)) implies that $\delta(\rho, a) \rtimes \pi$ is irreducible. If we suppose that $a$ is even, we get in a similar way that $\delta(\rho, a) \rtimes \pi$ is irreducible (using Proposition 4.1 of [T2] and Remark 12.2 of this paper).

The computation of the product of standard intertwining operators can be generalized by replacing $\pi$ with a representation induced from an irreducible square integrable representation. This computation can be made since the case of the general linear groups is already known by Shahidi's results. Using the result of HarishChandra, we can compute the intertwining algebra of a representation induced by an irreducible square integrable representation in terms of poles of the standard intertwining operators. From the above description of reducibility of $\delta(\rho, a) \rtimes \pi$, we obtain in that way the following

13.1. Theorem. (i) Let $\rho_{1}, \ldots, \rho_{n}$ be a set of (equivalence classes of) irreducible unitarizable cuspidal representations of general linear groups $G L\left(k_{i}\right), k_{i} \geq 1$, and let $a_{1}, \ldots, a_{n} \in \mathbb{N}$. Suppose that $\pi$ is an irreducible square integrable representation of some $S_{q}$. Then the induced representation

$$
\Pi=\left(\prod_{i=1}^{n} \delta\left(\rho_{i}, a_{i}\right)\right) \rtimes \pi
$$

is a multiplicity one representation of length $2^{m}$, where $m$ is the cardinal number of the following set:

$$
\left\{\left(\rho_{i}, a_{i}\right) ; 1 \leq i \leq n,\left(\rho_{i}, a_{i}\right) \text { satisfies }(J-1) \text { and }\left(\rho_{i}, a_{i}\right) \notin \operatorname{Jord}(\pi)\right\}
$$

(note that we count only different $\left(\rho_{i}, a_{i}\right)$ 's, not the different indexes).

(ii) Suppose that we have another collection $\rho_{1}^{\prime}, \ldots, \rho_{n^{\prime}}^{\prime}, a_{1}^{\prime}, \ldots, a_{n^{\prime}}^{\prime}$ and $\pi^{\prime}$ as above. We define the representation $\Pi^{\prime}$ for this collection in the same way as we have defined $\Pi$ in (13-5) for $\rho_{1}, \ldots, \rho_{n}, a_{1}, \ldots, a_{n}$ and $\pi$. Then the representations $\Pi$ and $\Pi^{\prime}$ have an irreducible subquotient in common, if and only if they are equivalent. This happens if and only if $\pi \cong \pi^{\prime}, n=n^{\prime}$ and $\left(\rho_{1}, a_{1}\right), \ldots,\left(\rho_{n}, a_{n}\right),\left(\rho_{1}^{*}, a_{1}\right)$, $\ldots,\left(\rho_{n}^{*}, a_{n}\right)$ is a permutation of $\left(\rho_{1}^{\prime}, a_{1}\right), \ldots,\left(\rho_{n}^{\prime}, a_{n}\right),\left(\rho_{1}^{\prime *}, a_{1}\right), \ldots,\left(\rho_{n}^{\prime *}, a_{n}\right)$. 
The first part of the theorem can be obtained from the discussion which precedes the theorem, also using Goldberg's result from G (this requires the assumption char $F^{\prime}=0$ ). The second part of the theorem follows from Proposition III.4.1 of W1.

The above theorem gives a reduction of irreducible tempered representations to cuspidal representations and cuspidal reducibilities. Therefore, it implies also the same type of reduction of the non-unitary duals (i.e. of the parameters in the Langlands classification).

\section{EXERCISES OF THE INTRODUCTION AND EXAMPLES OF ADMISSIBLE TRIPLES}

We shall now prove the exercises which we have mentioned in the introduction. Fix Jord, which corresponds to some $\varphi: W_{F} \times S L(2, \mathbb{C}) \rightarrow^{L} G$ (as in the introduction), and fix a function

$$
\epsilon: \text { Jord } \rightarrow\{ \pm 1\}
$$

Using the notation of the introduction, we shall directly construct

$$
\varphi_{\varphi, \epsilon, \text { cusp }}, \epsilon_{\varphi, \epsilon, \text { cusp }}
$$

To do that, we fix $\rho$ such that $\operatorname{Jord}_{\rho} \neq \emptyset$, and we decompose $\operatorname{Jord}_{\rho}$ into a partition

$$
\bigcup_{i=1}^{\ell} S_{i}=\operatorname{Jord}_{\rho}
$$

of non-empty sets $S_{i}$, where $S_{1}, \ldots, S_{\ell}$ are subsets of $\mathbb{N}$, in such a way that for all $1 \leq i<\ell$ and for all $a \in S_{i}, a^{\prime} \in S_{i+1}$ we have $a<a^{\prime}$ and $\epsilon(\rho, a) \neq \epsilon\left(\rho, a^{\prime}\right)$.

Note that the last condition implies that $\epsilon$ is constant on each $S_{i}$ for any $i \in[1, \ell]$. Clearly, one can perform the above decomposition, and there is only one way to perform this.

Define $I \subseteq[1, \ell]$ by: $i \in I$ if and only if card $\left(S_{i}\right)$ is odd and $i \neq 1$ if $\operatorname{Jord}_{\rho} \subseteq 2 \mathbb{N}$ and $\epsilon \mid S_{1}$ is trivial. If $I=\emptyset$, we take $\operatorname{Jord}_{\rho, \text { cusp }}=\emptyset$. If $I \neq \emptyset$, we denote by $\psi$ the unique ordering preserving bijection between $I$ and $[1, \operatorname{card}(I)]$. Define

$$
\operatorname{Jord}_{\rho, \text { cusp }}:=\{(\rho, 2 j-\eta) ; j \in[1, \operatorname{card}(I)]\},
$$

where $\eta=1$ (resp. 0) if $\operatorname{Jord}_{\rho}$ contains odd (resp. even) elements. Define

$$
\epsilon_{\rho, \text { cusp }}: \operatorname{Jord}_{\rho, \text { cusp }} \rightarrow\{ \pm 1\}
$$

with

$$
\epsilon_{\rho, c u s p}(\rho, 2 j-\eta)=\epsilon\left(\rho, a_{j}\right),
$$

where $a_{j}$ is any element in $S_{\psi^{-1}(j)}$.

We define $\varphi_{\varphi, \epsilon, c u s p}$ uniquely by:

$$
\operatorname{Jord}\left(\varphi_{\varphi, \epsilon, c u s p}\right)=\bigcup_{\rho} \operatorname{Jord}_{\rho, c u s p} .
$$

Further, $\epsilon_{\varphi, \epsilon, \text { cusp }}$ comes from all the $\epsilon_{\rho, \text { cusp }}$ in the obvious way. We now need to prove that $\varphi_{\varphi, \epsilon, c u s p}, \epsilon_{\varphi, \epsilon, \text { cusp }}$ is the cuspidal support of $\varphi, \epsilon$. One proves this directly.

The first case is when all $S_{i}$ (as above) have cardinality 1 . This is exactly the case when the first condition of the introduction is satisfied (i.e. we are in the alternated case). In the other case, we argue by induction. The observation here is that the subsets associated to $\varphi_{1}, \epsilon_{1}$ (the notation is the same as in the introduction) are obtained from those associated to $\varphi, \epsilon$ by deleting two elements in the same subset. 
The second exercise of the introduction is also easy to conclude from our construction. Fix $\varphi, \epsilon$ as above, and let $\epsilon^{\prime}$ be such that $\varphi, \epsilon^{\prime}$ is a Langlands parameter. We assume that $\Delta_{\epsilon}=\Delta_{\epsilon^{\prime}}$ and we have to prove that

$$
\varphi_{\varphi, \epsilon, c u s p}=\varphi_{\varphi, \epsilon^{\prime}, \text { cusp }}
$$

The assumption $\Delta_{\epsilon}=\Delta_{\epsilon^{\prime}}$ on the connection between $\epsilon$ and $\epsilon^{\prime}$ implies that, for all $\rho$, the decomposition of $\operatorname{Jord}_{\rho}$ into intersections with segments as above is the same if we use $\epsilon$ instead of $\epsilon^{\prime}$. This and the above construction imply $\varphi_{\varphi, \epsilon, \text { cusp }}=\varphi_{\varphi, \epsilon^{\prime}, \text { cusp }}$.

In the sequel of this section, we shall write a few examples concerning admissible triples. To simplify discussion, we shall assume that our triples in this section satisfy condition (L) of the fifth section.

For a given $J$ ord and $\pi_{\text {cusp }}$ (or $\operatorname{Jord}\left(\pi_{\text {cusp }}\right)$ ), we shall say that a partially defined function $\epsilon$ is admissible if $\epsilon$, together with Jord and $\pi_{c u s p}$, forms an admissible triple.

14.1. We shall first consider the case when $\operatorname{Jord}_{\rho}\left(\pi_{\text {cusp }}\right)=\emptyset$ and $L\left(\rho, R_{d_{\rho}}, s\right)$ has a pole at $s=0$ (the last condition is equivalent to the fact that we are in the even case). Then $\nu^{1 / 2} \rho \rtimes \pi_{\text {cusp }}$ reduces. We shall now discuss some possibilities for $\operatorname{Jord}_{\rho}$.

14.1.0. $\operatorname{Jord}_{\rho}=\emptyset$. Here there is only one $\epsilon$. We are in the alternated case. The attached representation is $\pi_{\text {cusp }}$.

14.1.1. $\operatorname{Jord}_{\rho}=\left\{2 k_{1}\right\}, k_{1} \in \mathbb{N}$. Then there are two possible partial functions $\epsilon$, which we can describe by the following table:

$$
\begin{array}{ccc}
\operatorname{Jord}_{\rho} & \epsilon_{1} & \epsilon_{2} \\
2 k_{1} & 1 & -1 .
\end{array}
$$

First $\epsilon_{2}$ is not admissible $\left(\epsilon_{2}\right.$ cannot be in the mixed case because $\operatorname{card}\left(\operatorname{Jord}_{\rho}\right)=1$, and $\epsilon_{2}$ cannot be alternated since $\operatorname{card}\left(\operatorname{Jord}_{\rho}\right)=1$ and $\left.\operatorname{card}\left(\operatorname{Jord}_{\rho}^{\prime}\left(\pi_{\text {cusp }}\right)\right)=0\right)$. Further,

is admissible and we are in the alternated case.

14.1.2. $\operatorname{Jord}_{\rho}=\left\{2 k_{1}, 2 k_{2}\right\}, k_{1}<k_{2} \in \mathbb{N}$. Then there exist the following functions $\epsilon$ on $\operatorname{Jord}_{\rho}$ :

$$
\begin{array}{lrrrl}
\operatorname{Jord}_{\rho} & \epsilon_{1} & \epsilon_{2} & \epsilon_{3} & \epsilon_{4} \\
2 k_{1} & 1 & 1 & -1 & -1 \\
2 k_{2} & 1 & -1 & 1 & -1 .
\end{array}
$$

We cannot have alternated $\epsilon\left(\operatorname{since} \operatorname{card}\left(\operatorname{Jord}_{\rho}\right)=2\right.$ and $\left.\operatorname{card}\left(\operatorname{Jord}_{\rho}\left(\pi_{\text {cusp }}\right)\right)=0\right)$. Obviously,

$$
\epsilon_{1}, \epsilon_{4}
$$

are admissible (see 14.1.0). 
14.1.3. $\operatorname{Jord}_{\rho}=\left\{2 k_{1}, 2 k_{2}, 2 k_{3}\right\}, k_{1}<k_{2}<k_{3} \in \mathbb{N}$. We have the following possibilities for $\epsilon$ :

$$
\begin{array}{rrrrrrrrl}
\operatorname{Jord}_{\rho} & \epsilon_{1} & \epsilon_{2} & \epsilon_{3} & \epsilon_{4} & \epsilon_{5} & \epsilon_{6} & \epsilon_{7} & \epsilon_{8} \\
2 k_{1} & 1 & 1 & 1 & 1 & -1 & -1 & -1 & -1 \\
2 k_{2} & 1 & 1 & -1 & -1 & 1 & 1 & -1 & -1 \\
2 k_{3} & 1 & -1 & 1 & -1 & 1 & -1 & 1 & -1
\end{array}
$$

We cannot have alternated $\epsilon\left(\right.$ since $\operatorname{card}\left(\operatorname{Jord}_{\rho}\right)=3$ and $\left.\operatorname{card}\left(\operatorname{Jord}_{\rho}\left(\pi_{\text {cusp }}\right)\right)=0\right)$. Thus $\epsilon_{3}$ and $\epsilon_{6}$ cannot be admissible. Further, if we have an odd number of -1 's, then from 14.1.1 we see that $\epsilon$ cannot be admissible. Thus, the following remain:

$$
\epsilon_{1}, \epsilon_{4}, \epsilon_{7} .
$$

From 14.1.1 we see that they are admissible.

14.1.4. $\operatorname{Jord}_{\rho}=\left\{2 k_{1}, 2 k_{2}, 2 k_{3}, 2 k_{4}\right\}, k_{1}<k_{2}<k_{3}<k_{4} \in \mathbb{N}$. We have the following partial functions $\epsilon$ :

$(14-4)$

$$
\begin{aligned}
& \operatorname{Jord}_{\rho} \quad \epsilon_{1} \quad \epsilon_{2} \quad \epsilon_{3} \quad \epsilon_{4} \quad \epsilon_{5} \quad \epsilon_{6} \quad \epsilon_{7} \quad \epsilon_{8} \quad \epsilon_{9} \quad \epsilon_{10} \quad \epsilon_{11} \quad \epsilon_{12} \quad \epsilon_{13} \quad \epsilon_{14} \quad \epsilon_{15} \quad \epsilon_{16} \\
& \begin{array}{llllllllllllllllll}
2 k_{1} & 1 & 1 & 1 & 1 & 1 & 1 & 1 & 1 & -1 & -1 & -1 & -1 & -1 & -1 & -1 & -1
\end{array}
\end{aligned}
$$

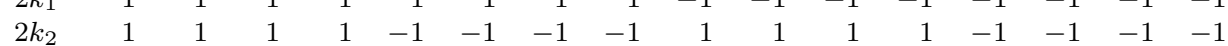

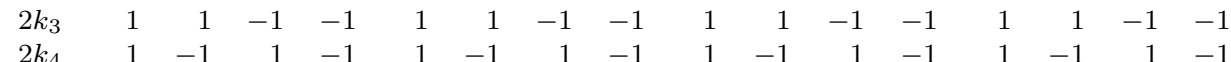

We have not alternated $\epsilon\left(\operatorname{since} \operatorname{card}\left(\operatorname{Jord}_{\rho}\right)=4\right.$ and $\left.\operatorname{card}\left(\operatorname{Jord}_{\rho}\left(\pi_{\text {cusp }}\right)\right)=0\right)$. Thus $\epsilon_{6}$ and $\epsilon_{11}$ cannot be admissible. Again, if we have an odd number of -1 's, then from 14.1.2 we see that $\epsilon$ cannot be admissible. Thus, $\epsilon_{2}, \epsilon_{3}, \epsilon_{5}, \epsilon_{8}, \epsilon_{9}, \epsilon_{12}, \epsilon_{14}$ and $\epsilon_{15}$ are not admissible. The following remain:

$$
\epsilon_{1}, \epsilon_{4}, \epsilon_{7}, \epsilon_{10}, \epsilon_{13}, \epsilon_{16} \text {. }
$$

From 14.1.2 it follows that they are admissible.

14.2. Now we shall consider the case $\operatorname{Jord}_{\rho}\left(\pi_{\text {cusp }}\right)=\{2\}$. Then $\nu^{3 / 2} \rho \rtimes \pi_{\text {cusp }}$ reduces. We shall list some examples for $\operatorname{Jord}_{\rho}$. In paragraphs 14.2.i below, $\epsilon_{j}$ will denote the function $\epsilon_{j}$ from the tables in 14.1.i.

14.2.0. $\operatorname{Jord}_{\rho}=\emptyset$. This case cannot have admissible $\epsilon$ (since there is no bijection between the sets $\emptyset$ and $\left.\operatorname{Jord}_{\rho}\left(\pi_{\text {cusp }}\right)=\{2\}\right)$.

14.2.1. $\operatorname{Jord}_{\rho}=\left\{2 k_{1}\right\}, k_{1} \in \mathbb{N}$. First, $\epsilon_{1}$ is not admissible (since $\epsilon_{1}$ cannot be in the mixed case, and further $\epsilon_{1}$ cannot be alternated since $\operatorname{card}\left(\operatorname{Jord}_{\rho}\right)=1$ and $\left.\operatorname{card}\left(\operatorname{Jord}_{\rho}^{\prime}\left(\pi_{\text {cusp }}\right)\right)=2\right)$. For

$$
\epsilon_{2}
$$

we are in the alternated case. If $k_{1}=1$, the attached representation is $\pi_{\text {cusp }}$.

14.2.2. $\operatorname{Jord}_{\rho}=\left\{2 k_{1}, 2 k_{2}\right\}, k_{1}<k_{2} \in \mathbb{N}$. Here we cannot have $\epsilon$ of mixed type, because of 14.2.0. Now consider alternated $\epsilon$. Suppose that $\epsilon_{3}$ is admissible. Then we would have a bijection of $\operatorname{Jord}_{\rho}$ onto $\{2\}$, which is impossible. For $\epsilon_{2}$, we have a bijection of $\operatorname{Jord}_{\rho}$ onto $\{0,2\}$. Thus

is admissible and we are in the alternated case. 
14.2.3. $\operatorname{Jord}_{\rho}=\left\{2 k_{1}, 2 k_{2}, 2 k_{3}\right\}, k_{1}<k_{2}<k_{3} \in \mathbb{N}$. We cannot have alternated $\epsilon$. Therefore, $\epsilon_{3}$ and $\epsilon_{6}$ cannot be admissible. Further, if we have an odd number of 1 's, then from 14.2.1 we see that $\epsilon$ cannot be admissible. Thus, the following remain:

$$
\epsilon_{2}, \epsilon_{5}, \epsilon_{8}
$$

Now 14.2.1 implies that they are admissible.

14.2.4. $\operatorname{Jord}_{\rho}=\left\{2 k_{1}, 2 k_{2}, 2 k_{3}, 2 k_{4}\right\}, k_{1}<k_{2}<k_{3}<k_{4} \in \mathbb{N}$. We do not have alternated $\epsilon$ here. Thus $\epsilon_{6}$ and $\epsilon_{11}$ cannot be admissible. Again, if we have an even number of 1's, then from 14.2.2 we see that $\epsilon$ cannot be admissible. Thus, $\epsilon_{1}, \epsilon_{4}, \epsilon_{6}, \epsilon_{7}, \epsilon_{10}, \epsilon_{11}, \epsilon_{13}$ and $\epsilon_{16}$ are not admissible. Among the $\epsilon$ 's which remain, 14.2.2 implies that after deleting 1,1 , or $-1,-1$, we need to have $1,-1$ left (in this order). Therefore, all the candidates for admissible $\epsilon$ 's are among

$$
\epsilon_{2}, \epsilon_{5}, \epsilon_{8}, \epsilon_{14}
$$

Their admissibility follows from 14.2.2.

14.3. Now suppose that $L\left(\rho, R_{d_{\rho}}, s\right)$ has no pole at $s=0$ (i.e. we are in the odd case). Let $\operatorname{Jord}_{\rho}\left(\pi_{\text {cusp }}\right)=\emptyset$ (then $\rho \rtimes \pi$ reduces). Now if one changes $2 k_{i}$ into $2 k_{i}-1$ in examples 14.1.i, one gets admissible $\epsilon$ 's in this case, assuming that i is even (i.e. $\operatorname{Jord}_{\rho}$ has an even number of elements). If $\operatorname{Jord}_{\rho}$ has an odd number of elements, then there are no admissible partial functions in this case.

14.4. If $\operatorname{Jord}_{\rho}\left(\pi_{\text {cusp }}\right)=\{1,3, \ldots, 2 l-1\}$ for some $l \in \mathbb{N}$, then $\nu^{l} \rho \rtimes \sigma$ reduces. We shall now consider the case $l=1$, i.e. $\operatorname{Jord}_{\rho}\left(\pi_{\text {cusp }}\right)=\{1\}$.

14.4.0 $\operatorname{Jord}_{\rho}=\emptyset$. Here we cannot have admissible $\epsilon$ (otherwise we would have a bijection between the sets $\emptyset$ and $\left.\operatorname{Jord}_{\rho}\left(\pi_{\text {cusp }}\right)=\{2\}\right)$.

14.4.1. $\operatorname{Jord}_{\rho}=\left\{2 k_{1}-1\right\}, k_{1} \in \mathbb{N}$. There is only one

$$
\epsilon=\emptyset
$$

and it is admissible of alternated type.

14.4.2. $\operatorname{Jord}_{\rho}=\left\{2 k_{1}-1,2 k_{2}-1\right\}, k_{1}<k_{2} \in \mathbb{N}$. We have the following partial functions $\epsilon$ which we shall describe in the following way:

$$
\begin{array}{cccc}
\operatorname{Jord}_{\rho} & \epsilon_{1} & \epsilon_{2} \\
2 k_{1}-1 & & \\
& 1 & -1 \\
2 k_{2}-1 & &
\end{array} .
$$

Here $\epsilon_{1}$ and $\epsilon_{2}$ denote the following partial functions:

$$
\begin{gathered}
\epsilon_{1}\left(2 k_{1}-1\right) \epsilon_{1}\left(2 k_{2}-1\right)^{-1}=1, \\
\epsilon_{2}\left(2 k_{1}-1\right) \epsilon_{2}\left(2 k_{2}-1\right)^{-1}=-1 .
\end{gathered}
$$

Note that we cannot have $\epsilon$ of mixed type (because of 14.4.0). Further, $\epsilon_{2}$ is not alternated $\left(\operatorname{since} \operatorname{card}\left(\operatorname{Jord}_{\rho}\right)=2\right.$ and $\left.\operatorname{card}\left(\operatorname{Jord}_{\rho}\left(\pi_{\text {cusp }}\right)\right)=1\right)$. Therefore, in this case we do not have admissible $\epsilon$ 's. 
14.4.3. $\operatorname{Jord}_{\rho}=\left\{2 k_{1}-1,2 k_{2}-1,2 k_{3}-1\right\}, k_{1}<k_{2}<k_{3} \in \mathbb{N}$. We have the following partial functions $\epsilon$ :

$$
\begin{array}{ccccc}
\operatorname{Jord}_{\rho} & \epsilon_{1} & \epsilon_{2} & \epsilon_{3} & \epsilon_{4} \\
2 k_{1}-1 & & & & \\
2 k_{2}-1 & 1 & 1 & -1 & -1 \\
2 k_{3}-1 & 1 & -1 & 1 & -1
\end{array}
$$

The interpretation of the table is analogous to the interpretation of the table in 14.4.2. First, we cannot have $\epsilon$ of alternated type by the usual argument. Thus, the following remain:

$$
\epsilon_{1}, \epsilon_{2}, \epsilon_{3} .
$$

As before, 14.4.1 implies that the above $\epsilon$ 's are admissible.

14.4.4. $\operatorname{Jord}_{\rho}=\left\{2 k_{1}-1,2 k_{2}-1,2 k_{3}-1,2 k_{4}-1\right\}, k_{1}<k_{2}<k_{3}<k_{4} \in \mathbb{N}$. Obviously, we cannot have alternated $\epsilon$ here. Now 14.4.2 (or 14.4.0) implies that we also do not have $\epsilon$ of mixed type here.

14.4.5. $\operatorname{Jord}_{\rho}=\left\{2 k_{1}-1,2 k_{2}-1,2 k_{3}-1,2 k_{4}-1,2 k_{5}-1\right\}, k_{1}<k_{2}<k_{3}<k_{4}<$ $k_{5} \in \mathbb{N}$. We have the following partial functions $\epsilon$ :

$$
\begin{array}{ccccccccccccccccc}
\operatorname{Jord}_{\rho} & \epsilon_{1} & \epsilon_{2} & \epsilon_{3} & \epsilon_{4} & \epsilon_{5} & \epsilon_{6} & \epsilon_{7} & \epsilon_{8} & \epsilon_{9} & \epsilon_{10} & \epsilon_{11} & \epsilon_{12} & \epsilon_{13} & \epsilon_{14} & \epsilon_{15} & \epsilon_{16} \\
2 k_{1}-1 & & & & & & & & & & & & & & & & \\
2 k_{2}-1 & 1 & 1 & 1 & 1 & 1 & 1 & 1 & 1 & -1 & -1 & -1 & -1 & -1 & -1 & -1 & -1 \\
2 k_{3}-1 & 1 & 1 & 1 & 1 & -1 & -1 & -1 & -1 & 1 & 1 & 1 & 1 & -1 & -1 & -1 & -1 \\
2 k_{4}-1 & 1 & 1 & -1 & -1 & 1 & 1 & -1 & -1 & 1 & 1 & -1 & -1 & 1 & 1 & -1 & -1 \\
2 k_{5}-1 & 1 & -1 & 1 & -1 & 1 & -1 & 1 & -1 & 1 & -1 & 1 & -1 & 1 & -1 & 1 & -1 \\
\end{array}
$$

First, we do have not alternated $\epsilon$. Thus, $\epsilon_{16}$ is not admissible. Further, if we remove 1 , the resulting restricted partial function that we then obtain cannot be $-1,-1$ by 14.4.2. Therefore, the candidates for admissible $\epsilon$ 's reduce to

$$
\epsilon_{1}, \epsilon_{2}, \epsilon_{3}, \epsilon_{5}, \epsilon_{6}, \epsilon_{7}, \epsilon_{9}, \epsilon_{11}, \epsilon_{12}, \epsilon_{14} .
$$

Their admissibility follows from 14.4.2.

14.4.6. $\operatorname{Jord}_{\rho}=\left\{2 k_{1}-1,2 k_{2}-1, \ldots, 2 k_{2 n}-1\right\}, k_{1}<k_{2}<\cdots<k_{2 n} \in \mathbb{N}$. Again, we do not have $\epsilon$ of alternated type here. As before, we see that we do not have $\epsilon$ of mixed type here.

14.5. Remark. Here we shall give a slightly modified interpretation of the classification of irreducible square integrable representations.

(i) Fix an irreducible cuspidal representation $\pi_{\text {cusp }}$ of $S_{n^{\prime}}$. Let $\rho_{1}, \ldots, \rho_{k}$ be inequivalent $F^{\prime} / F$-selfdual irreducible cuspidal representations of general linear groups. Let $\nu^{\mathbb{R}} \rho_{i}=\left\{\nu^{x} \rho_{i} ; x \in \mathbb{R}\right\}$. Denote by

$$
\mathcal{D}\left(\rho_{1}, \ldots, \rho_{k} ; \pi_{\text {cusp }}\right)
$$


the set of all equivalence classes of irreducible square integrable representations of groups $S_{n}$, whose factors are all contained in $\bigcup_{i=1}^{k} \nu^{\mathbb{R}} \rho_{i}$ and whose partial cuspidal support is $\pi_{\text {cusp }}$. These classes are precisely all the (classes of) irreducible square integrable subquotients of all possible $\tau_{1} \times \tau_{2} \times \cdots \times \tau_{\ell} \rtimes \pi_{\text {cusp }}$ when $\ell$ runs over the non-negative integers and $\tau_{1}, \tau_{2}, \ldots, \tau_{l}$ run over $\bigcup_{i=1}^{k} \nu^{\mathbb{R}} \rho_{i}$.

Each irreducible square integrable representation of a group $S_{n}$ is contained in some $\mathcal{D}\left(\rho_{1}, \rho_{2}, \ldots, \rho_{k} ; \pi_{\text {cusp }}\right)$ as above (for some $\left.\pi_{\text {cusp }}\right)$. Therefore, for the classification of irreducible square integrable representations (of the series of the groups that we are considering), it is enough to classify sets $\mathcal{D}\left(\rho_{1}, \rho_{2}, \ldots, \rho_{k} ; \pi_{\text {cusp }}\right)$, and know what the intersection is of such different sets. The problem of describing intersections is very easy. Let

$$
\left\{\rho_{1}, \rho_{2}, \ldots, \rho_{\ell}\right\} \cap\left\{\rho_{1}^{\prime}, \rho_{2}^{\prime}, \ldots, \rho_{\ell^{\prime}}^{\prime}\right\}=\left\{\rho_{1}^{\prime \prime}, \rho_{2}^{\prime \prime}, \ldots, \rho_{\ell^{\prime \prime}}^{\prime \prime}\right\} .
$$

Then

$$
\mathcal{D}\left(\rho_{1}, \rho_{2}, \ldots, \rho_{\ell} ; \pi_{\text {cusp }}\right) \cap \mathcal{D}\left(\rho_{1}^{\prime}, \rho_{2}^{\prime}, \ldots, \rho_{\ell^{\prime}}^{\prime} ; \pi_{\text {cusp }}\right)=\mathcal{D}\left(\rho_{1}^{\prime \prime}, \rho_{2}^{\prime \prime}, \ldots, \rho_{\ell^{\prime \prime}}^{\prime \prime} ; \pi_{\text {cusp }}\right) .
$$

If $\pi_{\text {cusp }}$ is not isomorphic to $\pi_{\text {cusp }}^{\prime}$, then

$$
\mathcal{D}\left(\rho_{1}, \rho_{2}, \ldots, \rho_{\ell} ; \pi_{\text {cusp }}\right) \cap \mathcal{D}\left(\rho_{1}^{\prime}, \rho_{2}^{\prime}, \ldots, \rho_{\ell^{\prime}}^{\prime} ; \pi_{\text {cusp }}^{\prime}\right)=\emptyset .
$$

(ii) We shall give now a useful reduction of a problem of classifying sets $\mathcal{D}\left(\rho_{1}, \ldots, \rho_{k} ; \pi_{\text {cusp }}\right)$.

Let $\pi \in \mathcal{D}\left(\rho_{1}, \ldots, \rho_{k} ; \pi_{\text {cusp }}\right)$. For each $j$, there exists an irreducible representation $\pi_{j}$ of some $S_{n_{j}}$ whose factors are all contained in $\nu^{\mathbb{R}} \rho_{j}$, and there exists an irreducible representation $\tau_{j}$ of a general linear group whose cuspidal support consists of representations from $\left(\bigcup_{i=1}^{k} \nu^{\mathbb{R}} \rho_{i}\right) \backslash \nu^{\mathbb{R}} \rho_{j}$, such that we have an embedding

$$
\pi \hookrightarrow \tau_{j} \rtimes \pi_{j} .
$$

By [Jn1, representations $\pi_{1}, \ldots, \pi_{k}$ are uniquely determined by $\pi$, they are all square integrable and $\pi \mapsto\left(\pi_{1}, \ldots, \pi_{k}\right)$ is a bijection from $\mathcal{D}\left(\rho_{1}, \ldots, \rho_{k} ; \pi_{\text {cusp }}\right)$ onto the Cartesian product $\prod_{i=1}^{k} \mathcal{D}\left(\rho_{i} ; \pi_{\text {cusp }}\right)$. In this way one gets a reduction of the problem of the classification of irreducible square integrable representations to the problem of the classification of sets

$$
\mathcal{D}\left(\rho ; \pi_{\text {cusp }}\right)
$$

(of irreducible square integrable representations; this reduction is implicit in our construction).

(iii) We shall now analyze parameters of representations in $\mathcal{D}\left(\rho ; \pi_{\text {cusp }}\right)$. For $\pi \in \mathcal{D}\left(\rho ; \pi_{\text {cusp }}\right)$ we have

$$
\operatorname{Jord}_{\rho^{\prime}}(\pi)=\operatorname{Jord}_{\rho^{\prime}}\left(\pi_{\text {cusp }}\right)
$$

for any $F / F^{\prime}$-selfdual irreducible cuspidal representation $\rho^{\prime}$ of a general linear group, which is not equivalent to $\rho$. Recall that $\pi_{\text {cusp }}$ is fixed. From the above observations it follows that the parameter $\left(\operatorname{Jor} d(\pi), \epsilon_{\pi}, \pi_{\text {cusp }}\right)$ of $\pi$ is completely determined by the pair $\left(\operatorname{Jord}_{\rho}(\pi), \epsilon_{\pi}\right)$. Further, from the construction of irreducible square integrable representations we see that $\pi$ is determined by $\operatorname{Jord}_{\rho}(\pi)$ and the "restriction" of $\epsilon_{\pi}$ to $\left\{(\rho, a) ; a \in \operatorname{Jord}_{\rho}(\pi)\right\}$. We shall identify this "restriction" of $\epsilon_{\pi}$ with a partially defined function on $\operatorname{Jord}_{\rho}(\pi)$ (since $\left\{(\rho, a) ; a \in \operatorname{Jord}_{\rho}(\pi)\right\}$ and $\operatorname{Jord}_{\rho}(\pi)$ are in a natural bijection), which we shall denote by $\epsilon_{\pi}^{(\rho)}$. 
Therefore the pairs $\operatorname{Jord}_{\rho}$ and $\epsilon^{(\rho)}$, where $\epsilon^{(\rho)}$ is a partially defined function on $\operatorname{Jord}_{\rho}$ which, with $\operatorname{Jord}_{\rho}(\pi)$ and $\pi_{\text {cusp }}$ (in fact with $\operatorname{Jord}_{\rho}\left(\pi_{\text {cusp }}\right)$ ), makes an admissible triple, parameterize $\mathcal{D}\left(\rho ; \pi_{\text {cusp }}\right)$. Note that $\operatorname{Jord}_{\rho}(\pi)$ and $\operatorname{Jord}_{\rho}\left(\pi_{\text {cusp }}\right)$ are finite subsets of $\mathbb{N}$.

(iv) We shall now explicitly describe the parameters $\left(\operatorname{Jord}_{\rho}(\pi), \epsilon^{(\rho)}\right)$ of $\mathcal{D}\left(\rho ; \pi_{\text {cusp }}\right)$ from (iii) (note that this description will not involve $L$-functions). Suppose that $\nu^{ \pm \alpha} \rho \rtimes \pi_{\text {cusp }}$ reduces $(\alpha \geq 0)$. Recall that (BA) implies $\alpha \in(1 / 2) \mathbb{Z}$. Now $\operatorname{Jord}_{\rho}\left(\pi_{\text {cusp }}\right)=\left\{2 \alpha-1-2 i ; i \in \mathbb{Z}_{+}\right.$and $\left.2 \alpha-1-2 i \in \mathbb{N}\right\}$ (see section 12).

Now $\operatorname{Jord}_{\rho}$ of alternated type is a subset of $\mathbb{N}$ consisting of the element of the same parity as $2 \alpha-1$, of cardinality $\alpha$ if $\alpha \in \mathbb{Z}_{+}$and $\alpha \pm 1 / 2$ if $\alpha \notin \mathbb{Z}_{+}$. In both cases, $\epsilon^{(\rho)}$ is uniquely determined with $\operatorname{Jord}_{\rho}$ (and the fact that we are in the alternated case; it is easy to write down this function).

Fix alternated $\operatorname{Jord}_{\rho}$ (and $\epsilon^{(\rho)}$ ). Take any two numbers $a_{-}<a \in \mathbb{N}$ of the same parity as $2 \alpha-1$, such that $\left[a_{-}, a\right] \cap \operatorname{Jord}_{\rho}=\emptyset$. Set $\operatorname{Jord}_{\rho}^{(1)}=\operatorname{Jord}_{\rho} \cup$ $\left\{a_{-}, a\right\}$. Denote by $\epsilon_{1}^{(\rho)}$ any extension (as a partially defined function) of $\epsilon^{(\rho)}$ to $\operatorname{Jord}_{\rho}^{(1)}$ such that $\epsilon^{(\rho)}\left(a_{-}\right)=e^{(\rho)}(a)$ (there are precisely two such extensions; it is easy to write them down). Repeating the above construction again, we will get some $\operatorname{Jord}_{\rho}^{(2)}, \epsilon_{2}^{(\rho)}$. We can continue this construction further. By this simple construction, each admissible $\operatorname{Jord}_{\rho}, \epsilon^{(\rho)}$ can be obtained in finitely many steps (starting from appropriate alternated $J_{o r d}^{+}$).

From the above description, one can see that it is very easy to describe the parameters of $\mathcal{D}\left(\rho ; \pi_{\text {cusp }}\right)$ from the reducibility point $\alpha$ (they are expressed in terms of finite subsets of $\mathbb{N}$ ). The corresponding irreducible square integrable representations are also described with a parallel inductive construction (corresponding to the construction of $\left(\operatorname{Jord}_{\rho}^{(1)}, \epsilon_{1}^{(\rho)}\right),\left(\operatorname{Jord}_{\rho}^{(2)}, \epsilon_{2}^{(\rho)}\right), \ldots$, which started from some alternated $\left.\left(\operatorname{Jord}_{\rho}, \epsilon^{(\rho)}\right)\right)$.

\section{UNITARY GROUPS}

In this section we shall explain necessary modifications which one needs for the classifications obtained for symplectic and odd-orthogonal groups in former sections to also hold for unitary groups.

Fix a series $S_{n}$ of unitary groups (see the first section). Note that $S_{n}$ are connected reductive groups over $F$. Further, formula (1-1) holds (and therefore (1-2) also holds). This follows in a similar way as in the case of non-split odd-orthogonal groups (the reduced root system is either of type $B$ or $C$; in both cases the Weyl group is the same as in the symplectic and odd-orthogonal cases).

Casselman's square integrability criterion has the same form as in the case of symplectic and odd-orthogonal groups. This follows from the description of the positive simple roots (analogously as in section 6 of [T6] for symplectic groups).

In the definition of an admissible triple, we need only specify which $L$-function we need to take in the definition of the parity of $a$ for $(\rho, a) \in J$ ord . The $L$-function is determined by the representation. We shall recall the representation introduced in M2 which enters the definition of the parity.

Let $S_{n}$ be the unitary group of the unitary space $V_{n}$ from the Witt tower and denote $n^{*}=\operatorname{dim}_{F^{\prime}}\left(V_{n}\right)$ (note that $n^{*}=2 n$ if $\operatorname{dim}_{F^{\prime}}\left(V_{n}\right)$ is even, and $n^{*}=2 n+1$ otherwise; recall that $S_{n}$ is the unitary group $U\left(n^{*}, F^{\prime} / F\right)$ ). 
Take an irreducible $F^{\prime} / F$-selfdual cuspidal representation $\rho$ of a general linear group $G L\left(d_{\rho}, F^{\prime}\right)$. The $L$-group of the $F$-group $G L\left(d_{\rho}, F^{\prime}\right)$ is isomorphic to a semidirect product

$$
\left(G L\left(d_{\rho}, \mathbb{C}\right) \times G L\left(d_{\rho}, \mathbb{C}\right)\right)<\operatorname{Gal}\left(F^{\prime} / F\right),
$$

where (the non-trivial element of) $\operatorname{Gal}\left(F^{\prime} / F\right)$ acts on the normal subgroup $G L\left(d_{\rho}, \mathbb{C}\right)$ $\times G L\left(d_{\rho}, \mathbb{C}\right)$ by

$$
\theta\left(g_{1}, g_{2}, 1\right) \theta^{-1}=\left({ }^{t} g_{2}^{-1},{ }^{t} g_{1}^{-1}, 1\right)
$$

(here ${ }^{t} g$ denotes the transposed matrix of $g$ ).

For $\eta \in\{ \pm 1\}$, denote by $R_{d_{\rho}}^{(\eta)}$ the representation of the above $L$-group of $G L\left(d_{\rho}, F^{\prime}\right)$ on $\operatorname{End}_{\mathbb{C}}\left(\mathbb{C}^{d_{\rho}}\right)$ given by:

$$
\left(g_{1}, g_{2}, 1\right) u=g_{1} u^{t} g_{2} \quad \text { and } \quad(1,1, \theta) u=\eta^{t} u .
$$

Suppose now that $S_{n}$ is a series of groups such that the $\operatorname{dimensions} \operatorname{dim}_{F^{\prime}}\left(V_{n}\right)$ are even. Then we shall denote by

$$
R_{d_{\rho}}
$$

the representation $R_{d_{\rho}}^{(1)}$. Otherwise, in the odd case, $R_{d_{\rho}}$ will denote $R_{d_{\rho}}^{(-1)}$.

With $R_{d_{\rho}}$ defined in this way for unitary groups, we have the same definition of the parity as in the second section.

The degree

$$
\sum_{(\rho, a) \in \operatorname{Jord}(\pi)} a d_{\rho}
$$

needs to be $n^{*}$, for an irreducible square integrable representation $\pi$ of $S_{n}$.

These are required modifications in the unitary case.

\section{EvEn-ORTHOGONAL GROUPS}

Fix a series $S_{n}$ of even-orthogonal groups (see the first section). First we need to describe $L$-functions which enter the definition of Jordan blocks. For an irreducible cuspidal selfdual representation $\rho$ of $G L\left(d_{\rho}, F\right)$, we denote by $R_{d_{\rho}}$ the representation of $G L\left(d_{\rho}, \mathbb{C}\right)$ on $\wedge^{2} \mathbb{C}^{d_{\rho}}$.

The degree $\sum_{(\rho, a) \in \operatorname{Jord}(\pi)} a d_{\rho}$ needs to be $2 n$ here, for an irreducible square integrable representation $\pi$ of $S_{n}$.

Denote the subgroup of elements in $S_{n}$ of determinant one by $S_{n}^{\prime}\left(S_{n}^{\prime}\right.$ has index two in $S_{n}$ ).

Now we shall comment on the case of non-split even-orthogonal groups $S_{n}$. Then the Weyl group of $S_{n}^{\prime}$ is the same as in the case of symplectic and odd-orthogonal groups (the root system is of type $B$ ). Therefore, we can apply the calculations done in section 4 of [T5] to the groups $S_{n}^{\prime}$. Further, one can easily see that the analogue of Lemma 5.1 holds here (recall that the unipotent radicals in $S_{n}$ are already contained in $S_{n}^{\prime}$ ). From this one gets that formula (1-1) holds also for groups $S_{n}$.

Further, since we have the same root system as in the case of odd-orthogonal groups, Casselman's square integrability criterion holds for groups $S_{n}^{\prime}$ (and $S_{n}$ ) in the same form as in the case of symplectic and odd-orthogonal groups. 
These are the only comments that we need in the case of non-split even-orthogonal groups.

Suppose now that $S_{n}$ consists of split even-orthogonal groups. Now formula (1-1) holds by $\mathrm{B}$ (the strategy of proving (1-1) requires modification in this case, since the root systems of the groups $S_{n}^{\prime}$ are of type $D$, and the Weyl group is slightly different from the previous cases; there are also other ways to prove (1-1), different from the proof in $[\mathrm{B}]$ ).

A possible difference with the case of the groups that we have studied before appear if $\pi_{\text {cusp }}$ is a representation of $S_{0}=\{1\}$. In this case we have two standard "Siegel parabolic subgroups" in $S_{n}$. They are also parabolic subgroups in $S_{n}$, but they are conjugated in $S_{n}$. Therefore, we can proceed in this situation in the same way as in the cases of groups that we have considered before.

For $n \neq 1$, a (finite length) representation $\pi_{\text {cusp }}$ of $S_{n}$ is cuspidal if $\pi_{\text {cusp }} \mid S_{n}^{\prime}$ is a cuspidal representation of $S_{n}^{\prime}$. The group $S_{1}$ does not have cuspidal representations.

Further, if $n \neq 1$, then a representation $\pi$ of $S_{n}$ is square integrable if and only if $\pi \mid S_{n}^{\prime}$ is a square integrable representation of $S_{n}^{\prime}$. One directly sees that $S_{1}$ does not have square integrable representations (nor has essentially square integrable representations).

A comment regarding Casselman's square integrability criterion is necessary, since the root systems of $S_{n}^{\prime}$ are different from the previous ones. First we shall say a few words about parabolic subgroups.

Denote by $s \in S_{n}$ a quasi-diagonal matrix

$$
\mathrm{q}-\operatorname{diag}(\underbrace{1, \ldots, 1}_{n-1 \text { times }},\left[\begin{array}{ll}
0 & 1 \\
1 & 0
\end{array}\right], \underbrace{1, \ldots, 1}_{n-1 \text { times }}) \text {. }
$$

Recall that standard parabolic subgroups in $S O(2 n, F)$ are parameterized by partitions $\beta=n_{1}+\cdots+n_{k}$ of $0 \leq m \leq n, m \neq n-1$, into a sum of positive integers. Besides these standard parabolic subgroups, the remaining ones are parabolic subgroups $s P_{\beta} s$, when $n=m$ and $n_{k} \geq 2$ (see [B] ).

Let $(\pi, V)$ be an irreducible representation of $S_{n}$, whose partial support is $\pi_{\text {cusp }}$. Denote $V(N)=\operatorname{span}_{\mathbb{C}}\{\pi(n) v-v ; n \in N, v \in V\}$ and let $V_{N}=V / V(N)$ be the normalized Jacquet module.

To check square integrability of $\pi$, we need to check Casselman's square integrability criterion for parabolic subgroups of type $P_{\gamma}$ or $s P_{\gamma} s$, for which Jacquet modules are cuspidal.

Now the Levi factor of a parabolic subgroup $M_{\gamma}$ of $P_{\gamma}$ is naturally isomorphic to $G L\left(n_{1}, F\right) \times \cdots \times G L\left(n_{k}, F\right) \times S O(n-m, F)$. In the case of $s P_{\gamma} s$, it is also naturally isomorphic to $G L\left(n_{1}, F\right) \times \cdots \times G L\left(n_{k}, F\right) \times S O(n-m, F)$, by the conjugation of this subgroup with $s$ (in the first case, we shall say that we are in the non-conjugate situation, while in the other case we shall say that we are in the conjugate situation). Let $\rho=\rho_{1} \otimes \cdots \otimes \rho_{k} \otimes \sigma$ be an irreducible cuspidal subquotient of the Jacquet module, or its conjugate, if we are in the conjugated situation. Define

$$
e_{*}(\rho)=(\underbrace{e\left(\rho_{1}\right), \ldots, e\left(\rho_{1}\right)}_{n_{1} \text { times }}, \ldots, \underbrace{e\left(\rho_{k}\right), \ldots, e\left(\rho_{k}\right)}_{n_{k} \text { times }}, \underbrace{e(\sigma), \ldots, e(\sigma)}_{n-m \text { times }}) \text {, }
$$

where we take $e(\sigma)=0$ if $n-m \geq 2$. 
First consider the case $n-m \geq 2$. Casselman's criterion for square integrability tells us in this case that the square integrability is equivalent to

$$
\sum_{i=1}^{j} e\left(\rho_{i}\right) n_{i}>0, \quad j=1, \ldots, k .
$$

These are our usual relations for square integrability.

Let $n=m$. Suppose $n_{k}=1$. Then the last two simple roots of the root system $D_{n}$ are not in the roots that define the parabolic subgroup. The square integrability criterion now is equivalent to the following relations:

$$
\begin{gathered}
\sum_{i=1}^{j} e\left(\rho_{i}\right) n_{i}>0, \quad j=1, \ldots, k-2, \\
\left(\sum_{i=1}^{k-1} e\left(\rho_{i}\right) n_{i}\right)-e\left(\rho_{k}\right) n_{k}>0 \\
\sum_{i=1}^{k} e\left(\rho_{i}\right) n_{i}>0 .
\end{gathered}
$$

Summing (16-3) and (16-4) we get

$$
\sum_{i=1}^{k-1} e\left(\rho_{i}\right) n_{i}>0 .
$$

Thus

$$
\sum_{i=1}^{j} e\left(\rho_{i}\right) n_{i}>0, \quad j=1, \ldots, k .
$$

Note that (16-6) represents our usual relations for square integrability. We need to see that they are enough for square integrability, i.e. that they also imply (16-3) (recall that $\pi$ is a representation of $O(2 n, F)$ ).

Suppose that (16-6) holds for each irreducible cuspidal subquotient $\rho$ of the Jacquet module. We shall now see that (16-3) holds. We know that there is an epimorphism $V \rightarrow V_{N_{\gamma}} \rightarrow \rho=\rho_{1} \otimes \cdots \otimes \rho_{k} \otimes 1$ of $P_{\gamma}$-representations (the unipotent radical is assumed to act trivially in the last representation). Now conjugating this epimorphism by $s$, we get that there is a $P_{\gamma}$ epimorphism onto $\rho_{1} \otimes \cdots \otimes \rho_{k-1} \otimes \tilde{\rho}_{k} \otimes 1$. Now (16-4) applied to the last subquotient of the Jacquet module implies that (16-3) holds.

It remains to consider the case $n=m$ and $n_{k} \geq 2$. Then $\sigma=\pi_{c u s p}$ is the trivial representation (of $O(0, F)$ ). Now the square integrability criterion gives relations (16-6). Note that these relations need to hold in the non-conjugate situations, as well as in conjugate ones.

Suppose that relations (16-6) hold for non-conjugate situations only. Let $\rho$ be a subquotient of the Jacquet module for $s P_{\gamma} s$ (note $N_{\gamma}=s N_{\gamma} s$ ). Then we have an epimorphism $V \rightarrow V_{N_{\gamma}} \rightarrow s \rho s=s\left(\rho_{1} \otimes \cdots \otimes \rho_{k} \otimes 1\right) s \cong \rho=\rho_{1} \otimes \cdots \otimes s\left(\rho_{k} \otimes 1\right) s$ of $s P_{\gamma} s$-representations (the unipotent radical is assumed to act trivially in the last representation, as in the previous case). Conjugating this epimorphism by $s$, we obtain an epimorphism from $V$ onto $\rho_{1} \otimes \cdots \otimes \rho_{k} \otimes 1$ of $P_{\gamma}$-representations. 
Now relations (16-6) applied to the last subquotient of the Jacquet module (in the non-conjugate situation) imply that (16-6) also hold in the conjugated situation.

At several places, we have used Harish-Chandra's results on the Plancherel measure, especially in $\mathbf{2 . 2}$; this was written in W1. only for connected groups, but we have extended what we need for even-orthogonal groups in the appendix of [M2]. The proof of $\mathbf{2 . 2}$ is already in $\underline{\mathrm{M} 2}$.

\section{REFERENCES}

[Ad] Adams, J., L-functoriality for dual pairs, Astérisque 171-172 (1989), 85-129. MR 91e:22020

[Ar] Arthur, J., Unipotent automorphic representations: global motivations, Automorphic forms, Shimura varieties and L-functions, Progress in Mathematics 10, Birkhäuser, 1990, pp. 1-75. MR 92a:11059

[B] Ban, D., Parabolic induction and Jacquet modules of representations of $O(2 n, F)$, Glasnik Mat. 34(54) (3) (1999), 147-185. MR 2001m:22033

[B-Z] Bernstein I.N., Zelevinsky A.V., Induced Representations of Reductive p-adic groups I, Ann. Sci. École Norm. Sup 10 (1977), 441-472. MR 58:28310

[G] Goldberg, D., Reducibility of induced representations for $S p(2 n)$ and $S O(n)$, Amer. J. Math. 116 (5) (1994), 1101-1151. MR 95g:22016

[JPSS] Jacquet, H.; Piateteski-Shapiro I. and Shalika J., Rankin-Selberg convolutions, Amer. J. Math. 105 (1983), 367-463. MR 85g:11044

[Jn1] Jantzen, C., On supports of induced representations for symplectic and odd-orthogonal groups, Amer. J. Math. 119 (1997), 1213-1262. MR 99b:22028

[Jn2] - On square integrable representations of classical p-adic groups II, Representation Theory 4 (2000), 127-180. MR 2001j:22024

[KaL] Kazhdan, D. and Lusztig, G., Proof of the Deligne-Langlands conjecture for Hecke algebras, Invent. Math. 87 (1987), 153-215. MR 88d:11121

[KuR] Kudla, S. and Rallis, S., A regularized Siegel-Weil formula: The first term identity, Ann. of Math. 140 (1994), 1-80. MR 95f:11036

[M1] Mœglin C., Normalisation des opérateurs d'entrelacement et réductibilité des induites de cuspidales; le cas des groupes classiques p-adiques, Ann. of Math. 151 (2) (2000), 817-847. MR 2002b:22032

[M2] - Sur la classification des séries discrètes des groupes classiques p-adiques: paramètres de Langlands et exhaustivité, in Journal of the European Mathematical Society (to appear).

[M3] - Représentations quadratiques unipotentes des groupes classiques p-adiques, Duke Math. J. 84 (1996), 267-332. MR 97h:22013

[M4] Non nullité de certains relèvements par série théta, Journal of Lie Theory 7 (1997), 201-229. MR 98m:11044a

[M5] Points de réductibilité pour les induites de cuspidales, Prépublication, Institut de mathématiques de Jussieu (2001).

[MViW] Møglin, C.; Vignéras, M.-F. and Waldspurger, J.-L., Correspondances de Howe sur un corps p-adique, Lecture Notes in Math. 1291, Springer-Verlag, Berlin, 1987. MR 91f:11040

[MW] Møglin, C. and Waldspurger J.-L., Le spectre discret de $G L(n)$, Ann. Sci. École Norm. Sup. 22 (1989), 605-674. MR 91b:22028

[Mu Muić, G., On generic irreducible representations of $S p(n, F)$ and $S O(2 n+1, F)$, Glasnik Mat. 33(53) (1998), 19-31. MR 2000j:22020

[Sh1] Shahidi, F., A proof of Langlands conjecture on Plancherel measures: complementary series for p-adic groups, Ann. of Math. 132 (1990), 273-330. MR 91m:11095

[Sh2] _ On certain L-functions, Amer. J. Math. 103 (1981), 297-356. MR 82i:10030

[Sh3] , Local coefficients and intertwining operators for $G L(n)$, Compositio Math. 48 (1983), 271-295. MR 85a:22027

[Sh4] - Twisted endoscopy and reducibility of induced representations for p-adic groups, Duke Math. J. 66 (1992), 1-41. MR 93b:22034

[Sh5] - Fourier transforms of intertwining operators and Plancherel measures for $G L(n)$, Amer. J. Math. 106 (1984), 67-111. MR 86b:22031 
[Si] Silberger, A., Special representations of reductive p-adic groups are not integrable, Ann. of Math. 111 (1980), 571-587. MR 82k:22015]

[T1] Tadić, M., On regular square integrable representations of p-adic groups, Amer. J. Math. 120 (1) (1998), 159-210. MR 99h:22026

[T2] - On reducibility of parabolic induction, Israel J. Math. 107 (1998), 29-91. MR 2001d:22012

[T3] - Square integrable representations of classical p-adic groups corresponding to segments, Representation Theory 3 (1999), 58-89. MR 2000d:22020

[T4] A family of square integrable representations of classical p-adic groups, preprint (1998).

[T5] Structure arising from induction and Jacquet modules of representations of classical p-adic groups, Journal of Algebra 177 (1) (1995), 1-33. MR 97b:22023

[T6] L Representations of p-adic symplectic groups, Compositio Math. 90 (1994), 123181. MR 95a:22025

[Vo] Vogan, D.A., The local Langlands conjecture, Contemp. Math. 145 (1993), 305-379. MR 94e:22031

[W1] Waldspurger, J.-L., La formule de Plancherel pour les groupes p-adiques, d'après HarishChandra, Journal de l'Institut de Mathématiques de Jussieu (to appear).

[W2] Un exercice sur $G S p(4, F)$ et les représentations de Weil, Bull. Soc. Math. France 115 (1987), 35-69. MR 89a:22033

[Z] Zelevinsky, A. V., Induced representations of reductive p-adic groups II. On irreducible representations of $G L(n)$, Ann. Sci. École Norm. Sup. 13 (1980), 165-210. MR 83g:22012

Institut de Mathématiques de Jussieu, CNRS, F-75251 Paris Cedex 05, France

E-mail address: moeglin@math.jussieu.fr

Department of Mathematics, University of Zagreb, BijeničKa 30, 10000 Zagreb, CroATIA

E-mail address: tadic@math.hr 